\title{
Unidades Hipercentrais em Anéis de Grupo
}

Edson Ryoji Okamoto Iwaki

\author{
DISSERTAÇÃO APRESENTADA \\ $\mathrm{AO}$ \\ INSTITUTO DE MATEMÁTICA E ESTATÍSTICA \\ DA \\ UNIVERSIDADE DE SÃO PAULO \\ PARA \\ OBTENÇÃO DO GRAU \\ $\mathrm{DE}$ \\ MESTRE EM MATEMÁTICA \\ Área de Concentração: Álgebra \\ Orientador: Prof. Dr. Orlando Stanley Juriaans
}

Durante a elaboração deste trabalho o autor recebeu apoio financeiro do CNPq.

-São Paulo, 5 de Julho de 2000- 


\section{Unidades Hipercentrais em Anéis de Grupo}

Este exemplar corresponde à redação final da dissertação devidamente corrigida

defendida por Edson Ryoji Okamoto Iwaki e aprovada pela comissão julgadora.

São Paulo, 5 de Julho de 2000.

Banca examinadora:

- Prof. Dr. Orlando Stanley Juriaans (Orientador) - IME - USP

- Prof. Dr. Francisco César Polcino Milies - IME - USP

- Prof. Dr. Nivaldo Nunes de Medeiros Junior - UFF 
Aos meus pais e irmãos e em memória do meu avô Tsuneyasu Okamoto 


\section{Agradecimentos}

A Deus que me fortaleceu e me animou nos momentos de dificuldade.

Inicialmente gostaria de dizer que somente tenho palavras de elogios e agradecimentos ao meu orientador, Prof. Dr. Orlando Stanley Juriaans, o qual além de nos orientar, sempre nos incentivou a ver mais além. Agradeço a sua paciência e incentivo durante este período.

Aos meus pais, Mituko e Tatuo; irmãos Leonardo, Yurika e Erika e parentes, agradeço por tudo o que fizeram até aqui.

Ao Gláucio, Clézio, Walter, Nelson pela amizade e aos colegas de graduação, mestrado e doutorado, gostaria de expressar os meus agradecimentos.

Ao amigo Antônio Calixto pelos seminários e pelas discussões sempre proveitosas.

Aos professores Profa. Dra. Carmen Silvia Cardassi, Prof. Dr. Roberto Celso Fabrício Costa e Profa. Maria Lúcia Sobral Singer pelas oportunidades de Iniciação Científica. Em particular à saudosa Profa. Dra. Carmen Silvia Cardassi pela sua extrema dedicação e atenção durante o período em que me orientou.

Aos professores do IME por sua dedicação.

Gostaria de expressar também o meu agradecimento a profa. Eliane Hosokawa Imayuki pela leitura do manuscrito.

Ao $\mathrm{CNPq}$ pelo apoio financeiro durante a elaboração desta dissertação. 


\section{Resumo}

Grande parte dos problemas em Anéis de Grupo centraliza-se em torno do estudo do seu grupo de unidades. Torna-se importante então conhecermos a estrutura do grupo de unidades de um anel de grupo $\mathcal{U}_{1}(\mathbb{Z} G)$. No entanto, salvo raras exceções, pouco se conhece acerca da estrutura de $\mathcal{U}_{1}(\mathbb{Z} G)$. Uma das idéias para se conhecer um pouco mais sobre a estrutura do grupo de unidades seria estudarmos a sua série central superior.

No caso em que o grupo $G$ é finito, um resultado de Gruenberg pode ser usado para mostrar que a série central superior de $\mathcal{U}=\mathcal{U}_{1}(\mathbb{Z} G)$ estaciona. Este fato nos possibilita estudarmos o hipercentro de $\mathcal{U}$.

A fim de obter mais informações sobre as unidades hipercentrais de $\mathcal{U}$, nós necessitamos de uma descrição dos subgrupos de torção do hipercentro de $\mathcal{U}$, o qual obtemos através dos resultados de Bovdi sobre os subgrupos normais periódicos de $\mathcal{U}$.

Neste momento, surgem algumas perguntas, as quais procuraremos expor neste trabalho. Entre elas: O limitante superior para a série central superior de $\mathcal{U}$ depende do grupo $G$ ? Como determinar a altura central superior de $\mathcal{U}$ ? Neste momento é interessante salientarmos como a Conjectura do Normalizador nos possibilita obtermos uma estimativa para a altura central de $\mathcal{U}$. Todas estas perguntas são respondidas no capítulo 4, como resultado dos trabalhos de Arora, Hales, Passi que nos garantem que neste caso a altura central de $\mathcal{U}$ é no máximo 2. Embora a demonstração original deste fato, devido a Arora, Hales e Passi, não tenha utilizado a Conjectura do Normalizador, tomamos neste trabalho a idéia de supormos um provável caminho que levasse a este resultado obtendo estimativas para a altura central de $\mathcal{U}$ utilizando a Conjectura do Normalizador e um teorema de Gross. Nosso intuito com isso foi o de conectarmos a resolução do problema em questão com um problema de pesquisa intensa atual na área, ou seja, a Conjectura do Normalizador.

Nesse caso, surge mais uma pergunta: Quais os grupos $G$ tais que $\mathcal{U}$ admite altura central exatamente 0,1 ou 2? Pergunta que é respondida por Arora, Hales e Passi também.

Finalmente, mais um resultado de Arora, Hales e Passi nos mostram uma caracterização do hipercentro de $\mathcal{U}$ que surpreendentemente bate com a estimativa dada pela Conjectura do Normalizador.

É interessante notar aqui o aparecimento da Conjectura do Normalizador tanto para obtermos uma estimativa da altura central de $\mathcal{U}$ como na caracterização do hipercentro de $\mathcal{U}$.

No capítulo 5 apresentamos a generalização dos resultados de Arora, Hales e Passi para o caso em que o grupo $G$ é periódico, cujos resultados se devem basicamente a Y.Li. 
No caso em que o grupo $G$ é periódico, Li mostrou que a altura central de $\mathcal{U}$ é no máximo 2. E introduzindo o conceito de $n$-centro de um grupo, obtém-se uma caracterização do $n$-centro de $\mathcal{U}$ em função dos resultados sobre o hipercentro do grupo de unidades. 


\begin{abstract}
A great deal of problems in Group Rings centralize around the study of its group of units. Hence it becomes important to know the structure of the group of units $\mathcal{U}_{1}(\mathbb{Z} G)$. But with a few exceptions, we do not have much information about its structure. Trying to obtain more information about the structure of $\mathcal{U}_{1}(\mathbb{Z} G)$, we could, for example, study the upper central series of $\mathcal{U}_{1}(\mathbb{Z} G)$.

In case $G$ is finite, a result of Gruenberg implies that $\mathcal{U}_{1}(\mathbb{Z} G)$ has finite central height. This fact allow us to study the hypercenter of $\mathcal{U}$.

In order to obtain more information about the hypercentral units of $\mathcal{U}$ we need a description of the torsion subgroup of the hypercenter of $\mathcal{U}$ which is provided by results of Bovdi on periodic normal subgroups of $\mathcal{U}$.

Gruenberg's result suscites some questions which we will try to answer in this work. Among them: The upper bound for the upper central serie of $\mathcal{U}$ depends on of the group $G$ ? How could we determine the central height of $\mathcal{U}$ ? It is interesting to see how we could obtain an estimative for the central height of $\mathcal{U}$ using the Normalizer Conjecture. All these questions are answered in chapter 4, as a consequence of Arora, Hales and Passi's work which guarantees us that in this case the central height of $\mathcal{U}$ is at most 2. Nevertheless this result of Arora, Hales and Passi doesn't use the Normalizer Conjecture, we suppose here that the Normalizer Conjecture holds and used a result of Gross to obtain estimatives to the central height of $\mathcal{U}$. Our aim was to connect the question discussed ahead with a intensive research problem, the Normalizer Conjecture.

This arises the following question: For which groups does $\mathcal{U}$ have central height exactly 0,1 or 2? This question is also answered by Arora, Hales and Passi.

Finally, another result of Arora, Hales and Passi present us a characterization of the hypercenter of $\mathcal{U}$, which surprisingly satisfies the condition presented in the Normalizer Conjecture.

It is interesting to observe here the appearing of Normalizer Conjecture to obtain an estimative for the central height of $\mathcal{U}$ and to obtain a characterization of the hypercenter of $\mathcal{U}$.

In chapter 5 we present a result of Li which generalizes the result of Arora, Hales and Passi to the case when $G$ is a periodic group. He proves that the central height of $\mathcal{U}$ is also at most 2. Introducing the concept of $n$-center he was able to use the results about the hypercenter of $\mathcal{U}$ to obtain a characterization of the $n$-center of $\mathcal{U}$.
\end{abstract}




\section{Notação}

$|G|$

$o(h)$

$H<G$

$H \triangleleft G$

$A \times B$

$Z(G), \mathcal{Z}(G)$

$N_{G}(H), \mathcal{N}_{G}(H)$

$\operatorname{Aut}(X)$

$\operatorname{Inn}(X)$

anel

Out $(X)$

anel

$C_{G}(H)$

$H^{1}(Y, G)$

$S_{p}(G), \operatorname{Syl}_{p}(G)$

$G L(n, F)$

$S L(n, F)$

$\operatorname{Tri}(n, F)$

$\operatorname{Tri}_{1}(n, F)$ ordem do grupo $G$

ordem do elemento $h$

$H$ subgrupo de $G$

$H$ subgrupo normal de $G$

produto direto dos grupos $A$ e $B$

centro do grupo $G$

normalizador de $H$ em $G$

o conjunto dos automorfismos de $X$, onde $X$ denota um grupo ou anel

o conjunto dos automorfismos internos de $X$, onde $X$ denota um grupo ou

o conjunto dos automorfismos externos de $X$, onde $X$ denota um grupo ou

o centralizador de $H$ em $G$

primeiro grupo de cohomologia de $Y$ com coeficientes em $G$

$p$-subgrupo de Sylow de $G$

grupo linear geral de grau $n$ sobre o corpo $F$

grupo linear especial $=\{g \in G L(n, F) \mid \operatorname{det}(g)=1\}$

grupo triangular $=\left\{\left(g_{i j}\right) \in G L(n, F) \mid g_{i j}=0 \quad\right.$ para $\left.i<j\right\}$

grupo unitriangular $=\left\{\left(g_{i j}\right) \in \operatorname{Tr} i(n, F) \mid g_{i i}=1 \quad\right.$ para $\left.i \neq j\right\}$

$\operatorname{diag}\left(a_{11}, \cdots, a_{n n}\right)$ matriz diagonal de ordem $n$ com elementos $a_{11}, \cdots, a_{n n}$

$D(n, F)$

o grupo diagonal $=\left\{\left(g_{i j}\right) \in G L(n, F) \mid g_{i j}=0 \quad\right.$ para $\left.\quad i \neq j\right\}$

$\pi$

$O_{\pi}(G)$

conjunto de inteiros primos

o único $\pi$-subgrupo normal maximal de $G$, e $\{e\}$ caso $G$ seja um grupo sim-

ples

$O_{\pi, \pi^{\prime}}(G)$

a imagem inversa em $G$ do único $\pi^{\prime}$-subgrupo normal maximal $O_{\pi^{\prime}}\left(G / O_{\pi}(G)\right)$

em $G / O_{\pi}(G)$

$T(G)$

$G^{\prime}$

subgrupo dos elementos de torção do grupo $G$

$[h, k]$

grupo derivado de $G$

$[H, K]$

comutador dos elementos $h$ e $k$

$R(G)$

$\operatorname{tr}(g)$

grupo gerado pelos comutadores $[h, k], h \in H, k \in K$

$\operatorname{car}(K)$

$\bar{F}$

$F^{*}$

$\left[h,{ }_{s} g\right]$

$R_{m}(G)$ intersecção dos subgrupos não-normais do grupo $G$

traço da matriz $g$

característica do corpo $K$

fecho algébrico do corpo $K$

grupo multiplicativo do corpo $K$

$s$-Engel elemento à direita

conjunto dos $m$-Engel elementos à direita 
$R G$

$\operatorname{supp}(\alpha)$

$\epsilon$

$\mathcal{U}(\mathbb{Z} G)$

$\mathcal{U}_{1}(\mathbb{Z} G)$

$\widetilde{Z}(G)$

$[x, y]$

$[\mathbb{Z} G, \mathbb{Z} G]$

$Z(G, n)$

$I_{S}(R)$

anel de grupo $R G$

suporte do elemento $\alpha \in R G$

homomorfismo de aumento do anel de grupo $R G$

grupo de unidades do anel de grupo $\mathbb{Z} G$

grupo de unidades com aumento 1 do anel de grupo $\mathbb{Z} G$

hipercentro do grupo $G$

produto de Lie dos elementos $x$ e $y$

grupo aditivo de $\mathbb{Z} G$ formado pelos produtos de Lie $[x, y], x, y \in \mathbb{Z} G$

$n$-centro do grupo $G$

o conjunto dos elementos de $S$ que são inteiros sobre $R$ 


\section{Sumário}

1 Preliminares $\quad 13$

1.1 Alguns resultados sobre Anéis de Grupo . . . . . . . . . . . . . . . . . . 13

1.2 Alguns resultados sobre Teoria dos Anéis, Módulos e Álgebras . . . . . . . . . . . 19

1.2.1 Tópicos em teoria de representação de grupos . . . . . . . . . . . . . . 20

2 Um resultado de Gruenberg $\quad 21$

2.1 Notação, alguns resultados e definições . . . . . . . . . . . . . . . . . . . . . . 21

2.2 Alguns resultados e definições . . . . . . . . . . . . . . . . . . . . . 22

2.3 O teorema de Gruenberg . . . . . . . . . . . . . . . . . . 26

3 Caracterização dos subgrupos normais de torção de $\mathcal{U}_{1}(\mathbb{Z} G)$ de um grupo $\begin{array}{ll}\text { periódico } G & 29\end{array}$

3.1 Alguns resultados iniciais $\ldots \ldots \ldots \ldots$

3.2 Subgrupos Normais Periódicos de $\mathcal{U}_{1}(\mathbb{Z} G) \ldots \ldots \ldots \ldots$. . . . . . . . 31

3.2.1 Subgrupos Normais Periódicos Não-Abelianos de $\mathcal{U}_{1}(\mathbb{Z} G) \ldots \ldots$

3.2.2 Subgrupos Normais Periódicos Abelianos de $\mathcal{U}_{1}(\mathbb{Z} G) \ldots \ldots . \ldots . \ldots$

3.2.3 Estrutura dos grupos periódicos $G$ tais que $\mathcal{U}_{1}(\mathbb{Z} G)$ contém um subgrupo normal periódico abeliano não-central . . . . . . . . . . . . . . . 45

3.3 Um teorema de Bovdi . . . . . . . . . . . . . . . . . . . . 47

4 A altura central de $\mathcal{U}_{1}(\mathbb{Z} G)$ de um grupo finito 49

$4.1 \quad$ A altura central de $\mathcal{U}_{1}(\mathbb{Z} G)$ é finita $\ldots \ldots \ldots \ldots \ldots \ldots$ 
4.2 Uma estimativa para a altura central de $\mathcal{U}_{1}(\mathbb{Z} G)$ utilizando a Conjectura do Nor-

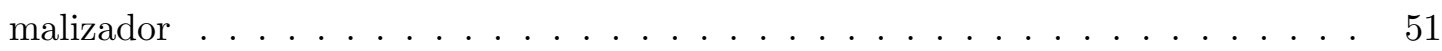

4.2 .1 Notação e algumas definições . . . . . . . . . . . . . . . . . . . . 51

4.2.2 A estimativa para a altura central de $\mathcal{U}_{1}(\mathbb{Z} G) \ldots \ldots \ldots$. . . . . . . 52

4.3 A altura central de $\mathcal{U}_{1}(\mathbb{Z} G)$ é no máximo $2 \ldots \ldots \ldots \ldots \ldots$

4.4 Uma caracterização do segundo centro de $\mathcal{U}_{1}(\mathbb{Z} G) \ldots \ldots \ldots$

4.4.1 O teorema de classificação de $p$-grupos de Blackburn . . . . . . . . . . 60

4.4.2 A Conjectura do Normalizador para grupos G tais que $R(G)$ não é trivial 61

$4.4 .3 \quad \mathrm{O}$ resultado principal . . . . . . . . . . . . . . . . . . . 66

4.5 Caracterização dos grupos finitos $G$ em função da altura central de $\mathcal{U}_{1}(\mathbb{Z} G)$. . $\quad 68$

4.5.1 Alguns Exemplos . . . . . . . . . . . . . . . . . . . . . 69

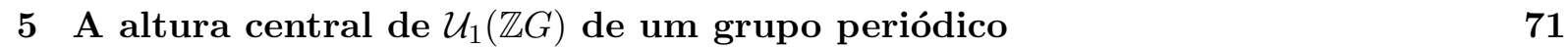

5.1 A altura central de $\mathcal{U}_{1}(\mathbb{Z} G)$ é no máximo $2 \ldots \ldots \ldots \ldots \ldots$

$5.2 \mathrm{O} n$-centro do grupo de unidades de um anel de grupo inteiro $\ldots \ldots \ldots . . .75$

5.2 .1 Alguns resultados básicos e notações . . . . . . . . . . . . . . . . . 75

5.2.2 Uma caracterização do $n$-centro de $\mathcal{U}_{1}(\mathbb{Z} G) \ldots \ldots \ldots . \ldots . \ldots 78$

$\begin{array}{ll}\text { A Um resultado de Borel-Chandra } & 91\end{array}$ 


\section{Capítulo 1}

\section{Preliminares}

Neste capítulo apenas procuramos apresentar alguns resultados que consideramos serem bem conhecidos em Anéis de Grupo e em teoria de Anéis e Grupos, respectivamente. Estes resultados estão indicados nas referências: [30], [31], [28].

\subsection{Alguns resultados sobre Anéis de Grupo}

Definição 1.1. $O$ anel de grupo $R G$ de um grupo $G$ sobre um anel $R$ com identidade, é definido como o conjunto de todas as combinações lineares formais da forma

$$
\alpha=\sum_{g \in G} a_{g} g, a_{g} \in R
$$

tais que:

$$
\operatorname{supp}(\alpha)=\left\{g \mid a_{g} \neq 0\right\},
$$

o suporte de $\alpha$, é finito; com as operações definidas por:

(1) $\sum_{g \in G} a_{g} g+\sum_{g \in G} b_{g} g=\sum_{g \in G}\left(a_{g}+b_{g}\right) g$,

(2) $\left(\sum_{g \in G} a_{g} g\right)\left(\sum_{g \in G} b_{g} g\right)=\sum_{g, h \in G} a_{g} b_{h} g h=\sum_{u \in G} c_{u} u$, onde $c_{u}=\sum_{g h=u} a_{g} b_{h}$.

Podemos definir ainda o produto de um elemento de $R G$ por um escalar $\lambda \in R$ por:

$$
\lambda\left(\sum_{g \in G} a_{g} g\right)=\sum_{g \in G}\left(\lambda a_{g}\right) g .
$$

É fácil de verificar que com a soma e a multiplicação por escalares definida acima, define-se em $R G$ uma estrutura de $R$-módulo. 
Observação 1.2. Observamos que se o anel $R$ for comutativo, então o anel de grupo $R G$, com as operações indicadas acima é também chamada a álgebra de grupo de $G$ sobre $R$.

Definição 1.3. O homomorfismo $\varepsilon: R G \longmapsto R$ dado por:

$$
\varepsilon\left(\sum_{g \in G} a_{g} g\right)=\sum_{g \in G} a_{g}
$$

é chamado o homomorfismo de aumento de $R G$ e seu kernel, denotado por $\triangle(G)$ é chamado o ideal de aumento de $R G$.

Proposição 1.4. O conjunto $\{g-1 \mid g \in G, g \neq 1\}$ é uma base de $\triangle(G)$ sobre $R$.

Da proposição acima podemos concluir que $\triangle(G)$ é um ideal de $R G$ gerado por $\{g-1 \mid g \in G, g \neq 1\}$, isto é, $\triangle(G)=\langle\{g-1 \mid g \in G, g \neq 1\}\rangle$.

Suponha agora que $H \triangleleft G$, então o epimorfismo natural $\omega: G \longmapsto G / H$ pode ser estendido a um epimorfismo $\omega^{*}: R G \longmapsto R(G / H)$ tal que:

$$
\omega^{*}\left(\sum_{g \in G} a_{g}(g)\right)=\sum_{g \in G} a_{g} \omega(g)
$$

Proposição 1.5. Nas condicões acima, temos que: $\operatorname{Ker}\left(\omega^{*}\right)=\triangle_{R}(G, H)=\langle h-1 \mid h \in H\rangle$ como ideal de $R G$.

Proposição 1.6. Seja $R$ um anel comutativo. A aplicação $*: R G \longrightarrow R G$ definida por:

$$
\left(\sum_{g \in G} a_{g} g\right)^{*}=\sum_{g \in G} a_{g} g^{-1}
$$

satisfaz:

(i) $(\alpha+\beta)^{*}=\alpha^{*}+\beta^{*}$;

(ii) $(\alpha \beta)^{*}=\beta^{*} \alpha^{*}$;

(iii) $\alpha^{* *}=\alpha$.

Definição 1.7. Seja $X$ um subconjunto de um anel de grupo $R G$. O anulador à esquerda de $X$ é o conjunto:

$$
A n n_{l}(X)=\{\alpha \in R G \mid \alpha x=0, \forall x \in X\} .
$$

$E$ de maneira análoga, definimos o anulador à direita de $X$ por:

$$
\operatorname{Ann}_{r}(X)=\{\alpha \in R G \mid x \alpha=0, \forall x \in X\}
$$


Definição 1.8. Dado um anel de grupo $R G$ e um subconjunto finito $X$ de um grupo $G$. Denotaremos por $\widehat{X}$ o seguinte elemento de $R G$ :

$$
\widehat{X}=\sum_{x \in X} x
$$

Lema 1.9. Seja $H$ subgrupo de $G$ e seja $R$ um anel. Então: $A n n_{r}(\triangle(G, H)) \neq 0$ se e somente se $H$ é finito. Neste caso, temos que:

$$
A n n_{r}(\triangle(G, H))=\widehat{H} \cdot R G
$$

Além disso, se $H \triangleleft G$ o elemento $\widehat{H}$ é central em $R G$ e temos que:

$$
A n n_{r}(\triangle(G, H))=A n n_{l}(\triangle(G, H))=R G . \widehat{H} .
$$

Lema 1.10. Sejam $R$ um anel com unidade e $H$ um subgrupo de um grupo $G$. Se $|H|$ é invertivel em $R$ então $e_{H}=\frac{1}{|H|} \widehat{H}$ é um idempotente de $R G$. Além disso, se $H \triangleleft G$ então $e_{H}$ é central.

Proposição 1.11. Sejam $R$ um anel com unidade e $H$ um subgrupo normal do grupo $G$. Se $|H|$ é invertível em $R$, considerando $e_{H}=\frac{1}{|H|} \widehat{H}$, nós temos que:

$$
R G=R G e_{H} \oplus R G\left(1-e_{H}\right)
$$

onde

$$
R G e_{H} \cong R(G / H) \quad \text { e } R G\left(1-e_{H}\right)=\triangle(G, H)
$$

Definição 1.12. Um elemento $r$ de um anel $R$ é chamada uma unidade se existe um elemento $s \in R$ tal que $r s=s r=1$. O conjunto de todas as unidades de $R$ forma um grupo $\mathcal{U}(R)$. Denominado o grupo de unidades do anel $R$.

Alguns exemplos de unidades em $\mathbb{Z} G$ :

(1) Os elementos $\pm g, g \in G$ são claramente unidades, sendo os inversos dados por $\pm g^{-1}$. E são chamadas de unidades triviais.

(2) Para $b \in G$ de ordem finita, denotaremos por $\widehat{b}$ a soma:

$$
\widehat{b}=\sum_{1}^{o(b)} b^{i} .
$$

Então $(1-b) \widehat{b}=0$ e para qualquer $a \in G,((1-b) a \widehat{b})^{2}=0$. Portanto, $u_{b, a}=1+(1-b) a \widehat{b}$ possui um inverso $1-(1-b) a \widehat{b}$. As unidades $u_{b, a}$ são chamadas unidades bicíclicas de $\mathbb{Z} G$. 
Seja $u \in \mathcal{U}(\mathbb{Z} G)$. Então $\varepsilon(u)= \pm 1$. Portanto, podemos escrever $\mathcal{U}(\mathbb{Z} G)= \pm \mathcal{U}_{1}(\mathbb{Z} G)$ onde $\mathcal{U}_{1}(\mathbb{Z} G)$ consiste de todas as unidades com aumento 1. Enumeramos a seguir alguns fatos básicos em Anéis de Grupo:

Definição 1.13. Denotaremos por $[\mathbb{Z} G, \mathbb{Z} G]$ o subgrupo aditivo de $\mathbb{Z} G$ gerado por todos os produtos de Lie $[x, y]$, onde $[x, y]=x y-y x$, para todo $x, y \in \mathbb{Z} G$.

Lema 1.14. Seja $u \in \mathcal{U}(\mathbb{Z} G), u=\sum_{g \in G} u(g) g$. Então definimos $\widetilde{u}(g)=\sum_{h \sim g} u(h)$, onde $\sim$ denota a conjugação em $G$. Nessas condições temos que:

$$
[\mathbb{Z} G, \mathbb{Z} G]=\left\{\sum_{x \in G} \alpha(x) x \mid \widetilde{\alpha}(g)=0 \quad \text { para todo } g \in G\right\} .
$$

Teorema 1.15. (Berman) Seja $R$ um domínio de integridade de característica 0 tal que nenhum primo racional é uma unidade de $R$. Então:

$$
\alpha \in R G ; \alpha^{n}=1, \alpha(1) \neq 0 \Rightarrow \alpha=\alpha(1)
$$

Lema 1.16. ([30], corolário 1.7) Qualquer unidade central de ordem finita em $\mathbb{Z} G$ é da forma $\pm t$, onde $t$ é um elemento central em $G$.

Lema 1.17. ([31], proposição 1.3) Se $\gamma \in \mathbb{Z} G$ então $\gamma \gamma^{*}=1 \Leftrightarrow \gamma= \pm g, g \in G$.

Lema 1.18. (Higman) Seja $G$ o grupo dos quatérnions de ordem 8. Então $\mathcal{U}(\mathbb{Z} G)= \pm G$.

Lema 1.19. ([31], corolário 2.3) Seja $G=A \times B$, onde $A$ é um 2-grupo abeliano elementar e $B$ é o grupo dos quatérnions de ordem 8 . Então todas as unidades de $\mathbb{Z} G$ são triviais.

Lema 1.20. Seja $u \in \mathcal{U}$. Então: $u \in \mathcal{N}_{\mathcal{U}}(G) \Leftrightarrow u^{*} u \in \mathcal{Z}=\mathcal{Z}(\mathbb{Z} G)$, onde $\mathcal{N}_{\mathcal{U}}(G)=\{u \in \mathcal{U} \mid$ $\left.u^{-1} G u \subseteq G\right\}$.

Teorema 1.21. ([30], teorema 2.18) Seja G um grupo finito. Então:

$$
T(\mathcal{U}(\mathbb{Z} G))= \pm G \Leftrightarrow G \triangleleft \mathcal{U}(\mathbb{Z} G) \Leftrightarrow(\mathcal{U}(\mathbb{Z} G))^{n} \subseteq \mathcal{Z}(\mathcal{U}(\mathbb{Z} G)) \text { para algum } n \Leftrightarrow
$$

$\Leftrightarrow G$ é Abeliano ou um 2-grupo Hamiltoniano

Teorema 1.22. (Ritter-Sehgal) Seja $G$ um grupo finito. Todas as unidades centrais de $\mathbb{Z} G$ são triviais se e somente se para todo $x \in G$ e todo número natural $j$ relativamente primo com $|G|$, vale que $x^{j} \sim x$ ou $x^{j} \sim x^{-1}$, onde $\sim$ representa a conjugação em $G$.

Lema 1.23. (Krempa) Seja $u \in N_{\mathcal{U}}(G)$. Então $\varphi_{u}^{2}$ é interno, isto é, $u^{2} \in G \mathcal{Z}$, onde $\varphi_{u}$ é o automorfismo de $G$ definido por $\varphi_{u}(g)=u^{-1} g u$, para $g \in G$.

Lema 1.24. (Coleman) Seja $G$ um grupo finito. Então para qualquer $p$-subgrupo $P \leq G$ temos que $N_{\mathcal{U}_{1}(\mathbb{Z} G)}(P)=N_{G}(P) C_{\mathcal{U}_{1}(\mathbb{Z} G)}(P)$, onde $C_{\mathcal{U}_{1}(\mathbb{Z} G)}(P)$ denota o centralizador de $P$ em $\mathcal{U}_{1}(\mathbb{Z} G)$, ou seja, $C_{\mathcal{U}_{1}(\mathbb{Z} G)}(P)=\left\{u \in \mathcal{U} \mid u^{-1} x u=x\right.$, para todo $\left.x \in P\right\}$. 
Proposição 1.25. Seja $R$ um subanel de $\mathbb{Q} G$ contendo $\mathbb{Z} G$. Além disso, suponha que $R$ seja um $\mathbb{Z}$-módulo finitamente gerado. Então $[\mathcal{U}(R), \mathcal{U}(\mathbb{Z} G)]$ é finito.

Proposição 1.26. Seja $G=\left\langle a \mid a^{n}=1\right\rangle$ um grupo cíclico de ordem finita n. Então tem-se que:

$$
\mathbb{Q} G \cong \oplus_{d \mid n} \frac{\mathbb{Q}[X]}{\phi_{d}(X)} \cong \oplus_{d \mid n} \mathbb{Q}\left(\zeta_{d}\right) .
$$

onde $\zeta_{d}$ indica uma raiz primitiva d-ésima da unidade, para cada divisor d de $n$. Neste isomorfismo, o elemento a corresponde ao elemento $\left\{\xi_{d}\right\}_{d \mid n} \in \oplus_{d \mid n} \mathbb{Q}\left(\zeta_{d}\right)$.

Proposição 1.27. Seja A um grupo abeliano finito. Então

$$
\mathbb{Q} A \cong \oplus a_{d} \mathbb{Q}\left(\zeta_{d}\right)
$$

onde $\zeta_{d}$ é uma raiz primitiva d-ésima da unidade e $\varphi(d) a_{d}=n_{d}$, o número de elementos de ordem d em A. Aqui, $a_{d} \mathbb{Q}\left(\zeta_{d}\right)$ denota a soma de $a_{d}$ cópias de $\mathbb{Q}\left(\zeta_{d}\right)$ e $\varphi$ denota a função de Euler.

Lema 1.28. Sejam $K$ corpo, $G, H$ grupos. Então $K G \otimes_{K} K H \cong K[G \times H]$.

Definição 1.29. Diremos que um grupo $G$ é um grupo de Dedekind se todo subgrupo de $G$ é normal em $G$, em particular, se $G$ for um grupo de Dedekind não abeliano então $G$ será denominado um grupo Hamiltoniano.

Proposição 1.30. (Dedekind,Baer)([28], Proposição 5.3.7) Todos os subgrupos de um grupo $G$ são normais se e somente se $G$ é abeliano ou é o produto direto de um grupo quatérnion de ordem 8, um 2-grupo abeliano elementar e um grupo abeliano de ordem ímpar.

Observação 1.31. Em particular, se $G$ for um 2-grupo Hamiltoniano, então $G$ possui a forma $G=Q \times A$, onde $Q$ é o grupo dos quatérnions de ordem 8 e A denota um 2-grupo abeliano elementar.

Se $H$ e $K$ são subconjuntos não vazios de um grupo $G$, então denotaremos por $[H, K]=$ $\langle[h, k] \mid h \in H, k \in K\rangle$, o subgrupo de $G$ gerado pelos comutadores $[h, k]$, onde $[h, k]=$ $h^{-1} k^{-1} h k=h^{-1} h^{k}, h \in H, k \in K$ e $h^{k}=k^{-1} h k$. Quando $H=K=G$, costumamos designar $[G, G]$ por $G^{\prime}$, o grupo derivado de $G$.

Mais genericamente, se $x_{1}, \cdots, x_{n-1}$ são elementos do grupo $G$ um comutador simples de peso $n \geq 2$ é definido recursivamente pela regra:

$$
\left[x_{1}, \cdots, x_{n}\right]=\left[\left[x_{1}, \cdots, x_{n-1}\right], x_{n}\right],
$$

onde convencionamos que $\left[x_{1}\right]=x_{1}$.

A seguir listamos algumas propriedades básicas dos comutadores: 
Proposição 1.32. Sejam $x, y, z$ elementos de um grupo $G$. Então:

(i) $[x, y]=[y, x]^{-1}$;

(ii) $[x y, z]=[x, z]^{y}[y, z]$ e $[x, y z]=[x, z][x, y]^{z}$;

(iii) $\left[x, y^{-1}\right]=\left([x, y]^{y^{-1}}\right)^{-1} e\left[x^{-1}, y\right]=\left([x, y]^{x^{-1}}\right)^{-1}$

(iv) $\left[x, y^{-1}, z\right]^{y}\left[y, z^{-1}, x\right]^{z}\left[z, x^{-1}, y\right]^{x}=1$ (Identidade de Hall-Witt).

Lema 1.33. Seja $G$ um grupo. Se $x$ e y são elementos de $G$, tais que $x$ e y comutam com $[x, y]$ então: $[x, y]^{n}=\left[x^{n}, y\right]=\left[x, y^{n}\right]$ para todo $n \in \mathbb{Z}$.

Demonstração: Suponha que $c=[x, y]=x^{-1} y^{-1} x y$. Então por hipótese temos que $c x=x c$ e $y c=c y$. Segue então que $y\left(x^{-1} y^{-1} x\right)=y\left(x^{-1} y^{-1} x\right) y y^{-1}=y c y^{-1}=c$. Conseqüentemente, $y\left(x^{-1} y^{-1} x\right)=\left(x^{-1} y^{-1} x\right) y$. Logo, $[x, y]^{n}=c^{n}=\left(x^{-1} y^{-1} x\right)^{n} y^{n}=x^{-1}\left(y^{n}\right)^{-1} x y^{n}=\left[x, y^{n}\right]$.

De maneira análoga, mostramos que $c^{n}=\left(x^{-1}\left(y^{-1} x y\right)\right)^{n}=x^{-n}\left(y^{-1} x^{n} y\right)=\left[x^{n}, y\right]$. Concluindo a demonstração.

Definição 1.34. Seja $G$ um grupo qualquer. Uma série ascendente:

$$
(1)=G_{0} \leq G_{1} \leq G_{2} \leq \cdots \leq G_{\alpha} \leq \cdots
$$

num grupo $G$ é chamada central se $G_{\alpha} \triangleleft G$ e cada fator $G_{\alpha+1} / G_{\alpha}$ está contido no centro de $G / G_{\alpha}$ para todo $\alpha$.

Definição 1.35. Seja $G$ um grupo qualquer e $\alpha$ um ordinal. A série central ascendente de $G$ é definida por:

$$
(1)=Z_{0}(G) \leq Z_{1}(G) \leq Z_{2}(G) \leq \cdots \leq Z_{\alpha}(G) \leq \cdots
$$

onde os termos $Z_{\alpha}(G)$ são definidos indutivamente pelas regras:

$$
Z_{0}(G)=1 \quad \text { e } \quad Z_{\alpha+1}(G) / Z_{\alpha}(G)=\mathcal{Z}\left(G / Z_{\alpha}(G)\right)
$$

isto é, $Z_{\alpha+1}(G)$ é a imagem inversa em $G$ de $\mathcal{Z}\left(G / Z_{\alpha}(G)\right)$.

Junto com a condição:

$$
Z_{\lambda}(G)=\bigcup_{\alpha<\lambda} Z_{\alpha}(G)
$$

onde $\lambda$ é um ordinal limite, é denominada de série central superior do grupo $G$.

Observamos que como a cardinalidade de $G$ não pode ser excedida, existe um ordinal $\beta$ tal que $Z_{\beta}(G)=Z_{\beta+1}(G)=\cdots$, e chamaremos de hipercentro ao subgrupo terminal de $G$. 
Lema 1.36. (Mal'cev)([28]) Se o centro de um grupo $G$ é livre de torção então cada fator central superior é livre de torção.

\subsection{Alguns resultados sobre Teoria dos Anéis, Módulos e Álgebras}

Definição 1.37. Seja $R$ anel. Diremos que um $R$-módulo é completamente redutível se ele for a soma direta de módulos simples.

Teorema 1.38. (Artin, Wedderburn) Um anel $R$ é semisimples de comprimento finito se e somente se é da forma:

$$
R \cong M_{n_{1}}\left(D_{1}\right) \oplus \cdots \oplus M_{n_{s}}\left(D_{s}\right)
$$

onde os $D_{i}^{\prime}$ s são anéis de divisão, ou seja, se o anel $R$ é isomorfo a uma soma direta de álgebras de matrizes sobre anéis de divisão.

Proposição 1.39. Seja $R$ um anel semisimples de comprimento finito. Então existe uma família $\left\{e_{1}, \cdots, e_{s}\right\}$ de elementos de $R$ tais que:

(1) $e_{i} \neq 0$ é um idempotente central, isto é, $e_{i} a=a e_{i}, e_{i}^{2}=e_{i}$, para todo $a \in R$ e todo $i$, $1 \leq i \leq s$

(2) Se $i \neq j$, então $e_{i} e_{j}=0$;

(3) $1=e_{1}+\cdots+e_{s}$

(4) $e_{i}$ não pode ser escrito como $e_{i}=e_{i}{ }^{\prime}+e_{i}{ }^{\prime \prime}$, onde $e_{i}{ }^{\prime}, e_{i}{ }^{\prime \prime}$ são idempotentes centrais tais que $e_{i}{ }^{\prime}, e_{i}{ }^{\prime \prime} \neq 0$ e $e_{i}{ }^{\prime} e_{i}^{\prime \prime}=0,1 \leq i \leq s$.

Proposição 1.40. (Maschke) Seja $G$ um grupo finito e seja $K$ um corpo tal que car $(K) \nmid|G|$. Então:

(1) $K G$ é a soma direta de um número finito de ideais bilaterais $\left\{B_{i}\right\}_{1 \leq i \leq r}$, chamadas as componentes simples de KG. Cada $B_{i}$ é um anel simples.

(2) Qualquer ideal bilateral de KG é uma soma direta de alguns dos membros da família $\left\{B_{i}\right\}_{1 \leq i \leq r}$.

(3) Cada componente simples é isomorfa a um anel de matrizes da forma $M_{n_{i}}\left(D_{i}\right)$ onde $D_{i}$ é um anel de divisão contendo uma cópia isomorfa de $K$ em seu centro, e o isomorfismo

$$
K G \cong \oplus_{i=1}^{r} M_{n_{i}}\left(D_{i}\right)
$$

é um isomorfismo de $K$-álgebras. 
Lema 1.41. (Schur) Sejam $R$ um anel, $U, V$ são $R$-módulos irredutíveis. Então qualquer $R$-homomorfismo de $U$ em $V$ é um isomorfismo ou o homomorfismo nulo. $\operatorname{End}_{R}(U)$ é um anel de divisão.

\subsubsection{Tópicos em teoria de representação de grupos}

Definição 1.42. Sejam $G$ um grupo, $F$ corpo e $V$ um $F$-espaço vetorial.

Um homomorfismo $\rho$ de $G$ em $G L(V)$, o grupo de todas as transformações lineares nãosingulares de $V$ é denominada uma representação linear de $G$ sobre $F$, ou simplesmente uma $F$-representação de $G$. Onde aqui assumiremos que $V$ possui dimensão finita $n$. O inteiro $n$ é denominado o grau de $\rho$. Se $\operatorname{Ker}(\rho)=1$, diremos que $\rho$ é fiel.

Suponha que $\left\{v_{1}, \cdots, v_{n}\right\}$ seja uma base (ordenada) de $V$. Então se $g$ pertence a $G$ existe uma matriz $\rho^{*}(g)$ a qual representa a transformação linear $\rho(g)$ com respeito a base. A aplicação $\rho^{*}: G \longmapsto G L(n, F)$ é um homomorfismo o qual será chamado a representação matricial associada com respeito a base dada.

Definição 1.43. Seja $G$ um grupo e suponha que $\rho: G \longmapsto G L(M)$ seja uma F-representação de $G$ de grau n. Então podemos dar uma estrutura de $F G$-módulo a $M$ por meio da seguinte relação:

$$
a\left(\sum_{x \in G} f_{x} x\right)=\sum_{x \in G} f_{x}(a \rho(x)) ;(a \in M) .
$$

A verificação dos axiomas de módulo é simples.

Reciprocamente, se $M$ é qualquer $F G$-módulo a direita com $F$-dimensão finita $n$ então existe uma $F$-representação $\rho: G \longmapsto G L(M)$ de grau $n$ associada dada por:

$$
a \rho(g)=a g \quad(a \in M) .
$$

$O$ que nos mostra que existe uma bijeção entre $F$-representações de $G$ com grau $n e$ FG-módulos à direita de $F$-dimensão $n$.

Definição 1.44. Sejam $G$ um grupo, $F$ corpo e $M$ como na definição anterior. Diremos que uma $F$-representação $\rho$ de $G$ é irredutivel se $M$ não possuir nenhum submódulo não-nulo próprio e ele próprio não ser trivial. Nessas condições diremos que $\rho$ é chamada uma representação irredutivel.

Definição 1.45. Sejam $G$ um grupo, $F$ corpo. Diremos que uma $F$-representação do grupo $G$ será chamada completamente redutivel se ela provém de um FG-módulo completamente redutivel. 


\section{Capítulo 2}

\section{Um resultado de Gruenberg}

Ao iniciarmos o estudo do grupo de unidades de $\mathcal{U}_{1}(\mathbb{Z} G)$ o primeiro caso que consideraremos será o caso em que o grupo $G$ é finito.

Neste caso ao estudarmos a série central superior de $\mathcal{U}_{1}(\mathbb{Z} G)$ veremos que o teorema de Gruenberg desempenha um papel fundamental ao nos garantir que a altura central de $\mathcal{U}_{1}(\mathbb{Z} G)$ é finita, resultado que justifica plenamente os nossos esforços no estudo da série central superior de $\mathcal{U}_{1}(\mathbb{Z} G)$ e em particular, do hipercentro de $\mathcal{U}=\mathcal{U}_{1}(\mathbb{Z} G)$.

Como este teorema desempenhará um papel fundamental no nosso estudo, nosso objetivo principal neste capítulo será o de apresentarmos as idéias principais necessárias a compreensão da sua demonstração, deixando o estudo mais específico da série central superior de $\mathcal{U}_{1}(\mathbb{Z} G)$ para os capítulos 4 e 5 .

Os resultados que serão apresentados a seguir estão relacionados nas referências [18], [19] e [32].

\subsection{Notação, alguns resultados e definições}

Iniciamos nosso estudo, relembrando alguma notação; que é a mesma utilizada nos capítulos anteriores e definida no início da dissertação; e definindo alguns conceitos de teoria de grupos.

Seja $G$ um grupo.

Denotaremos por:

$$
1 \leq Z_{1}(G) \leq Z_{2}(G) \leq \cdots
$$

a série central superior de $G$. 
- $\widetilde{Z}(G)$ o hipercentro do grupo $G$,

- $1_{n}$ a matriz identidade de ordem $n$.

- $\operatorname{Tri}(n, F)$ denotará o grupo triangular $=\left\{\left(g_{i j}\right) \in G L(n, F) \mid g_{i j}=0 \quad\right.$ para $\left.i<j\right\}$,

- $\operatorname{Tr}_{1}(n, F)$ grupo unitriangular $=\left\{\left(g_{i j}\right) \in \operatorname{Tr} i(n, F) \mid g_{i i}=1\right.$ para $\left.i \neq j\right\}$,

- $\operatorname{diag}\left(a_{11}, \cdots, a_{n n}\right)$ matriz diagonal de ordem $n$ com elementos $a_{11}, \cdots, a_{n n}$,

- $D(n, F)$ o grupo diagonal $=\left\{\left(g_{i j}\right) \in G L(n, F) \mid g_{i j}=0 \quad\right.$ para $\left.i \neq j\right\}$.

\subsection{Alguns resultados e definições}

Nesta subseção pretendemos apresentar alguns resultados e definições que serão utilizados para apresentarmos a idéia da demonstração do teorema de Gruenberg que será enunciado mais adiante.

Relembramos que a notação deste capítulo segue a fixada no início da dissertação.

Definição 2.1. Diremos que um grupo abeliano A é um $G$-módulo se existir um homomorfismo de grupos $\phi: G \longmapsto \operatorname{Hom}_{\mathbb{Z}}(A, A)$.

Lema 2.2. Existe uma função a valores inteiros $\mu(n)$ dependendo somente de $n$ tais que:

- Se $n_{1}, \cdots, n_{t}$ são inteiros positivos satisfazendo $n_{1}+n_{2}+\cdots+n_{t}=n$ então

$$
\mu(n) \geq \prod_{i=1}^{t} \mu\left(n_{i}\right) .
$$

- Todo grupo linear completamente redutivel de grau n contém um subgrupo normal abeliano de índice finito no máximo $\mu(n)$.

Definição 2.3. Sejam $G$ um grupo e $V$ um $F G$-módulo. Suponha que $V$ contenha $r$ subespaços não-triviais $V_{1}, \cdots, V_{r}$ tais que $V=V_{1} \oplus \cdots \oplus V_{r}$ e para cada $i$ e cada $g$ em $G, V_{i} g=V_{k}$, onde $k=k(i, g)$. Denote por $k=\sigma(i)$, onde $\sigma \in S_{r}$ e $\pi: g \longmapsto \sigma$ é um homomorfismo de $G$ em $S_{r}$, o grupo simétrico de $r$ elementos. Então o conjunto $\left\{V_{1}, \cdots, V_{r}\right\}$ é chamado um sistema de imprimitividade de $G$ em $V$.

Lema 2.4. Sejam $G$ grupo, $H$ um subgrupo normal de $G$ e $V$ um $F G$-módulo irredutível de $F$-dimensão finita. Então $V$ pode expresso como a soma direta de $F H$-módulos irredutíveis de $F$-dimensão igual. Se $W_{1}, \cdots, W_{r}$ são componentes homogêneas não triviais de $V$ como FH-módulos, então $\left\{W_{1}, \cdots, W_{r}\right\}$ é um sistema de imprimitividade de $G$ em $V$. 
Lema 2.5. (Zassenhaus) Um grupo linear localmente solúvel é solúvel. Em particular, grupos lineares hipercentrais e grupos lineares localmente nilpotentes são solúveis.

Teorema 2.6. (Gruenberg) Sejam $G$ um grupo linear de grau n e $H$ um subgrupo normal solúvel de $G$. Então $H$ contém um subgrupo triangularizável de índice finito o qual é normal em $G$.

Demonstração: Claramente nós podemos supor que $F$ é algebricamente fechado. Seja $H$ subgrupo solúvel de $G L(n, F)$. Existem inteiros $r, n_{1}, \cdots, n_{r}, x$ em $G L(n, F)$ e representações irredutíveis $\rho_{i}$ de $H$ em $G L\left(n_{i}, F\right), i=1, \cdots, r$ satisfazendo

$$
n=n_{1}+\cdots+n_{r} \quad \text { e } \quad g^{x}=\left(\begin{array}{ccc}
\rho_{1}(g) & & 0 \\
& \ddots & \\
& & \rho_{r}(g)
\end{array}\right) \text { para todo } g \text { em } H .
$$

Pelo lema 2.2 temos que $\rho_{i}(H)$ contém um subgrupo normal abeliano $A_{i}$ de índice finito no máximo $\mu\left(n_{i}\right)$. Considere $T=\cap_{i=1}^{r} \rho_{i}^{-1}\left(A_{i}\right)$. $T$ é um subgrupo normal de índice finito no máximo $\mu(n)$ em $H$ pelo lema 2.2. Pelos lemas 2.4 e 1.41, concluímos que $A_{i}$ é um subgrupo diagonalizável de $G L\left(n_{i}, F\right)$. Portanto, $T$ é um subgrupo triangularizável de $G L(n, F)$.

Definição 2.7. Diremos que um elemento $g \in G L(n, F)$ é unipotente se $\left(g-1_{n}\right)^{n}=0$. Por outro lado, diremos que um subgrupo $G$ de $G L(n, F)$ é unipotente se todos os elementos de $G$ são unipotentes.

Definição 2.8. Um subgrupo $G$ de $G L(n, F)$ é dito ser triangularizável sobre $F$ se existe um elemento $x$ de $G L(n, F)$ tal que $G^{x} \subseteq$ Tri $(n, F)$, onde $G^{x}=x^{-1} G x$. Diremos que $G$ é triangularizável se ele for triangularizável sobre $\bar{F}$.

Definição 2.9. Um subgrupo $G$ de $G L(n, F)$ é dito ser unitriangularizável sobre $F$ se existe um elemento $x$ de $G L(n, F)$ tal que $G^{x} \subseteq \operatorname{Tri}_{1}(n, F)$, onde $G^{x}=x^{-1} G x$. Diremos que $G$ é unitriangularizável se ele for unitriangularizável sobre $\bar{F}$.

Definição 2.10. Se $G$ é um subgrupo qualquer de $G L(n, F)$ então a inclusão $G \hookrightarrow G L(n, F)$ é uma representação de $G$ sobre $F$, nesse caso $G$ será chamado irredutível (completamente redutivel) se a correspondente representação for irredutivel (completamente redutivel).

Lema 2.11. (Burnside)([32],lema 1.20) Seja $G$ um subgrupo irredutivel de $G L(n, \bar{F})$ e suponha que $\{\operatorname{tr}(g) \mid g \in G\}$ é um conjunto finito de ordem $m$. Então $G$ é um grupo finito de ordem no máximo $m^{n^{2}}$. Onde $\operatorname{tr}(g)$ denota o traço da matriz $g$ e $\bar{F}$ o fecho algébrico do corpo $F$. 
Corolário 2.12. ([32], corolário 1.21)

Seja $G$ subgrupo unipotente de $G L(n, F)$. Então $G$ é unitriangularizável sobre $F$.

Lema 2.13. Sejam $n, r, d_{1}, \cdots, d_{r}$ inteiros positivos tais que $d_{1}+d_{2}+\cdots+d_{r}=n$. Então $y=\sum_{i=1}^{r} d_{i}\left(n-d_{1}-d_{2}-\cdots-d_{i}\right)$ é menor ou igual a $\frac{1}{2} n(n-1)$ e a igualdade ocorre se $r=n$ e $d_{1}=d_{2}=\cdots=d_{r}=1$.

Demonstração: Se $d_{1}=d_{2}=\cdots=d_{r}=1$ então é imediato que $y=\frac{1}{2} n(n-1)$.

Vamos mostrar agora que $y \leq \frac{1}{2} n(n-1)$.

Se $r=1$ e $d_{1}=n$ temos imediatamente que $y=0 \leq \frac{1}{2} n(n-1)$.

Suponhamos agora que a afirmação é válida para $r=k$ e mostremos que ela é válida para $r=k+1$.

Então pela hipótese de indução podemos supor que $y \leq d_{1}\left(n-d_{1}\right)+\frac{1}{2}\left(n-d_{1}\right)\left(n-d_{1}-1\right)=$ $\frac{1}{2}\left(n^{2}-\left(d_{1}\right)^{2}-n-d_{1}\right)=z$. Calculando o valor máximo de $z$ no intervalo $[1, n]$ obtemos que $z$ admite máximo para $d_{1}=1$. Conseqüentemente, obtemos que $z \leq \frac{1}{2} n(n-1)$.

Lema 2.14. Seja $G$ um grupo, $V$ um $F G$-módulo fiel de dimensão finita sobre $F, W$ um $F G$-submódulo de $V$ e $H$ um subgrupo normal de $G$ estabilizando a série $\{0\} \subseteq W \subseteq V$. Então $H \mathfrak{e} G$ implica que $H \subseteq Z_{t}(G)$, onde $t \leq \operatorname{dim}_{F} W\left(\operatorname{dim}_{F} V-\operatorname{dim}_{F} W\right)$. He $G$ significa que para cada $h$ em $H$ e cada $g$ em $G$ existe um inteiro positivo $s=s(h, g)$ tal que $[h, \underbrace{g, \cdots, g}_{s}]=\left[h,,_{s} g\right]=1$.

Demonstração: Sejam $n=\operatorname{dim}_{F} V$ e $d=\operatorname{dim}_{F} W$. Se $h$ é um elemento de $H$ então

$$
h^{\prime}: a+W \longmapsto a(h-1)
$$

é uma aplicação linear de $V / W$ em $W$, ou seja, $h^{\prime} \in \operatorname{Hom}_{F}(V / W, W)$. Nessas condições a aplicação $\phi: h \longmapsto h^{\prime}$ é um homomorfismo de grupos, do grupo $H$ no grupo aditivo $\operatorname{Hom}_{F}(V / W, W)^{+}$. Se $h^{\prime}$ é a aplicação nula então temos que $a h=a$, para todo $a \mathrm{em} V$. Donde $\phi$ é injetora e em particular, $H$ é abeliano.

$H$ é um $G$-módulo, via conjugação e $H \operatorname{Hom}_{F}(V / W)$ é um $F G$-módulo, onde a ação de $G$ é dada por $\lambda^{g}=g^{-1} \lambda g, \lambda \in \operatorname{Hom}_{F}(V / W, W)$. Agora

$$
\phi\left(h^{g}\right): a+W \longmapsto a\left(h^{g}-1\right) \quad \text { e } \quad(\phi(h))^{g}: a+W \longmapsto a g^{-1}(h-1) g .
$$

Portanto, $\phi$ é um $G$-homomorfismo e conseqüentemente,

$$
\phi\left(\left[h,_{r} g\right]\right)=\phi\left(h^{(g-1) r}\right)=h^{(g-1) r} .
$$


Denotemos por $M$ o $F$-submódulo de $H_{o m}(V / W, W)$ gerado por $\phi(H)$. Como $H \mathfrak{e} G$ e $\operatorname{dim}_{F} M=t$ é finita, cada $g$ em $G$ age de modo unipotente sobre $M$. Pelo corolário 2.12, $G$ age de modo unitriangularizavel relativa a alguma base de $M$. Portanto, se $g_{1}, \cdots, g_{t}$ são $t$ elementos genéricos de $G$ temos que:

$$
\phi\left(\left[h, g_{1}, \cdots, g_{t}\right]\right)=h^{\left(g_{1}-1\right) \cdots\left(g_{t}-1\right)}=0 .
$$

Mas sendo $\phi$ injetora implica que $\left[h, g_{1}, \cdots, g_{t}\right]=1$. O que nos diz que $h \in Z_{t}(G)$.

Apontando que:

$$
t=\operatorname{dim}_{F} M \leq \operatorname{dim}_{F} \operatorname{Hom}_{F}(V / W, W)=d(n-d) .
$$

Concluimos a demonstração do lema.

Corolário 2.15. Sejam $G$ um subgrupo de $G L(n, F)$ e $U$ um subgrupo normal unipotente de $G$. Se $U \mathfrak{e} G$ então $U \subseteq Z_{t}(G)$ onde $t \leq \frac{1}{2} n(n-1)$.

Demonstração: Seja $V$ o espaço gerado pelas linhas de $G$ sobre $F$ considerado como $F G$-módulo a direita.

Pelo corolário 2.12, como $U$ é unipotente, $U$ é unitriangularizável sobre $F$. Logo existe um elemento $w$ em $V$, não-nulo, tal que $w x=w$, para todo $x$ em $U$.

Se $g \in G$ então como $U$ é um subgrupo normal de $G$ temos que $g x g^{-1} \in U$ e conseqüentemente temos que $w g x=w x^{g-1} g=w g$.

Seja $W$ o $F G$-submódulo de $V$ gerado pelo elemento $w$. Nesse caso temos então que $v x=v$, para todo $x \in U$ e $v \in W$.

A seguir, denotemos por $\rho$ a representação do grupo $G$ induzida por $V / W$. Suponha que saibamos que $\rho(U) \subseteq Z_{k}(\rho(G))$, ou seja que, $\left[U,_{k} G\right] \subseteq K=U \cap \operatorname{Ker}(\rho(U))$. Pelo lema 2.14 segue que $K \subseteq Z_{d_{1}\left(n-d_{1}\right)}(G)$, onde $d_{1}=\operatorname{dim}_{F} W$. Conseqüentemente temos que $U \subseteq Z_{t}(G)$ onde $t=k+d_{1}\left(n-d_{1}\right)$. Por indução em $n$ segue que existem inteiros $r, d_{1}, \cdots, d_{r}$ tais que $d_{1}+d_{2}+\cdots+d_{r}=n$ e $U \subseteq Z_{t}(G)$, onde $t=\sum_{i=1}^{r} d_{i}\left(n-d_{1}-d_{2}-\cdots-d_{i}\right)$.

Utilizando o lema 2.13 concluimos a demonstração do corolário.

Teorema 2.16. (Samuel)([29],teo. 1) Seja A uma $\mathbb{Z}$-álgebra comutativa finitamente gerada. Então o grupo de unidades de $A$ é finitamente gerado.

Definição 2.17. Diremos que $K$, o subanel do corpo $F$ gerado por $1_{F}$ será denominado o anel primo do corpo $F$. 
Lema 2.18. (Wehrfritz) Sejam $R$ um subanel finitamente gerado sobre o anel primo do corpo $F$ e $T$ um subgrupo triangularizável de $G L(n, F)$ contido em $G L(n, R)$. Se $U$ é um subgrupo maximal unipotente de $T$ então $T / U$ é um grupo abeliano finitamente gerado.

Demonstração: Podemos supor que o corpo $F$ é algebricamente fechado. Então existe $x$ em $G L(n, F)$ tal que $T^{x} \subseteq \operatorname{Tr} i(n, F)$, onde $\operatorname{Tr} i(n, F)$ denota o conjunto das matrizes triangulares superiores de ordem $n$ com entradas em $F$.

Sejam $x=\left(x_{i j}\right)$ e $x^{-1}=\left(y_{i j}\right)$ e consideremos $S=R\left[x_{i j}, y_{i j} \mid i, j=1, \cdots, n\right] \subseteq F$. Pelo teorema 2.16 concluímos que o grupo de unidades de $S$ é finitamente gerado, visto que $S$ é um domínio de integridade finitamente gerado.

Considere a seguir a aplicação $\theta: T^{x} \longmapsto D(n, S)$, definida por $\theta\left(a_{i j}\right)=\operatorname{diag}\left(a_{11}, \cdots, a_{n n}\right)$, onde $D(n, S)$ denota o conjunto das matrizes diagonais com entradas em $S$ de ordem $n$ e $\operatorname{diag}\left(a_{11}, \cdots, a_{n n}\right)$ representa a matriz diagonal com elementos $a_{11}, \cdots, a_{n n}$.

Podemos observar que $\theta$ é um homomorfismo e que $\operatorname{Ker}(\theta)$ é igual a $U^{x}$.

Como $D(n, S)$ é isomorfo ao produto direto de $n$ cópias do grupo de unidades de $S$ podemos concluir que $D(n, S)$ é finitamente gerado, pois como vimos anteriormente o grupo de unidades de $S$ é finitamente gerado.

Como $D(n, S) \cong T / U \cong T^{x} / U^{x}$, segue que o grupo $T / U$ é abeliano finitamente gerado.

\subsection{O teorema de Gruenberg}

Finalmente nesta subseção apresentamos o resultado principal do capítulo. Utilizando os resultados anteriores apresentamos a demonstração do teorema de Gruenberg.

Teorema 2.19. (Gruenberg)([19], teorema 4)

Se $G$ é um grupo linear finitamente gerado sobre um anel comutativo $R$ então $G$ possui altura central finita.

Demonstração: Empregando a notação que fixamos na subseção anterior, seja $\widetilde{Z}(G)$ o hipercentro de $G$.

Pelo lema 2.5 concluímos que $\widetilde{Z}(G)$ é um subgrupo solúvel de $G$. Como $\widetilde{Z}(G)$ também é normal em $G$, nas hipóteses do teorema 2.6 concluímos que $\widetilde{Z}(G)$ contém um subgrupo triangularizável $T$ de índice finito, normal em $G$. 
Consideremos agora $U$ um subgrupo maximal unipotente de $T$. Então pelo corolário 2.15 concluiríamos que $U \subseteq Z_{\frac{1}{2} n(n-1)}(G)$.

Pela escolha de $T$ e o lema 2.18 temos que $\widetilde{Z}(G) / U$ é uma extensão finita de um grupo abeliano finitamente gerado. Segue daí que o quociente $\widetilde{Z}(G) / U$ satisfaz a condição maximal para subgrupos e portanto, podemos concluir que $G$ possui altura central finita. 


\section{Capítulo 3}

\section{Caracterização dos subgrupos normais de torção de $\mathcal{U}_{1}(\mathbb{Z} G)$ de um grupo periódico $G$}

Iniciamos este capítulo estudando a estrutura dos subgrupos normais periódicos de $\mathcal{U}=$ $\mathcal{U}_{1}(\mathbb{Z} G)$ quando $G$ é um grupo periódico. O objetivo é procurarmos mais informações sobre a estrutura de $\mathcal{U}$.

Nosso estudo dos subgrupos normais de $\mathcal{U}_{1}(\mathbb{Z} G)$ se justifica ao observarmos que o conjunto dos elementos de torção do hipercentro de $\mathcal{U}$ forma um subgrupo normal periódico de $\mathcal{U}$. Esse resultado será mostrado na última seção do capítulo.

Com essa motivação passamos a procurar uma caracterização dos subgrupos normais periódicos de $\mathcal{U}$.

Apresentamos o objetivo principal deste capítulo que será o de demonstrarmos o teorema de Bovdi, que caracteriza os grupos $G$ em função das informações sobre o subgrupo de torção do hipercentro de $\mathcal{U}$.

\subsection{Alguns resultados iniciais}

Começamos então o nosso estudo com o seguinte:

Lema 3.1. [Zassenhaus]([31], Teorema 2.1) Suponha que $R$ é um domínio de integridade de característica 0 no qual nenhum primo racional é invertivel. Se $\mathcal{U}_{1}(R G)$ possui um elemento $\sum_{g \in G} u(g) g$ de ordem $p^{\alpha}, p$ primo, então $G$ possui um elemento $g_{0}$ de ordem $p^{\alpha}$ com $u\left(g_{0}\right) \neq 0$. 
Teorema 3.2. (Bovdi, [30]) Seja I um domínio de integridade de característica 0 tal que nenhum primo racional seja uma unidade de I. Então todo subgrupo normal periódico $N$ de $\mathcal{U}_{1}(I G)$ está contido em $G$. Todo subgrupo de $N$ é normal em $G$. Além disso, $N$ é abeliano ou um 2-grupo Hamiltoniano.

Demonstração: Seja $\alpha=\sum_{g \in G} \alpha(g) g \in N \triangleleft \mathcal{U}(I G)$. Desejamos mostrar que $\alpha \in G$. Sem perda de generalidade, podemos supor que $\alpha$ possui ordem $p^{m}$. De fato, se $o(\alpha)=n=$ $\prod_{i=1}^{k} p_{i}{ }^{r_{i}}, p_{j}$ primos distintos, $\alpha$ pode expresso como $\alpha=\alpha_{1} \alpha_{2} \cdots \alpha_{k}, o\left(\alpha_{i}\right)=p_{i}^{r_{i}}$. Nesse caso, $\langle\alpha\rangle=\prod_{i}\left\langle\alpha_{i}\right\rangle$. Se mostrarmos que $\alpha_{i} \in G, \forall i$ então $\alpha \in G$.

Pelo lema 3.1;

$$
\alpha=\sum_{g \in G} \alpha(g) g \in I G, o(\alpha)=p^{m} \quad \text { e } \quad \alpha\left(g_{0}\right) \neq 0
$$

Então considere $\beta=\alpha g_{0}{ }^{-1}=\sum_{g \in G} \beta(g) g$ o qual satisfaz $\beta(1) \neq 0$ e

$$
\begin{aligned}
\beta^{s} & =\left(\alpha g_{0}{ }^{-1}\right)^{s}= \\
& =\alpha g_{0}{ }^{-1} \alpha g_{0}{ }^{-1} \cdots \alpha g_{0}{ }^{-1}= \\
& =\alpha \alpha^{g_{0}} \alpha^{g_{0}{ }^{2}} \cdots \alpha g_{0}{ }^{s-1} g_{0}{ }^{-s}
\end{aligned}
$$

Tomando $s=p^{m}$ nós obtemos que $\beta^{s} \in N$.

Como $N$ é subgrupo normal de torção, segue que $\beta$ é um elemento de ordem finita do anel de grupo $I G$.

Segue, pelo lema 1.15, que $\beta=1$ e portanto, $\alpha=g_{0} \in G$.

Mostraremos a seguir que todo subgrupo cíclico $\langle h\rangle$ de ordem $n$ de $N$ é normal em $G$.

Para $g \in G$ consideremos: $\mu=\widehat{h} g(1-h)$.

Então: $\mu^{2}=0$ e $(1+\mu)(1-\mu)=1$. Portanto,

$$
\begin{aligned}
(1+\mu)^{-1} h(1+\mu) & =h-\mu h+h \mu=\mu h \mu \\
& =h-\mu h+\mu \\
& =h+\widehat{h} g(1-h)^{2}
\end{aligned}
$$

é um elemento de $N$. Isso implica uma relação do tipo:

$h^{s} g=h^{t} g h$ para algum $s \geq 0$ e $t \leq n-1$.

Portanto, $g h g^{-1} \in\langle h\rangle$ e $\langle h\rangle$ é normal em $G$. Logo, pela proposição 1.30, $N$ é abeliano ou Hamiltoniano. 
Suponha que $N$ é Hamiltoniano. Então afirmamos que:

$$
\gamma=\sum_{g \in G} \gamma(g) g \in \mathcal{U}_{1}(\mathbb{Z} N), \gamma^{m}=1, \text { para algum } m \in \mathbb{Z} \Rightarrow \gamma=g \in N .
$$

Suponha que $\gamma\left(g_{1}\right) \neq 0$. Então $N$ é normal em $\mathcal{U}_{1}(\mathbb{Z} N), \gamma g_{1}{ }^{-1}$ é um elemento de torção de $\mathcal{U}_{1}(\mathbb{Z} N)$ e é portanto, 1 pelo teorema 1.15. Portanto, $\gamma=g_{1}$. Provamos que todas as unidades de torção de $\mathbb{Z} N$ são triviais. Segue do teorema 1.21 que $N$ é um 2-grupo, completando a demonstração.

Lema 3.3. (Bovdi,[15], lema 3) Seja G um grupo periódico. Então são equivalentes:

(1) $G$ é um subgrupo normal de $\mathcal{U}_{1}(\mathbb{Z} G)$;

(2) $G$ é abeliano ou um 2-grupo Hamiltoniano

Demonstração: $(1) \Rightarrow(2)$ :

Segue do teorema 3.2;

$(2) \Rightarrow(1):$

Se $G$ é abeliano então $G \triangleleft \mathcal{U}_{1}(\mathbb{Z} G)$.

Suponhamos agora que $G$ seja um 2-grupo Hamiltoniano.

Logo pelo pelo lema 1.19, todas as unidades de $\mathcal{U}_{1}(\mathbb{Z} G)$ são triviais. Concluindo a demonstração do lema.

\subsection{Subgrupos Normais Periódicos de $\mathcal{U}_{1}(\mathbb{Z} G)$}

Ao tomarmos em consideração o teorema 3.2, surgem algumas perguntas que motivaram o estudo inicial dos subgrupos normais periódicos de $\mathcal{U}$. Entre elas citamos: Como a existência de um subgrupo normal periódico $H$ de $\mathcal{U}_{1}(\mathbb{Z} G)$ de um grupo periódico $G$ se reflete na estrutura do grupo $G$ e do anel $\mathbb{Z}$ ? Qual o posicionamento desse subgrupo normal no grupo $G$ ?

Ao procurar responder essas perguntas, Bovdi notou que se esse subgrupo normal periódico $H$ for não abeliano então os elementos de torção de $H$ estão contidos no conjunto dos elementos de torção do grupo $G$. Enquanto que se $H$ fosse abeliano então a estrutura de $H$ dependeria do posicionamento do grupo dos quatérnions no grupo $G$. 
Tendo em vista essas considerações, provamos o seguinte teorema sobre os subgrupos normais periódicos de $\mathcal{U}_{1}(\mathbb{Z} G)$.

Definição 3.4. Diremos que um group basis do anel de grupo $\mathbb{Z} G$ é um subgrupo $H$ de $\mathcal{U}_{1}(\mathbb{Z} G)$ tal que os elementos de $H$ são linearmente independentes sobre $\mathbb{Z} e \mathbb{Z} G=\mathbb{Z} H$.

Teorema 3.5. (Bovdi,[15], teorema 2) Sejam G um grupo qualquer e $N$ um subgrupo normal periódico de $\mathcal{U}_{1}(\mathbb{Z} G)$. Então todo subgrupo de $N$ é um subgrupo normal de $\mathcal{U}_{1}(\mathbb{Z} G)$. Além disso, $N$ é abeliano ou um 2-grupo Hamiltoniano.

Demonstração: Podemos considerar $N$ como subgrupo normal de $\mathcal{U}_{1}(\mathbb{Z} N)$ e pelo teorema 3.2 segue que $N$ é abeliano ou um 2-grupo Hamiltoniano.

Mostraremos agora que todo subgrupo cíclico $\langle h\rangle$ de ordem $m$ em $N$ é um subgrupo normal de $\mathcal{U}_{1}(\mathbb{Z} G)$.

Suponha que $h^{\prime}=x h x^{-1} \notin\langle h\rangle$.

Nesse caso $y=1+n \widehat{h} x(1-h)$ é uma unidade não-trivial do grupo de unidades $\mathcal{U}_{1}(\mathbb{Z} G)$ para todo inteiro não-nulo $n$ e temos que

$$
y^{-1} h y=(1-n \widehat{h} x(1-h)) h(1+n \widehat{h} x(1-h))=h+n \widehat{h} x(1-h)^{2}
$$

é um elemento de $N$. Escolhido o inteiro $n$, sempre é possível obtermos os coeficientes de $h$ diferentes de zero na expansão de $y^{-1} h y \in \mathbb{Z} N$ em termos do group basis $N$, donde concluímos que $y^{-1} h y=h$ e $\widehat{h} x\left(1-h^{\prime}\right)^{2}=0$, onde $h^{\prime}$ é um elemento de $N$. Segue imediatamente que $x h x^{-1}=h^{\prime} \in\langle h\rangle$. Contradição.

\subsubsection{Subgrupos Normais Periódicos Não-Abelianos de $\mathcal{U}_{1}(\mathbb{Z} G)$}

Passamos a obter mais informações sobre os grupos periódicos $G$ para os quais o grupo de unidades $\mathcal{U}_{1}(\mathbb{Z} G)$ possui um subgrupo normal periódico não-abeliano.

Proposição 3.6. Sejam $G$ um grupo arbitrário e $H$ um subgrupo normal periódico de $\mathcal{U}_{1}(\mathbb{Z} G)$. Se $g$ é um elemento de torção de $G$ com a propriedade que $\langle g\rangle \cap H=\{1\}$ então g pertence ao centralizador de $H$ em $G$.

Demonstração: Suponha que $g$ seja um elemento de ordem $m$ e que $\langle g\rangle \cap H=\{1\}$, e sejam $B=\langle g, h\rangle, h \in H$, e $1, b_{1}, \cdots, b_{s}$ os elementos de $B$ e suponhamos que:

$$
\begin{aligned}
& \widehat{g} h(1-g) h-h \widehat{g} h(1-g)=\gamma_{0} .1+\gamma_{1} b_{1}+\cdots+\gamma_{s} b_{s} \text { e que: } \\
& -\widehat{g} h(1-g) h \widehat{g} h(1-g)=\beta_{0} .1+\beta_{1} b_{1}+\cdots+\beta_{s} b_{s} .
\end{aligned}
$$


Então para todo inteiro $k$ o elemento $y=1+k \widehat{g} h(1-g) \in \mathcal{U}_{1}(\mathbb{Z} G)$ e $y h y^{-1}=h+k\left[\left(\gamma_{0}+\right.\right.$ $\left.\left.k \beta_{0}\right) .1+\cdots+\left(\gamma_{s}+k \beta_{s}\right) b_{s}\right]$ pertence a $\langle h\rangle$ (o qual é um subgrupo de torção de $G$ ), mas isso somente ocorre se $\gamma_{i}=\beta_{i}=0$, para todo $i, 0 \leq i \leq s$. Portanto, se $h g=g h^{p}$, para algum $p$ temos então que:

$$
\widehat{g} h(1-g) h-h \widehat{g} h(1-g)=\widehat{g}\left(h-h^{p}\right) h-h \widehat{g}\left(h-h^{p}\right)=0 .
$$

Logo, se $h \neq h^{p}$, então concluímos que vale a relação $g h^{2}=h g h$, o qual mostra que $g$ e $h$ comutam. Concluindo a demonstração do lema.

Lema 3.7. (Bovdi, [15], lema 2) Seja G um grupo finito que contém dois elementos g, $h$ tais que $G$ é da forma $G=\langle g\rangle\langle h\rangle,\langle g\rangle \cap\langle h\rangle=\{1\}$. Se $\langle h\rangle \triangleleft \mathcal{U}_{1}(\mathbb{Z} G)$ então $G$ é um grupo abeliano.

Demonstração: Segue da demonstração da proposição 3.6.

Teorema 3.8. (Bovdi,[15], teorema 3) Se um 2-grupo Hamiltoniano $H$ de $G$ é um subgrupo normal de $\mathcal{U}_{1}(\mathbb{Z} G)$ então o conjunto dos elementos de ordem finita em $G$ formam um 2-grupo Hamiltoniano $S$ e todo subgrupo de $S$ é um subgrupo normal de $G$.

Demonstração: Desejamos mostrar que todo subgrupo cíclico finito de $G$ é normal em $G$.

Afirmamos inicialmente que $G$ não possui elementos $g$ de ordem finita $n(n \neq 2)$ tais que $\langle g\rangle \cap H=1$.

Suponha que exista algum elemento $g \in G$ de ordem finita $n(n \neq 2)$ tal que $\langle g\rangle \cap H=1$. Segue do lema 3.7 que $g \in C_{G}(H)$ e o grupo $P=\langle g\rangle \times H$ é um subgrupo normal de $\mathcal{U}_{1}(\mathbb{Z} P)$ pois $g$ é um elemento central de $P$. Pelo lema 3.3, $P$ é um 2-grupo Hamiltoniano o que é uma contradição, pois os elementos centrais de um 2-grupo Hamiltoniano possuem ordem 2. Portanto, qualquer elemento $g$ de ordem finita possui ordem $2^{s}$ e se $s>1$ então $\langle g\rangle \cap H \neq 1$. Além disso, se $s \geq 3$ então $g^{2} \in C_{G}(H)$.

Se $g \notin C_{G}(H)$ então como todo subgrupo cíclico de $H$ é normal em $G$ (pelo teorema 3.5 ), $\varphi_{g}$ induz um automorfismo de ordem dois em $H$, onde $\varphi_{g}$ denota o automorfismo interno de $G$ induzido pelo elemento $g$. Então o subgrupo $P=H\left\langle g^{2}\right\rangle$ é um subgrupo normal de $\mathcal{U}_{1}(\mathbb{Z} P)$. Contradição com o lema 3.3, pois nesse caso $P$ seria um 2-grupo Hamiltoniano, $g^{2}$ seria um elemento central em $P$ de ordem maior ou igual a 4 . Portanto, podemos concluir que $s \leq 2$.

Pela proposição 1.30, podemos representar $H$ como $H=Q \times W$, onde $Q$ representa o grupo dos quatérnions de ordem 8 e $W$ é um 2-grupo abeliano elementar.

Suponhamos que $s=2$ e $g \in C_{G}(H)$ então $P=H\langle g\rangle$ seria um subgrupo normal de $\mathcal{U}_{1}(\mathbb{Z} P)$. Pelo lema 3.3, $P$ seria um 2-grupo Hamiltoniano, $g$ seria um elemento central em $P$ de ordem 


\section{Caracterização dos subgrupos normais de torção de $\mathcal{U}_{1}(\mathbb{Z} G)$ de um grupo periódico}

4. Contradição. Logo, $g \notin C_{G}(H)$. Além disso, com base no teorema 3.5 os seguintes casos são possíveis:

(1) $g^{-1} a g=a, g^{-1} b g=a^{2} b$;

(2) $g^{-1} a g=a^{3}, g^{-1} b g=b$;

(3) $g^{-1} a g=a^{3}, g^{-1} b g=a^{2} b$.

Afirmamos agora que o subgrupo $\langle g\rangle H$ de $G$, onde $g$ é um elemento de ordem $2^{s}(s=1$ ou 2 ), pode ser representado como o produto direto do subgrupo $H$ e um subgrupo cíclico $\langle v\rangle$ de ordem 2 .

Suponha que $g$ seja um elemento de ordem 4 e consideremos o caso (1) acima. Tomando $v=a g$, temos que $b v=v b$ e $\varphi_{v}$ induz o automorfismo identidade em $H$.

O grupo $P=\langle v\rangle H$ é um subgrupo normal de $\mathcal{U}_{1}(\mathbb{Z} P)$ e pelo lema $3.3, v$ possui ordem 2 , pois é um elemento central em $P$.

Os casos 2 e 3 são tratados de maneira análoga, com a única ressalva que tomamos $v=a b g$ no caso 3 .

Se $g$ é um elemento de ordem 2 então: $\langle g\rangle H=\langle g\rangle \times H$

Afirmamos que o grupo cíclico $\langle v\rangle$ é um subgrupo normal de $G$.

De fato, se $[v, g] \neq 1, g \in G$ então sempre podemos escolher um elemento $g \in G$ tal que $\langle g\rangle \cap H=1$; caso contrário, $g$ é um elemento de ordem finita e pelo que vimos anteriormente, $g$ pode ser representado na forma $g=h v^{\prime}, \operatorname{com} h \in H,\left\langle v^{\prime}\right\rangle \cap H=1$ e $[v, g]=\left[v, v^{\prime}\right]$.

Então o elemento $y=(1-v) g(1+v)$ é um elemento nilpotente não-nulo e $y^{2}=0$. Pelo teorema $3.5, \varphi_{g}$ induz o automorfismo identidade no subgrupo $W$, enquanto a sua ação no subgrupo $Q$ é determinada de uma das seguintes maneiras:

(1) $g^{-1} a g=a, g^{-1} b g=b$;

(2) $g^{-1} a g=a^{3}, g^{-1} b g=b$;

(3) $g^{-1} a g=a, g^{-1} b g=b^{3}$;

(4) $g^{-1} a g=a^{3}, g^{-1} b g=b^{3}$.

Então, para os casos correspondentes, construímos elementos invertíveis:

(1) $(1-b y) a(1+b y)=a+\left(a b-a^{3} b\right) y$,

(2) $(1-y) a(1+y)=a+\left(a-a^{3}\right) y$, 
(3) - (4) $(1-y) b(1+y)=b+\left(b-b^{3}\right) y$,

os quais pertencem a $H$. Mas esses elementos somente pertencem a $H$ se $y=0$, pois $g \notin H$ e $v \notin H$. Portanto, pelo teorema 3.5, nós concluímos que todo subgrupo cíclico finito de $G$ é um subgrupo normal de $G$. Completando a demonstração.

\subsubsection{Subgrupos Normais Periódicos Abelianos de $\mathcal{U}_{1}(\mathbb{Z} G)$}

Em toda esta subseção, com exceção do lema $3.15, G$ é um grupo não abeliano tal que $\mathcal{U}_{1}(\mathbb{Z} G)$ contém um subgrupo normal periódico abeliano.

Suponha que $H$ seja um subgrupo normal periódico de $\mathcal{U}_{1}(\mathbb{Z} G)$. Passamos então a estudar os seguintes subcasos:

(1) Suponha que o grupo $G$ não possua um subgrupo quatérnion $Q$ tal que $Q \cap H$ seja um grupo cíclico de ordem 4.

Então temos o seguinte teorema:

Teorema 3.9. (Bovdi,[15], teorema 6) Seja $H$ um subgrupo abeliano periódico de $G$, o qual é um subgrupo normal de $\mathcal{U}_{1}(\mathbb{Z} G)$. Se $G$ não contém um grupo quatérnion $Q$ tal que $Q \cap H$ é um grupo cíclico de ordem 4, então o centralizador de $H$ contém todos os elementos de ordem finita de $G$.

Demonstração: Suponha que o centralizador do subgrupo $H$ não contenha algum elemento de ordem finita em $G$. Então existem elementos $h \in H$ e $g_{1} \in G$, onde $h$ é um $p$-elemento e $g_{1}$ é um $q$-elemento,tais que o subgrupo $A_{1}=\left\langle h, g_{1}\right\rangle$ não é abeliano. Pelo lema 3.7 concluímos que $p=q$ e $\langle h\rangle \cap\left\langle g_{1}\right\rangle \neq 1$, caso contrário, $A_{1}$ seria abeliano, pois o subgrupo $\langle h\rangle$ é normal em $\mathcal{U}_{1}\left(\mathbb{Z} A_{1}\right)$. Existe um subgrupo não-abeliano $A$ de $A_{1}, A$ gerado pelos elementos $h$ e $g \in\left\langle g_{1}\right\rangle$, tais que $g$ induz um automorfismo de ordem $p$ no subgrupo $\langle h\rangle$. Concluímos então que $g^{p}$ é um elemento central em $A$ e o subgrupo $B=\langle h\rangle\left\langle g^{p}\right\rangle$ é normal em $\mathcal{U}_{1}(\mathbb{Z} A)$.

Sejam $p^{m}$ e $p^{m_{1}}$ as ordens, respectivamente, dos elementos $h$ e $g . A$ é dado pelas seguintes relações:

$$
h^{p^{m}}=1 ; g^{p^{n}}=h^{t} ; g h g^{-1}=h^{r} ; g^{p} h=h g^{p} \quad \text { para algum } r .
$$

Passemos a análise dos casos possíveis:

(a) $p^{m_{1}}>p^{m}$.

Nesse caso podemos representar $B$ como o produto direto do grupo cíclico $\left\langle g^{p}\right\rangle$ e um grupo cíclico $\left\langle h^{\prime}\right\rangle$. A é a extensão de $\left\langle h^{\prime}\right\rangle$ por $\langle g\rangle$, onde $\left\langle h^{\prime}\right\rangle$ é um subgrupo normal 
de $\mathcal{U}_{1}(\mathbb{Z} A)$ pelo teorema 3.5.Contradição com o lema 3.7. Portanto, os elementos $g$ e $h$ comutam.

(b) $p^{m} \geq p^{m_{1}}, p$ ímpar.

Então: $h=g^{p} h g^{-p}=h^{r^{p}}$ e $r^{p} \equiv 1\left(\bmod p^{m}\right)$. Destas relações segue que $r=1+k p^{m_{1}}$ $\left(\bmod p^{m}\right)$, onde $k \not \equiv 0(\bmod p)$, pois $r \not \equiv 1\left(\bmod p^{m}\right)$. Portanto, existe $i$ tal que $i k \equiv 1(\bmod p)$. Seja $g_{1}=g^{i}$, então nessas condições temos que $g_{1} h g_{1}^{-1}=h^{1+p^{m-1}}$. Donde:

$$
\left(h^{j} g_{1}\right)^{p}=h^{j} h^{j\left(1+p^{m-1}\right)} \cdots h^{j\left(1+p^{m-1}\right)^{p-1}} g_{1}^{p}=h^{j p} g_{1}^{p}
$$

Como $g_{1}^{p}$ comuta com $h$, temos que: $\left(h^{j} g_{1}\right)^{p^{n}}=h^{j p^{n}} g_{1}^{p^{n}}$. Além disso, da relação $1=g_{1}^{p^{m}}=\left(g^{p^{n}}\right)^{i p^{m-n}}=h^{i t p^{m-n}}$, nós temos $t=p^{n} \alpha$ e pelas relações precedentes que: $\left(h^{-i \alpha} g_{1}\right)^{p^{n}}=h^{-i \alpha p^{n}} g_{1}^{p^{n}}=h^{-i t} g^{i p^{n}}=1$.

Então a intersecção dos grupos cíclicos $\langle h\rangle$ e $\left\langle h^{-i \alpha} g_{1}\right\rangle$ é igual a 1 e $\langle h\rangle$ é um subgrupo normal de $\mathcal{U}_{1}(\mathbb{Z} A)$. Contradição com o lema 3.7, pois os elementos $h$ e $h^{-i \alpha} g_{1}$ não comutam.

Portanto, se $p \neq 2$ então o centralizador do $p$-subgrupo de Sylow de $H$ contém todos os elementos de ordem finita em $G$.

(c) $2^{m} \geq 2^{m_{1}}$.

Da relação $h g^{2}=g^{2} h$ segue que $r^{2} \equiv 1\left(\bmod 2^{m}\right)$ e $r \not \equiv 1\left(\bmod 2^{m}\right)$. Temos então três possibilidades para $r: r \equiv-1\left(\bmod 2^{m}\right), r \equiv 1+2^{m-1}\left(\bmod 2^{m}\right), r \equiv$ $-1+2^{m-1}\left(\bmod 2^{m}\right)$. Além disso, da igualdade $g h^{t} g^{-1}=h^{t r}=h^{t}$ temos que $t r \equiv t$ $\left(\bmod 2^{m}\right)$.

Consideremos os subcasos:

(i) $r \equiv-1\left(\bmod 2^{m}\right)$ e $A$ não é um grupo quatérnion. Então: $2 t \equiv 0\left(\bmod 2^{m}\right)$, $\operatorname{logo} t=0$ ou $t=2^{m-1}$. Conseqüentemente $A$ deve ser de um dos seguintes tipos:

$$
\begin{array}{r}
h^{2^{m}}=1, g^{2^{n}}=1, g h g^{-1}=h^{-1} \\
h^{2^{m}}=1, g^{2^{n}}=h^{2^{m-1}}, g h g^{-1}=h^{-1}
\end{array}
$$

No caso 3.3, o subgrupo cíclico $\langle h\rangle$ não é um subgrupo normal de $\mathcal{U}_{1}(\mathbb{Z} A)$, pelo lema 3.7. Contradição. Portanto, $A$ só pode ser um grupo do tipo 3.4.

O elemento

$$
\begin{aligned}
y= & 1+\left(1+g+\cdots+g^{2^{m_{1}}-1}\right) h(1-g) \\
& =1+\left(1+g+\cdots+g^{2^{n}-1}\right)\left(1+h^{2^{m}-1}\right)\left(h-h^{-1}\right)
\end{aligned}
$$

pertence a $\mathcal{U}_{1}(\mathbb{Z} A)$. Então $y^{-1} h y=h^{i}$ e $i \not \equiv 0(\bmod 2)$. Além disso, da igualdade $h y=y h^{i}$ e comparando os elementos da base que pertencem as classes laterais 
$g\langle h\rangle$ e $\langle h\rangle$ obtemos que:

$$
\begin{array}{r}
\left(1+h^{2^{m-1}}\right)\left(1-h^{-2}\right)=\left(1+h^{2^{m-1}}\right)\left(h^{i+1}-h^{i-1}\right) \\
\left(1+h^{2^{m-1}}\right)\left(h^{2}-1\right)+h=\left(1+h^{2^{m-1}}\right)\left(h^{i+1}-h^{i-1}\right)+h^{i}
\end{array}
$$

Como $A$ não é um grupo quatérnion, $h^{2^{m-1}-2} \neq 1$ e comparando os elementos da base na equação 3.14 nós concluímos que: $h^{i+1+2^{m-1}}=1$ ou $h^{i+1}=1$. Suponhamos que $h^{i+1}=1$. Nesse caso, $h^{2^{m-1}}=h^{2^{m-1}+1-i}$ e $h^{2^{m-1}-2}=h^{2^{m-1}+i-1}$. Portanto, $i \equiv-1\left(\bmod 2^{m}\right)$. Substituindo o valor de $i$ na equação 3.11 concluiríamos que $h^{2^{m-1}-2}=1$ o que é impossível. Além disso, se $h^{2^{m-1}+i+1}=1$ então concluiríamos da equação 3.14 que $h^{2^{m-1}}=h^{i+1}$, o que implicaria que $2^{m-1}-1 \equiv i\left(\bmod 2^{m}\right)$ e novamente substituindo o valor de $i$ na equação 3.11 , obteríamos que $h^{2^{m-1}-2}=h^{2^{m-1}+2}=1$, contradizendo a hipótese.

(ii) $r \equiv 1+2^{m-1}\left(\bmod 2^{m}\right)$ e $m>2$

Nesse caso $1=g^{2^{m}}=h^{t 2^{m-n}}$ e $t=2^{n} t_{1}$. Tomando $g_{1}=h^{j} g$, nós obtemos que:

$$
g_{1}^{2}=h^{j}\left(g h^{j} g^{-1}\right) g^{2}=h^{2 j+2^{m-1}} g^{2}
$$

a qual implica que:

$$
g_{1} 2^{2^{n}}=h^{2^{n} j\left(1+2^{m-2}\right)+2^{n} t_{1}}
$$

pois $g^{2}$ é um elemento central de $A$. Se $j$ for uma solução da congruência $j\left(1+2^{m-2}\right)+t_{1} \equiv 0\left(\bmod 2^{m-n}\right)$ então $\left\langle g_{1}\right\rangle \cap\langle h\rangle=1$ e os elementos $g$ e $h$ não comutam. Contradição com o lema 3.7.

(iii) $r \equiv-1+2^{m-1}\left(\bmod 2^{m}\right)$ e $m>2$

Então como no caso 1 (c)ii, tome $t=2^{n} t_{1}$ e $\left(-2+2^{m-1}\right) t \equiv 0\left(\bmod 2^{m}\right)$. Portanto, obteríamos que $t \equiv 0\left(\bmod 2^{m-1}\right)$ e $g^{2^{n}}=h^{2^{m-1}} t_{2}=1$ se 2 divide $t_{2}$, onde $t=t_{2} 2^{m-1}$, o que é impossível.

Por outro lado, se 2 não divide $t_{2}$, então:

$$
g^{2^{n}}=h^{2^{m-1}} \quad e \quad y=1+\left(1+g+g^{2}+\cdots+g^{2^{n}-1}\right)\left(1+h^{2^{m-1}}\right) h(1-g)
$$

é um elemento de $\mathcal{U}_{1}(\mathbb{Z} A)$. É claro que este elemento pode ser representado como:

$$
y=1+\left(1+g+g^{2}+\cdots+g^{2^{n}-1}\right)\left(1+h^{2^{m-1}}\right)\left(h-h^{-1}\right) .
$$

Portanto, $y h y^{-1}=h^{i}$ e repetindo o raciocínio do caso $1(\mathrm{c})$ i nós obtemos uma contradição. 
Teorema 3.10. (Bovdi,[15], teorema 7) Seja $H$ um subgrupo periódico normal abeliano de $\mathcal{U}_{1}(\mathbb{Z} G)$ de um grupo periódico $G$. Se $G$ não contém um subgrupo quatérnion $Q$ tal que $Q \cap H$ é um grupo cíclico de ordem 4 , então $H$ pertence ao centro de $G$.

Demonstração: É uma conseqüência direta do teorema 3.2 e do teorema 3.9.

(2) Suponha agora que $G$ possua um subgrupo quatérnion $Q$ tal que $Q \cap H$ seja um grupo cíclico de ordem 4.

Sem perda de generalidade vamos supor então que $H \triangleleft \mathcal{U}_{1}(\mathbb{Z} G)$ e que $G$ possua uma subgrupo quatérnion $Q$ tal que $Q \cap H=\langle a\rangle$, onde $Q$ é dado pelas seguintes relações: $Q=\left\{\langle a, b\rangle \mid a^{4}=1, b^{2}=a^{2}, b^{-1} a b=a^{-1}\right\}$.

Vamos estudar, a seguir, algumas propriedades sobre $\mathcal{N}_{G}(Q)$ o normalizador de $Q$ em $G$. Nos lemas que se seguem suponhamos que $g$ seja um elemento de ordem $2^{s}$ que pertence a $\mathcal{N}_{G}(Q) \backslash Q$.

Lema 3.11. (Bovdi,[15], lema 9) O elemento b pertence ao normalizador de qualquer subgrupo cíclico $\langle c\rangle$ tal que $\langle a\rangle \cap\langle c\rangle=\{1\}$.

Demonstração: Como $G$ é um grupo de torção, podemos supor que $c$ possua ordem $t$. A seguir suponha que $b c b^{-1} \notin\langle c\rangle$. Então $y=\widehat{c} b(1-c) \neq 0$. Como $a$ e $c$ são comutativos pelo lema 2, temos que:

$(1-n y) a(1+n y)=a+n(a y-y a)=a+n\left(a-a^{3}\right) y$ que é um elemento trivial e pertence a $\langle a\rangle$ para todo inteiro $n$, o qual implica que $\left(a-a^{3}\right) y=0$. Conseqüentemente:

$$
\left(1-a^{2}\right) \sum_{i=1}^{t-1} c^{i} b=\left(1-a^{2}\right) \sum_{i=1}^{t-1} c^{i} b c .
$$

Como por hipótese, $\langle a\rangle \cap\langle c\rangle=\{1\}$, concluímos que os todos os elementos nos lados esquerdo e direito da igualdade são distintos entre si dois a dois. Segue então que $c b=c^{i} b c$ para algum $i$ e $c^{-i+1}=b c b^{-1}$. Contradição.

Relembramos que os três lemas a seguir não se encontram nas hipóteses da seção:

Definição 3.12. Seja $G$ um grupo arbitrário. Um elemento $u \in \mathbb{Q} G$ é dito ser algébrico se ele for a raiz de algum polinômio $p(X)$ com coeficientes inteiros. 
Lema 3.13. (Higman) Sejam $G$ grupo abeliano finito de ordem $n, \mathbb{Q} G=I_{1} \oplus \cdots \oplus I_{k}$, a decomposição do anel de grupo $\mathbb{Q} G$ como soma direta de corpos ciclotômicos, $m_{i}$ o número de classes de resíduos módulo $n$ em $I_{i}$ relativamente primos com $n, m$ o mínimo múltiplo comum de $m_{1}, \cdots, m_{k}$. Então para qualquer inteiro algébrico $u \in \mathcal{U}(\mathbb{Q} G)$ temos que $u^{m} \in \mathbb{Z} G$.

Demonstração: Considere $A=\mathbb{Q} G$ e $I_{A}$ o anel dos inteiros algébricos em $A$. Então temos que $\mathbb{Z} G \subseteq A$. Sendo $G$ um grupo finito temos que $I_{A}$ é um $\mathbb{Z}$-módulo finitamente gerado, donde segue pela proposição 1.25 que $\left[\mathcal{U}\left(I_{A}\right), \mathcal{U}(\mathbb{Z} G)\right]=m$ é finito. Desse fato segue imediatamente a tese.

Lema 3.14. Sejam $G$ um grupo finito e $e=\alpha_{0} 1+\cdots+\alpha_{n} a_{n}$ um elemento idempotente não-nulo de $\mathbb{C} G$, I o ideal a esquerda gerado por e $\in \mathbb{C} G$, p o número de autovalores iguais a 1 da matriz correspondente ao idempotente e através da representação regular de $\mathbb{C} G$. Então a dimensão da álgebra I sobre $\mathbb{C}\left(\right.$ dim $\left._{\mathbb{C}} I\right)$ é igual a p e $\alpha_{0}=\frac{p}{n}$.

Demonstração: Considere a decomposição de $\mathbb{C} G=I \oplus I_{1}$ em ideais a esquerda. Onde $I$ e $I_{1}$ são gerados, respectivamente, pelos idempotentes correspondentes $e$ e $1-e$.

Vamos escolher uma base de $\mathbb{C} G$ formada pelos vetores $u_{1}, \cdots, u_{s}, v_{1}, \cdots, v_{r}$ de tal forma que os vetores $u_{1}, \cdots, u_{s}$ formem uma base de $I$ e os vetores $v_{1}, \cdots, v_{r}$ formem uma base de $I_{1}$.

Como $e_{1} e=0\left(e_{1}=1-e\right), e^{2}=e$ então temos que $u_{i} e=u_{i}(i=1, \cdots, s), v_{j} e=0(j=$ $1, \cdots, r)$. O que significa que ao elemento $e$ na base $u_{1}, \cdots, u_{s}, v_{1}, \cdots, v_{r}$ corresponde uma matriz

$$
\mathbf{E}^{\prime}=\left(\begin{array}{cccccc}
1 & & & & & \\
& \ddots & & & & \\
& & 1 & & & \\
& & & 0 & & \\
& & & & \ddots & \\
& & & & & 0
\end{array}\right)
$$

dos autovalores que são iguais a 1. Calculando o traço de $\alpha$ de duas maneiras obtemos por um lado que o traço de $\alpha$ é igual a $\alpha_{0} n$ e por outro lado, temos que traço de $\alpha(=$ traço de $E^{\prime}$ ) é igual a $p$. Desta forma $s=p$ e $\alpha_{0}=\frac{p}{n}$. Concluindo a demonstração do lema. 
Lema 3.15. Seja $G$ um grupo que pode ser representado como o produto direto dos grupos cíclico $T_{1}$ e $T_{2}$ de ordens, respectivamente, 4 e p, onde p é um inteiro primo maior que $3, T_{1}$ e $T_{2}$ gerados, respectivamente, pelos elementos c e a. Sejam $\xi$ uma raiz primitiva $4 p-e ́ s i m a$ da unidade, s o número de classes de resíduos módulo $4 p$ no corpo $\mathbb{Q}(\xi)$, relativamente primas com $4 p$. Então existe um inteiro $s$ tal que:

$$
\begin{aligned}
x & =1-\frac{1}{2 q}\left(1-c^{2}\right)[p-\widehat{a}]+\frac{1}{2 q}\left(1+a c+a^{2} c^{2}\right)^{s}\left(1-c^{2}\right)[p-\widehat{a}]= \\
& =\Lambda_{0}+\Lambda_{1} c+\Lambda_{2} c^{2}+\Lambda_{3} c^{3} \quad\left(\Lambda_{i} \in \mathbb{Q} T_{2} ; 0 \leq i \leq 3\right)
\end{aligned}
$$

é um elemento invertível no anel de grupo $\mathbb{Z} G$, além disso, valem as relações:

$$
\Lambda_{i} \neq 0(i=0,1,3), \Lambda_{3}=-\Lambda_{1} \quad \text { e } \quad \Lambda_{j} \neq \pm \Lambda_{0}(j=1,2,3) .
$$

Demonstração: Por hipótese temos que $G=\langle c\rangle \times\langle a\rangle$. Conseqüentemente temos pelo lema 1.28:

$$
\mathbb{Q} G=\mathbb{Q}(\langle c\rangle \times\langle a\rangle) \cong \mathbb{Q}(\langle c\rangle) \otimes_{\mathbb{Q}} \mathbb{Q}(\langle a\rangle)
$$

Pela proposição 1.26 temos que:

$$
\mathbb{Q}(\langle c\rangle) \cong \mathbb{Q} \oplus \mathbb{Q} \oplus \mathbb{Q}(i)
$$

e que

$$
\mathbb{Q}(\langle a\rangle) \cong \mathbb{Q} \oplus \mathbb{Q}\left(\zeta_{p}\right),
$$

onde $i$ representa a unidade imaginária e $\zeta_{p}$ uma raiz primitiva $p$-ésima da unidade.

Observe que de 3.9 e 3.10 em 3.8, obtemos que:

$\mathbb{Q} G \cong 2 \mathbb{Q} \oplus 2\left(\mathbb{Q} \otimes_{\mathbb{Q}} \mathbb{Q}\left(\zeta_{p}\right)\right) \oplus \mathbb{Q}(i) \otimes_{\mathbb{Q}} \mathbb{Q} \oplus \mathbb{Q}(i) \otimes_{\mathbb{Q}} \mathbb{Q}\left(\zeta_{p}\right) \cong 2 \mathbb{Q} \oplus 2 \mathbb{Q}\left(\zeta_{p}\right) \oplus \mathbb{Q}(i) \oplus \mathbb{Q}(i) \otimes_{\mathbb{Q}} \mathbb{Q}\left(\zeta_{p}\right)$

Considere a seguir a álgebra de grupo $\mathbb{Q}(\langle a\rangle)$. Logo utilizando a proposição 1.11 e o lema 1.10 temos que:

$$
\mathbb{Q}(\langle a\rangle) \cong \mathbb{Q}(\langle a\rangle)\left(\frac{\widehat{a}}{p}\right) \oplus \mathbb{Q}(\langle a\rangle)\left(1-\frac{\widehat{a}}{p}\right),
$$

onde $g_{1}=\frac{\widehat{a}}{p}$ e $g_{2}=1-\frac{\widehat{a}}{p}$ são os idempotentes centrais primitivos da álgebra de grupo $\mathbb{Q}(\langle a\rangle)$.

Considere a seguir a álgebra de grupo $\mathbb{Q}(\langle c\rangle)$. Temos pelo teorema do isomorfismo para anéis e pela proposição 1.26 que:

$$
\frac{Q(\langle c\rangle)}{\triangle\left(\langle c\rangle,\left\langle c^{2}\right\rangle\right)} \cong \mathbb{Q}\left(\frac{\langle c\rangle}{\left\langle c^{2}\right\rangle}\right) \cong \mathbb{Q} \oplus \mathbb{Q} .
$$


Pela proposição 1.11 e pelo lema 1.10 temos que

$$
\triangle\left(\langle c\rangle,\left\langle c^{2}\right\rangle\right)=\mathbb{Q}(\langle c\rangle)\left(\frac{1-c^{2}}{2}\right) .
$$

Além disso, obtemos que:

$$
\mathbb{Q}(\langle c\rangle)\left(\frac{1+c^{2}}{2}\right) \cong \mathbb{Q} \oplus \mathbb{Q} .
$$

Donde obtemos os idempotentes

$$
f_{1}=\frac{\widehat{c}}{4} \quad \text { e } \quad f_{2}=\left(\frac{1+c^{2}}{2}-f_{1}\right)=\frac{1-c+c^{2}-c^{3}}{4} .
$$

Podemos concluir daí que $f_{1}, f_{2}$ e $f_{3}=\frac{1-c^{2}}{2}$ são os idempotentes centrais primitivos da álgebra de grupo $\mathbb{Q}(\langle c\rangle)$.

De 3.11 e utilizando os idempotentes das álgebras de grupo $\mathbb{Q}(\langle c\rangle)$ e $\mathbb{Q}(\langle a\rangle)$ obtidos em 3.12 e 3.14 podemos obter os idempotentes da álgebra de grupo $\mathbb{Q} G$, os quais são dados por:

$$
\begin{array}{r}
e_{1}=\frac{1}{4 p} \widehat{c} \widehat{a} ; \\
e_{2}=\frac{1}{4 p}\left(1-c+c^{2}-c^{3}\right) \widehat{a} ; \\
e_{3}=\frac{1}{2 p}\left(1-c^{2}\right) \widehat{a} ; \\
e_{4}=\frac{1}{4 p} \widehat{c}(p-\widehat{a}) ; \\
e_{5}=\frac{1}{4 p}\left(1-c+c^{2}-c^{3}\right)(p-\widehat{a}) ; \\
e_{6}=\frac{1}{2 p}\left(1-c^{2}\right)(p-\widehat{a}) .
\end{array}
$$

O que implica que $\mathbb{Q} G$ possui uma decomposição em soma direta de corpos ciclotômicos da forma:

$$
\mathbb{Q} G=I_{1} \oplus \cdots \oplus I_{6} .
$$

O elemento ace $_{6}$ em $I_{6}$ é uma raiz primitiva $4 p$-ésima da unidade. Na verdade, é claro que $\left(a c e_{6}\right)^{m}=a^{m} c^{m} e_{6}=e_{6}$ se e somente se $m \equiv 0(\bmod 4 p)$.

Pelo lema 3.14 temos que a dimensão de $I_{6}$ sobre $\mathbb{Q}$ ( observe que $\left\{\alpha e_{6} \mid \alpha \in \mathbb{Q}\right\}=\mathbb{Q} e_{6} \cong \mathbb{Q}$ ) é igual a $2(p-1)=\varphi(4 p)$, onde $\varphi$ denota a função de Euler. Isso implica que $I_{6}$ é isomorfo a $\mathbb{Q}(\xi)$. É claro que $\frac{1-\xi^{3}}{1-\xi}=1+\xi+\xi^{2}$ é uma unidade em $\mathbb{Z}(\xi)$ pois a equação $3 x \equiv 1$ $(\bmod 4 p)$ admite solução (visto que $p>3)$. 


\section{Caracterização dos subgrupos normais de torção de $\mathcal{U}_{1}(\mathbb{Z} G)$ de um grupo periódico}

Conseqüentemente temos que $\left(1+a c+a^{2} c^{2}\right) e_{6}$ é uma unidade inteira em $I_{6}$. O que por sua vez implica que $y=e_{1}+\cdots+e_{5}+\left(1+a c+a^{2} c^{2}\right) e_{6}$ é uma unidade inteira de $\mathbb{Q} G$. Segue pelo lema 3.13 que existe um inteiro $s$ tal que:

$$
\begin{gathered}
u=y^{s}=e_{1}+\cdots+e_{5}+\left(1+a c+a^{2} c^{2}\right)^{s} e_{6}= \\
=1-\frac{1}{2 p}\left(1-c^{2}\right)((p-\widehat{a})+ \\
+\frac{1}{2 p}\left(1+a c+a^{2} c^{2}\right)^{s}\left(1-c^{2}\right)(p-\widehat{a})
\end{gathered}
$$

é uma unidade em $\mathbb{Z} G$.

Consideremos

$$
\begin{gathered}
\frac{1}{2 p}\left((p-1)-a-\cdots-a^{p-1}\right)=A ;\left(1+a c+a^{2} c^{2}\right)^{s}=B_{0}+B_{1} c+B_{2} c^{2}+B_{3} c^{3} \\
\left(B_{i} \in \mathbb{C} T_{2}, i=0, \cdots, 3\right) .
\end{gathered}
$$

Então:

$$
u=\Lambda_{0}+\Lambda_{1} c+\Lambda_{2} c^{2}+\Lambda_{3} c^{3}
$$

onde,

$$
\begin{array}{r}
\Lambda_{0}=\left(B_{0}-B_{2}+1\right) A+1 ; \Lambda_{1}=\left(B_{1}+B_{3}\right) A ; \\
\Lambda_{2}=\left(B_{2}-B_{0}+1\right) A ; \Lambda_{3}=\left(B_{3}-B_{1}\right) A .
\end{array}
$$

De 3.15 segue imediatamente que $\Lambda_{0} \neq 0 ; \Lambda_{i} \neq \pm \Lambda_{0}(i=1,2,3)$, caso contrário a unidade do anel de grupo $\mathbb{Q} T_{2}$ pertenceria ao ideal gerado pelo idempotente $\frac{1}{p}(p-\widehat{a})$, o que é uma contradição. O fato que $\Lambda_{3}=-\Lambda_{1}$ é trivial.

Suponhamos agora que $\Lambda_{1}=0$ então teríamos que $\Lambda_{3}=0$ e $u=\Lambda_{0}+\Lambda_{2} c^{2}$. Elevando o elemento $1+\xi+\xi^{2}$ a potência $s$ (e tomando os graus módulo $4 p$ ) obtemos que:

$$
\left(1+\xi+\xi^{2}\right)^{s}=\xi^{2 s}+\cdots
$$

Ou seja, concluímos que não existem termos $\xi$ com grau ímpar no lado direito da igualdade acima, o que implica que

$$
\left(1+\xi+\xi^{2}\right)^{s}=\left(1-\xi+\xi^{2}\right)^{s}
$$

Segue de 3.18 que:

$$
\left|1+\xi+\xi^{2}\right|=\left|1-\xi+\xi^{2}\right|
$$

No entanto, isso não pode ocorrer, visto que os vetores $\xi$ e $1+\xi^{2}$ são linearmente dependentes sobre $\mathbb{C}$. Obtemos uma contradição, concluindo a demonstração do lema. 
Lema 3.16. (Bovdi,[15], lema 10) O normalizador $\mathcal{N}_{G}(Q)$ do subgrupo dos quatérnions de ordem 8 não possui elementos de ordem ímpar.

Demonstração: Suponha que $g \in \mathcal{N}_{G}(Q)$ e $g$ seja um elemento de ordem prima $q$.

Os únicos casos possíveis são:

(a) $g b=b g$ e $q=3$.

Nesse caso seja $L=\langle g\rangle \times\langle b\rangle$. Então $x=1+\left[225\left(2-g-g^{2}\right)+390\left(b g^{2}-b g\right)\right]\left(1-b^{2}\right)$ é um elemento invertível do anel de grupo $\mathbb{Z} L$, sendo seu inverso dado por: $x^{-1}=$ $1+\left[225\left(2-g-g^{2}\right)-390\left(b g^{2}-b g\right)\right]\left(1-b^{2}\right)$. Pelo teorema $3.5, x^{-1} a x \in\langle a\rangle$, no entanto, verificando diretamente concluímos que $a x \neq x a$ e $a x \neq x a^{3}$. Contradição.

(b) $g b=b g$ e $q>3$. Sejam $\xi$ uma raiz primitiva $4 q$-ésima da unidade e $s$ o número de classes de restos módulo $4 q$ no corpo $\mathbb{Q}(\xi)$ as quais são relativamente primas com $4 q$. Então, pelo lema 3.15 temos que existe um inteiro $s$ tal que:

$$
x=1-\frac{1}{2 q}\left(1-b^{2}\right)[q-\widehat{g}]+\frac{1}{2 q}\left(1+g b+g^{2} b^{2}\right)^{s}\left(1-b^{2}\right)[q-\widehat{g}]
$$

é um elemento invertível no anel de grupo $\mathbb{Z} L$.

Podemos escrever $x$ na forma:

$$
x=\Lambda_{0}+\Lambda_{1} b+\Lambda_{2} b^{2}+\Lambda_{3} b^{3},
$$

onde os $\Lambda_{i}$ são elementos do anel de grupo inteiro do grupo cíclico $\langle g\rangle$, satisfazendo as relações: $\Lambda_{i} \neq 0(i=0,1,3), \Lambda_{3}=-\Lambda_{1}$ e $\Lambda_{j} \neq \pm \Lambda_{0}(j=1,2,3)$.

Pelo teorema 3.5 temos que $a x=x a$ ou $x a=a^{3} x$. Se $a x=x a$ então $\Lambda_{1}=\Lambda_{3}$, e se $x a=a^{3} x$ então obtemos que $\Lambda_{0}=\Lambda_{2}$, as quais contradizem a relação anterior.

(c) $g b \neq b g$.

Pelo lema 3.11, temos que $g b=b g^{j}(j>0)$ e como $b^{2}=a^{2}$, temos que:

$$
g=b^{-2} g b^{2}=g^{j^{2}} .
$$

Portanto, $j^{2} \equiv 1(\bmod q)$ donde $j \equiv q-1(\bmod q)$ ou $j \equiv 1(\bmod q)$.

Se $j \equiv 1(\bmod q)$ então concluiríamos que $g$ e $b$ comutam. Contradição. Suponhamos agora que $j \equiv q-1(\bmod q)$. Nesse caso, obteríamos que:

$$
g b=b g^{q-1} .
$$

Por outro lado, temos que $g \in \mathcal{N}_{G}(Q)$, o qual implica que $g b=b a^{i} g$ e comparando com 3.20, obtemos que $b g^{-1}=b a^{i} g$, donde $g^{2}=a^{-i}$. O que é impossível. 
Lema 3.17. (Bovdi,[15], lema 12) Os elementos de ordem impar em $G$ formam um subgrupo normal abeliano $L, b^{-1} g b=g^{-1}$, para qualquer $g \in L$; e em particular, todo subgrupo de $L$ é normal em $G$.

Demonstração: Mostraremos inicialmente que todo subgrupo cíclico $\langle c\rangle$ de ordem ímpar $k$ de $G$ é normal em $G$.

Suponhamos que $c^{\prime}=d^{-1} c d \notin\langle c\rangle$, para algum $c^{\prime} \in G$ e que $c$ possua ordem $p_{1}^{\alpha_{1}} p_{2}^{\alpha_{2}} \cdots p_{s}^{\alpha_{s}}$, onde $p_{i}, 1 \leq i \leq s$, primos distintos. Pelo lema 3.7, os elementos $a$ e $c$ comutam, e pelo lema 3.11, $b \in \mathcal{N}_{G}(\langle c\rangle)$. Podemos representar $c$ na forma $c=c_{1} c_{2} \cdots c_{s}$, onde cada $c_{i}$ possui ordem $p_{i}^{\alpha_{i}}$. Pelo lema $3.11, b \in \mathcal{N}_{G}\left(\left\langle c_{i}\right\rangle\right)$ e da equação $b c_{i} b^{-1}=c_{i}^{e}($ observe que $b \widehat{c}=\widehat{c} b)$ segue que $e^{2} \equiv 1\left(\bmod p_{i}^{\alpha_{i}}\right)$ e pelo lema 3.16 obtemos que $e=-1$. Logo $b^{-1} c b=c^{-1}$.

Aplicando o teorema 3.5 ao subgrupo cíclico $\langle a\rangle$, temos as seguintes possibilidades:

(a) $d^{-1} a d=a$

Nesse caso, $y=b \widehat{c} d(1-c)$ é um elemento nilpotente não-nulo, $y^{2}=0$ (pois $b \widehat{c}=\widehat{c} b$ ). Além disso, $(1+n y) a(1+n y)=a+\left(a^{3}-a\right) y n$ pertence a $\langle a\rangle$ para qualquer inteiro $n$, o que é possível se $\left(a^{3}-a\right) y=0$. Segue daí que: $a^{2} \widehat{c}\left(1-c^{\prime}\right)=\widehat{c}\left(1-c^{\prime}\right)$ e comparando os dois lados da igualdade concluímos que $a^{2}=c^{i}$ para algum $i$. Contradição.

(b) $d^{-1} a d=a^{3}$

Nesse caso, considerando $y=\widehat{c} d(1-c)$ e repetindo o raciocínio anterior, obtemos uma contradição.

Teorema 3.18. (Bovdi,[15], teorema 10) Suponha que o subgrupo abeliano periódico $H$ de $G$ é um subgrupo normal de $\mathcal{U}_{1}(\mathbb{Z} G)$, e $G$ contém um subgrupo quatérnion $Q$ tal que $Q \cap H=\langle a\rangle$ seja um grupo cíclico de ordem 4. Então:

(a) O centralizador $C_{S_{2}}(\langle a\rangle)$ do subgrupo normal cíclico $\langle a\rangle$ num 2-subgrupo de Sylow $S_{2} \subseteq G$, o qual contém o subgrupo dos quatérnions $Q$, é um subgrupo normal abeliano de indice 2, e para qualquer $x \in S_{2}, x \notin C_{S_{2}}(\langle a\rangle), x^{2}=a^{2}$ e $x^{-1} y x=y^{-1},(y \in$ $\left.C_{S_{2}}(\langle a\rangle)\right)$.

(b) Os elementos de ordem impar em $G$ formam um subgrupo abeliano normal $L$, todo subgrupo de $L$ é um subgrupo normal de $G$, e $x^{-1} y x=y^{-1}(y \in L)$ para qualquer 2-elemento $x$ que não pertença a $C_{S_{2}}(\langle a\rangle)$.

(c) O subgrupo normal $H$ coincide com o grupo cíclico $\langle a\rangle$ de ordem 4 ou pode ser representado como o produto direto de $\langle a\rangle$ e um grupo com expoente dois. 
Demonstração: Seja $S_{2}$ um 2-subgrupo de Sylow de $G$ que contém um subgrupo quatérnion $Q$ tal que o grupo $Q \cap H$ seja cíclico de ordem 4. Então tomando $N=C_{S_{2}}(\langle a\rangle)$, temos que $N$ é um subgrupo normal de $S_{2}$ de índice 2 pelo teorema 3.5 , visto que $b \notin N$.

Afirmamos inicialmente que se $h$ é um elemento de ordem $2^{s}$ em $S_{2}$ então $h^{2}=a^{2}$.

Pelo lema 3.7, temos que $\langle a\rangle \cap\langle h\rangle$ é um grupo cíclico de ordem 2. Se $s>2$ então considere $A=\langle a, h\rangle$. Como o elemento $h^{2}$ é central em $A$, temos que o subgrupo $B=\langle a\rangle\left\langle h^{2}\right\rangle$ é um subgrupo normal abeliano de $\mathcal{U}_{1}(\mathbb{Z} A)$. Nesse caso podemos decompor $B$ como $B=$ $\left\langle h^{2}\right\rangle \times\left\langle h^{\prime}\right\rangle$, onde $\left\langle h^{\prime}\right\rangle$ é cíclico de ordem 2. Pelo teorema 3.5 concluímos que $\left\langle h^{\prime}\right\rangle$ é normal em $\mathcal{U}_{1}(\mathbb{Z} A)$. Contradição, pois os elementos $a^{\prime}$ e $h$ não comutam. Portanto, concluímos que $s=2$. E se $g \in N$ e $h \in S_{2} \backslash N$ temos que $(h g)^{2}=a^{2}$ ou seja, $h g h^{-1}=g^{-1}$.

Afirmamos a seguir que o grupo $N$ é abeliano.

Sejam $c$ e $d$ elementos de $N$. Da relação que vimos anteriormente temos que:

$$
c d=b^{-1}(c d)^{-1} b=b^{-1} d^{-1} c^{-1} b=(d b)^{-1} c^{-1} d^{-1}(d b)=d c .
$$

Seja

$$
H=W_{2} \times W_{p_{1}} \times \cdots \times W_{p_{t}},
$$

a decomposição do grupo $H$ no produto direto dos seus $p$-subgrupos de Sylow. $W_{p i}$ é um subgrupo normal de $\mathcal{U}_{1}(\mathbb{Z} G)$ e o centralizador $W_{p}(p \neq 2)$ contém todos os elementos de ordem finita em $G$ conforme a demonstração do teorema 3.9. Contradição com o lema 3.17 , donde concluímos que $H=W_{2}$.

Consideremos agora $A=\langle b, h\rangle, h \in H, b^{-1} h b=h^{-1}$. Segue pelo teorema 2 que o subgrupo $\langle h\rangle$ é normal em $\mathcal{U}_{1}(\mathbb{Z} A)$, o que é possível, somente se $h^{2}=1$ ou $h^{2}=a^{2}$ conforme a demonstração do teorema 3.9 .

\subsubsection{Estrutura dos grupos periódicos $G$ tais que $\mathcal{U}_{1}(\mathbb{Z} G)$ contém um subgrupo normal periódico abeliano não-central}

Definição 3.19. Diremos que $G$ é um $Q^{*}$-grupo se $G$ possuir um subgrupo normal abeliano $L$ de índice 2 com um elemento a de ordem 4 tal que para todo $g \notin L, g^{2}=\boldsymbol{a}^{2}$ e $g^{-1} h g=h^{-1}$, para todo $h$ em $L$.

Teorema 3.20. (Bovdi, [15], teorema 11) Seja $G$ um grupo periódico. $\mathcal{U}_{1}(\mathbb{Z} G)$ contém um subgrupo periódico normal abeliano não central se e somente se $G$ for um $Q^{*}$-grupo.

Demonstração: Suponha que $\mathcal{U}_{1}(\mathbb{Z} G)$ contém um subgrupo periódico normal abeliano não central. Então o resultado é uma conseqüência dos teoremas 3.2, 3.10 e 3.18. 


\section{Caracterização dos subgrupos normais de torção de $\mathcal{U}_{1}(\mathbb{Z} G)$ de um grupo periódico}

46

Reciprocamente, mostraremos que $\langle a\rangle \triangleleft \mathcal{U}$.

Qualquer que seja $x \in \mathcal{U}(\mathbb{Z} G), x$ pode ser escrito como $x=x_{1}+x_{2} b$, onde $x_{1}$ e $x_{2} \in \mathbb{Z} L, b \notin L$.

Utilizando as propriedades de involução, temos que:

$a x x^{*}=a\left(x_{1}+x_{2} b\right)\left(x_{1}+x_{2} b\right)^{*}=a\left(x_{1}+x_{2} b\right)\left(x_{1}^{*}+b^{*} x_{2}^{*}\right)=a\left(x_{1}+x_{2} b\right)\left(x_{1}^{*}+b^{-1} x_{2}^{*}\right)=$ $a\left(x_{1}+x_{2} b\right)\left(x_{1}^{*}+x_{2} b^{-1}\right)=a\left(x_{1} x_{1}^{*}+x_{1} x_{2} b^{-1}+x_{2} b x_{1}^{*}+x_{2} b x_{2} b^{-1}\right)=a\left(x_{1} x_{1}^{*}+x_{1} x_{2} b^{3}+b b^{-1} x_{2} b x_{1}^{*}+\right.$ $\left.x_{2} b x_{2} b^{-1}\right)=a\left(x_{1} x_{1}^{*}+x_{1} x_{2} b^{3}+b x_{2}^{*} x_{1}^{*}+x_{2} x_{2}^{*}\right)=a\left(x_{1} x_{1}^{*}+x_{1} x_{2} b^{3}+b\left(x_{1} x_{2}\right)^{*}+x_{2} x_{2}^{*}\right)=a\left(x_{1} x_{1}^{*}+\right.$ $\left.x_{1} x_{2} b^{3}+x_{1} x_{2} b+x_{2} x_{2}^{*}\right)=a\left(x_{1} x_{1}^{*}+x_{2} x_{2}^{*}+x_{1} x_{2}\left(1+a^{2}\right) b\right)=x x^{*} a$, pois $b x b^{-1}=x^{*}$, para todo $x \in \mathbb{Z} L$.

Ou seja, mostramos que: $a x x^{*}=x x^{*} a$.

Utilizando a relação acima temos:

$$
\left(x^{-1} a x\right)\left(x^{-1} a x\right)^{*}=x^{-1} a x x^{*} a^{*}\left(x^{-1}\right)^{*}=x^{-1} a x x^{*} a^{3}\left(x^{*}\right)^{-1}=x^{-1} x x^{*} \underbrace{a a^{3}}_{=1}\left(x^{*}\right)^{-1}=1 .
$$

Pelo lema 1.17, aplicada a igualdade acima concluímos que

$$
x^{-1} a x=g \in G .
$$

Afirmamos que $g \in L$.

Suponhamos, por absurdo, que $g \notin L$.Como $L$ é um subgrupo de $G$ de índice $2, g$ pode expresso na forma $g=h b$, para algum $h \in L$.

E da igualdade $a\left(x_{1}+x_{2} b\right) \underbrace{=}_{5.12}\left(x_{1}+x_{2} b\right) g=x_{1} g+x_{2} b g=x_{1} h b+x_{2} h^{-1} a^{2}$.

Nós obtemos então:

$$
\begin{aligned}
& a\left(x_{1}+x_{2} b\right)=x_{1} h b+x_{2} h^{-1} a^{2} \\
& x_{1}+x_{2} b=a^{-1} x_{1} h b+a^{-1} x_{2} h^{-1} a^{2}
\end{aligned}
$$

Donde concluímos que: $x_{1}=a^{-1} x_{2} h^{-1} a^{2}$.

Aplicando o homomorfismo de aumento $\varepsilon$ a unidade $x$, nós obtemos:

$$
1=\varepsilon(x)=\varepsilon\left(a^{-1} x_{2} h^{-1} a^{2}\right)+\varepsilon\left(x_{2} b\right)=\varepsilon\left(a^{-1}\right) \varepsilon\left(x_{2}\right) \varepsilon\left(h^{-1}\right) \varepsilon\left(a^{2}\right)+\varepsilon\left(x_{2}\right) \varepsilon(b)=2 \varepsilon\left(x_{2}\right)
$$

O que é absurdo pois $\varepsilon\left(x_{2}\right) \in \mathbb{Z}$.

Portanto, $g \in L$ e $g^{2}=a^{2}$.

É fácil de observar que o subgrupo cíclico $\langle a\rangle$ é normal em $G$.

Seja $\psi: \mathbb{Z} G \longmapsto \mathbb{Z}(G /\langle a\rangle)$, epimorfismo canônico. Obtemos então que $\psi(g)=\psi\left(x^{-1} a x\right)=\overline{1}$. Logo $g \in \operatorname{Ker} \psi \cap G=\langle a\rangle$. Assim temos que $g \in\left\{a, a^{-1}\right\}$, pois $a$ é um elemento de ordem 4 . Portanto, podemos concluir daí que $x^{-1} a x=a$ ou $x^{-1} a x=a^{3}$ e a tese segue. 


\subsection{Um teorema de Bovdi}

Sejam $G$ um grupo periódico e $\mathcal{U}$ o respectivo grupo de unidades de $\mathbb{Z} G$. Nessa seção utilizaremos os resultados obtidos nas seções anteriores com o objetivo de obtermos uma caracterização dos grupos periódicos $G$ em função do subgrupo dos elementos de torção do hipercentro de $\mathcal{U}_{1}(\mathbb{Z} G)$.

Seja $G$ um grupo periódico e seja

$$
(1)=Z_{0}(\mathcal{U}) \leq Z_{1}(\mathcal{U}) \leq \cdots \leq Z_{n}(\mathcal{U}) \leq \cdots
$$

a série central superior do grupo de unidades $\mathcal{U}$.

Denotaremos por $\widetilde{Z}(\mathcal{U})$ o hipercentro de $\mathcal{U}$. Relembramos também que $\widetilde{Z}(\mathcal{U})$ é um subgrupo normal de $\mathcal{U}$, além disso, se $T=T(\widetilde{Z}(\mathcal{U}))$ denotar o conjunto de todas as unidades de torção em $\widetilde{Z}(\mathcal{U})$ com aumento 1 , então $T$ é um subgrupo normal periódico de $\mathcal{U}$.

Passamos então a enunciar o resultado principal desse capítulo.

Teorema 3.21. ([Bovdi][14] e [15]) Seja G um grupo periódico. Então ocorre exatamente um dos seguintes casos:

(1) G é um 2-grupo Hamiltoniano e $T=G$;

(2) $T=Z_{1}(G)$;

(3) $G$ possui um subgrupo normal $H$ de indice 2 contendo um elemento a de ordem 4 tal que para cada $g \in G \backslash H, g^{2}=a^{2}$ e ghg $g^{-1}=h^{-1}$ para todo $h \in H$, e $T=\langle a\rangle \oplus E=$ $Z_{2}(\mathcal{U}) \cap Z_{2}(G)$ onde $E$ é um 2-grupo abeliano elementar.

Observação 3.22. Ressaltamos que se $G$ é um $Q^{*}$-grupo então $Z_{1}(G)=\left\{y \in G \mid y^{2}=1\right\}$ e o expoente de $T$ é 4 , onde $T$ denota o subgrupo dos elementos de torção do hipercentro de $\mathcal{U}$.

Observação 3.23. Utilizando a proposição 1.30, concluímos que no caso (1) do Teorema 3.21, $G=Q \times E$, onde $Q$ é o grupo dos quatérnions de ordem 8 e E é um 2-grupo abeliano elementar. Além disso, o lema 1.19 nos diz que $\mathcal{U}= \pm G$.

Vamos agora provar o teorema 3.21.

Demonstração: Já vimos que $T$ é um subgrupo normal periódico de $\mathcal{U}$. Pelo teorema 3.2 concluímos que todo subgrupo de $T$ é normal em $G$, em particular, o próprio $T$. 
Caracterização dos subgrupos normais de torção de $\mathcal{U}_{1}(\mathbb{Z} G)$ de um grupo periódico 48

Se $T$ não for abeliano e sendo $G$ um grupo periódico, segue do teorema 3.8 que $G$ deve ser um 2-grupo Hamiltoniano.

Suponhamos, agora, que $T$ seja abeliano. Nesse caso, podem ocorrer dois fatos:

- $T \leq Z_{1}(G)$, e nesse caso a condição (2) do teorema 3.21 é satisfeita, ou

- Pelo teorema 3.20, $T$ deve ser um grupo do tipo mostrado em (3) no enunciado do teorema 3.21 , e neste caso $\langle a\rangle \triangleleft \mathcal{U}$, ( conforme a recíproca na demonstração do teorema 3.20), e portanto, $a \in Z_{2}(U)$. De fato, considere a projeção natural $\varphi: \mathcal{U} \longmapsto \mathcal{U} / \mathcal{Z}(\mathcal{U}):=\overline{\mathcal{U}}$, onde $\varphi(a)=\bar{a}$. Como $\langle a\rangle \triangleleft \mathcal{U}$, então $\langle\bar{a}\rangle \triangleleft \overline{\mathcal{U}}$ e como $a^{2} \in \mathcal{Z}(\mathcal{U})$ segue que $o(\bar{a})=2$ logo, $\bar{a} \in \mathcal{Z}(\overline{\mathcal{U}})$. Portanto, $\langle a\rangle \subseteq \varphi^{-1}(\langle\bar{a}\rangle) \subseteq \varphi^{-1}(\mathcal{Z}(\overline{\mathcal{U}}))=: Z_{2}(\mathcal{U})$. Onde $\varphi^{-1}(\mathcal{Z}(\overline{\mathcal{U}}))$ indica a imagem inversa de $\mathcal{Z}(\overline{\mathcal{U}})$ em $\mathcal{U}$. Logo $a \in Z_{2}(\mathcal{U})$.

A estrutura de $T$ segue do teorema 3.18(c) e é claro que $T \leq Z_{2}(G)$. Como todos os elementos de $G$ possuindo ordem 2 são centrais em $\mathcal{U}$, concluímos que $T \leq Z_{2}(\mathcal{U})$ e portanto, $T=Z_{2}(\mathcal{U}) \cap Z_{2}(G)$. 


\section{Capítulo 4}

\section{A altura central de $\mathcal{U}_{1}(\mathbb{Z} G)$ de um grupo finito}

Ressaltamos que neste capítulo, salvo menção em contrário, o grupo $G$ será sempre um grupo finito.

Neste capítulo passamos a estudar a série central superior de $\mathcal{U}=\mathcal{U}_{1}(\mathbb{Z} G)$, de um grupo finito $G$, com o objetivo de obtermos mais informações sobre a estrutura de $\mathcal{U}_{1}(\mathbb{Z} G)$.

Já vimos no capítulo 2 que o teorema de Gruenberg desempenha um papel fundamental em nosso estudo pois nos garantirá que a altura central de $\mathcal{U}$ é finita. Esse resultado é crucial e justifica o estudo do hipercentro de $\mathcal{U}$.

No entanto, ainda surgem algumas questões que desejamos discutir neste capítulo. A altura central de $\mathcal{U}$ sendo finita, dependeria do grupo $G$ em questão? Seria possível, caracterizarmos os grupos $G$ em função da altura central de $\mathcal{U}$ ?

Tendo em mente essas questões, nossos objetivos principais nesse capítulo serão: Mostrar que a altura central de $\mathcal{U}$ é finita; obter uma caracterização para o hipercentro de $\mathcal{U}$ em função do subgrupo de torção do hipercentro de $\mathcal{U}$; caracterizarmos os grupos $G$ em função da altura central de $\mathcal{U}$; além de procurarmos mostrar a relação entre a Conjectura do Normalizador e as unidades hipercentrais.

\subsection{A altura central de $\mathcal{U}_{1}(\mathbb{Z} G)$ é finita}

Demonstraremos aqui o resultado do Capítulo 2 quando enunciamos o teorema de Gruenberg. Veremos então como o teorema de Gruenberg e o teorema de Borel-Chandra nos garantirão que a série central superior de $\mathcal{U}_{1}(\mathbb{Z} G)$ estaciona. 
Antes de mais nada introduzimos algumas definições:

Definição 4.1. Sejam $R$ um domínio de integridade noetheriano com corpo de quocientes $K$.

Para qualquer $K$-espaço $V$ de dimensão finita, um $R$-lattice em $V$ é um $R$-módulo $M$ finitamente gerado tal que $K M=V$, onde

$$
K M=\left\{\sum \alpha_{i} m_{i}(\text { somas finitas }) \mid \alpha_{i} \in K, m_{i} \in M\right\} .
$$

Definição 4.2. Sejam $R$ um domínio de integridade noetheriano com corpo de quocientes $K$, A uma $K$-álgebra de dimensão finita.

Uma $R$-ordem numa $K$-álgebra $A$ é um subanel $\Lambda$ de $A$, possuindo o mesmo elemento unidade de $A$, tal que $\Lambda$ é um $R$-lattice em $A$.

Vamos supor, até o momento, o teorema conhecido de Borel-Chandra e apresentaremos uma idéia não rigorosa da demonstração desse teorema no apêndice da dissertação.

Teorema 4.3. (Borel-Chandra) Sejam A um $\mathbb{Q}$-álgebra de dimensão finita e $O$ uma $\mathbb{Z}$-ordem em A. Então o grupo de unidades de $O$ é finitamente gerado.

Teorema 4.4. Seja $G$ um grupo finito então $\mathcal{U}_{1}(\mathbb{Z} G)$ pode ser considerado como grupo linear finitamente gerado sobre $\mathbb{Z}$.

Demonstração: Mostraremos inicialmente que, via representação regular, $\mathcal{U}_{1}(\mathbb{Z} G)$ é um grupo linear sobre $\mathbb{Z}$.

Seja $T: \mathcal{U}_{1}(\mathbb{Z} G) \longmapsto M_{n}(\mathbb{Z})$ definida por $T(\alpha)=T_{\alpha}$, onde $T_{\alpha}: \mathbb{Z} G \longmapsto \mathbb{Z} G$ é definida por $T_{\alpha}(x)=\alpha x$, a representação regular de $\mathcal{U}_{1}(\mathbb{Z} G)$ e $n$ é a ordem do grupo $G$. Se $\alpha=$ $\sum_{g \in G} \alpha_{g} g$ então observamos que $T_{\alpha}(x)=\left(\sum_{g \in G} \alpha_{g} g\right)(x)=\sum_{g \in G} \alpha_{g}(g x)=\sum_{g \in G} \alpha_{g} T_{g}(x)$, ou seja observamos que a representação regular de $\mathcal{U}_{1}(\mathbb{Z} G)$ é totalmente caracterizada pela representação regular do grupo $G$.

Enumerando os elementos de $G$ de alguma maneira: $\left\{1, g_{1}, \cdots, g_{n}\right\}$ é fácil de observarmos que $T_{g}\left(g_{i}\right) \neq g_{i}$ para todo $g \neq 1$, o que nos mostra que a matriz de $T$ é uma matriz de permutação com traço zero. No caso em que $g=1$, obtemos que a matriz de $T$ é a matriz identidade de ordem $|G|$.

Não é difícil de observar que a representação regular é um monomorfismo. Segue desse fato e dos fatos mencionados anteriormente que podemos considerar $\mathcal{U}_{1}(\mathbb{Z} G)$ como grupo linear sobre $\mathbb{Z}$. Observe que neste caso, $\mathcal{U}_{1}(\mathbb{Z} G) \cong T\left(\mathcal{U}_{1}(\mathbb{Z} G)\right)$.

Sendo $G$ um grupo finito, temos que $\mathbb{Z} G$ é $\mathbb{Z}$-uma ordem em $\mathbb{Q} G$. Conseqüentemente, pelo teorema 4.3 temos que o grupo de unidades $\mathcal{U}_{1}(\mathbb{Z} G)$ é finitamente gerado. 
Dos fatos acima, segue imediatamente a tese.

Provaremos agora o resultado principal dessa seção.

Teorema 4.5. Seja $G$ um grupo finito então $\mathcal{U}_{1}(\mathbb{Z} G)$ possui altura central finita.

Demonstração: Seja $G$ grupo finito. Então pelo teorema 4.4 podemos considerar o grupo de unidades $\mathcal{U}_{1}(\mathbb{Z} G)$ como grupo linear finitamente gerado sobre $\mathbb{Z}$.

Sendo $\mathbb{Z}$ um anel comutativo, temos pelo teorema de Gruenberg (teorema 2.19) que o grupo de unidades $\mathcal{U}_{1}(\mathbb{Z} G)$ possui altura central finita, o que conclui a demonstração do teorema.

\subsection{Uma estimativa para a altura central de $\mathcal{U}_{1}(\mathbb{Z} G)$ utilizando a Conjectura do Normalizador}

Enunciamos, primeiramente, a Conjectura do Normalizador:

(NC) Seja $N_{\mathcal{U}}(G)$, o normalizador de $G$ em $\mathcal{U}_{1}(\mathbb{Z} G)$ então $N_{\mathcal{U}}(G)=G Z_{1}(\mathcal{U})$.

Essa Conjectura surgiu quando Jackowski e Marciniak estudavam os grupos de automorfismos de grupos topológicos que induziam a aplicação identidade em Cohomologia entre outros temas. Ou seja ela tem origem num problema topológico. Ao estudarem esses grupos de automorfismos de grupos topológicos e a sua relação com outros grupos de automorfismos mais conhecidos, eles obtiveram uma série de resultados que originaram a Conjectura do Normalizador.

Já vimos na seção anterior que a altura central de $\mathcal{U}$ é finita. É natural perguntar como podemos determinar um limitante superior para a altura central de $\mathcal{U}$. Nesse contexto, nos propomos a utilizar a Conjectura do Normalizador para obtermos uma estimativa para o limitante superior da altura central de $\mathcal{U}$, o qual será o nosso objetivo principal nesta seção.

\subsubsection{Notação e algumas definições}

Introduzimos algumas definições básicas e a notação que será empregada no restante do capítulo.

Definição 4.6. Seja $\pi$ um conjunto de inteiros primos. Então diremos que $G$ é um $\pi$-grupo se a ordem de $G$ for divisível somente por primos de $\pi$ e $G$ será um $\pi^{\prime}$-grupo se $|G|$ não for divisivel por nenhum primo $p$ em $\pi$. 
Definição 4.7. Diremos que $G$ é um grupo p-constrained se ele satisfaz a seguinte propriedade: Se P é um $S_{p}$-subgrupo de $O_{p, p^{\prime}}(G)$ então $C_{G}(P) \subseteq O_{p, p^{\prime}}(G)$.

\subsubsection{A estimativa para a altura central de $\mathcal{U}_{1}(\mathbb{Z} G)$}

Procuramos obter uma estimativa para o limitante superior para a altura central de $\mathcal{U}_{1}(\mathbb{Z} G)$. No caso em que o grupo $G$ é finito e não solúvel, usaremos um resultado de Gross para mostrar que nesse caso a altura central de $\mathcal{U}_{1}(\mathbb{Z} G)$ é no máximo 2. Por outro lado, ao estudarmos os grupos finitos $G$ tais que vale $N C$ veremos que a altura central de $\mathcal{U}_{1}(\mathbb{Z} G)$ também é no máximo 2. Logo, seria razoável conjecturarmos que a altura central de $\mathcal{U}_{1}(\mathbb{Z} G)$ é menor ou igual a 2 .

Lema 4.8. Seja $G$ um grupo finito e suponha que vale $N C$ para $G$. Então $Z_{n}(\mathcal{U}) \subseteq G Z_{1}(\mathcal{U})$ para todo $n \geq 1$.

Demonstração: Demonstraremos por indução em $n$. É suficiente mostrarmos que $Z_{n}(\mathcal{U}) \subseteq$ $N_{\mathcal{U}}(G)$, para todo $n \geq 2$.

O caso $n=1$ é imediato.

Suponhamos agora que a afirmação é válida para $n=k-1 \geq 3$ e iremos mostrar que ela é válida para $k$.

Sejam $u_{k} \in Z_{k}(\mathcal{U}), g \in G$. Então temos que $\left[u_{k}, g\right]=u_{k-1} \in Z_{k-1}(\mathcal{U})$. Pela hipótese de indução, temos que $Z_{k-1}(\mathcal{U}) \subseteq G Z_{1}(\mathcal{U})$, portanto, $u_{k-1}=g_{1} w$ para algum $g_{1} \in G, w \in Z_{1}(\mathcal{U})$. Segue daí que $\left[u_{k}, g\right]=u_{k-1}=g_{1} w$. Donde temos que $u_{k}{ }^{-1} g^{-1} u_{k}=g_{2} w$, onde $g_{2}=g_{1} g$. Elevando ambos os membros da igualdade a ordem do grupo $G$, obtemos que $w$ é uma unidade central de ordem finita. Segue pelo lema 1.16 que $c$ é uma unidade trivial. Logo $u_{k} \in N_{\mathcal{U}}(G)$. Como vale $N C$ para $G$ temos que $u_{k} \in G Z_{1}(\mathcal{U})$. Assim, $Z_{n}(\mathcal{U}) \subseteq G Z_{1}(\mathcal{U})$.

Teorema 4.9. (Gross, [25]) Sejam $G$ um grupo finito e p um inteiro primo. Se $C_{G}\left(O_{p}(G)\right) \leq$ $O_{p}(G)$ então todo automorfismo de $G$, que induza a identidade sobre um p-subgrupo de Sylow $P$, é interno.

Demonstração: Seja $\left.\rho\right|_{P}=i d$ um automorfismo nas hipóteses do teorema. Então temos que $\left.\rho\right|_{P}$ é a identidade em $O_{p}(G) \leq G$. Seja $g \in O_{p}(G)$. Segue que para todo $x \in G$ temos que $x^{-1} g x \in O_{p}(G)$. Logo $\rho(x)^{-1} g \rho(x)=\rho\left(x^{-1} g x\right)=x^{-1} g x$. O que implica que $x^{-1} \rho(x) \in$ $C_{G}\left(O_{p}(G)\right) \leq O_{p}(G)$, para todo $x \in G$. Segue daí que $x^{-1} \rho(x)$ é central em $O_{p}(G)$. Construímos o seguinte diagrama: 


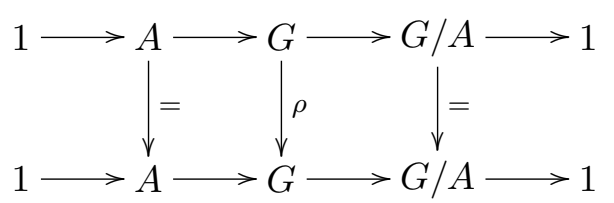

Consideremos agora o cociclo $\delta: G / A \longmapsto A, \delta([x])=x^{-1} \rho(x)$, onde $A=Z\left(O_{p}(G)\right)$. Como $A$ é um $p$-grupo, temos que a aplicação restrição res : $H^{1}(G / A, A) \longmapsto H^{1}(P / A, A)$ é injetiva (pois, $H^{1}(P / A, A)$ é um $p$-grupo). Mas por outro lado temos que res $[\delta]=0$, pois $\delta([x])=0$ para $x \in P$. Donde concluímos que $[\delta]=0$ e $\rho$ é um automorfismo interno.

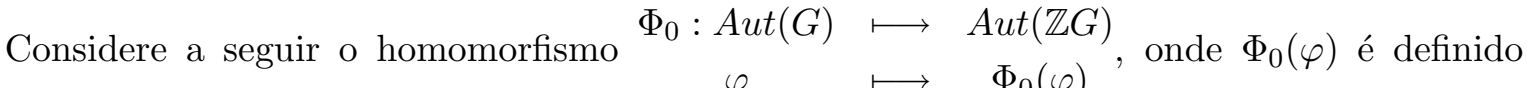
$\operatorname{como} \Phi_{0}(\varphi)(\alpha)=\Phi_{0}(\varphi)\left(\sum_{g \in G} \alpha_{g} g\right)=\sum_{g \in G} \alpha_{g} \varphi(g)$.

Como $\Phi_{0}(\operatorname{Inn}(G)) \subseteq \operatorname{Inn}(\operatorname{Aut}(\mathbb{Z} G))$ concluímos que $\Phi_{0}$ induz um homomorfismo $\Phi=$ $\Phi_{\mathbb{Z}}(G): \operatorname{Out}(G) \longmapsto \operatorname{Out}(\mathbb{Z} G)$. Observe que se $\varphi \in \operatorname{Out}(G)$ então $\varphi \in \operatorname{Ker}\left(\left(\Phi_{\mathbb{Z}}(G)\right)\right.$ se e somente se $\operatorname{Inn}(\operatorname{Aut}(\mathbb{Z} G))$, ou seja, $\varphi=\varphi_{u}$, para alguma unidade $u \in \mathcal{U}_{1}(\mathbb{Z} G)$; onde $\varphi_{u}(g)=u^{-1} g u, g \in G$. O que nos mostra que vale $N C$ se e somente se $\operatorname{Ker}\left(\left(\Phi_{\mathbb{Z}}(G)\right)=1\right.$.

Corolário 4.10. Sejam $G$ um grupo finito e p um inteiro primo. Se $C_{G}\left(O_{p}(G)\right) \leq O_{p}(G)$ então $\operatorname{Ker}\left(\Phi_{\mathbb{Z}}(G)\right)=1$. Em particular, temos que nessas condições vale NC para o grupo $G$.

Demonstração: Seja $\rho \in \operatorname{Ker}(\Phi)$. Então pelo lema 1.24 nós podemos supor que $\rho$ centraliza $P$, onde $P$ é um subgrupo de Sylow de $G$ fixado. Utilizando um raciocínio análogo ao que empregamos acima, concluímos que $\operatorname{Ker}\left(\Phi_{\mathbb{Z}}(G)\right)=1$.

Antes de demonstrarmos o próximo corolário do teorema de Gross, lembremos do seguinte:

Lema 4.11. ([17]) Seja $G$ um grupo $p$-constrained. Nesse caso se $\bar{G}=G / O_{p^{\prime}}(G)$. Então $C_{G}\left(O_{p}(\bar{G})\right) \subseteq O_{p}(\bar{G})$. Em particular, se $O_{p^{\prime}}(G)=1$ então $C_{G}\left(O_{p}(G)\right) \subseteq O_{p}(G)$.

Corolário 4.12. Seja $G$ um grupo finito não solúvel. Então $Z_{2}(\mathcal{U})=Z_{1}(\mathcal{U})$.

Demonstração: Suponha que $G$ seja um contraexemplo minimal e seja $F$ o subgrupo de Fitting de $G$. Afirmamos inicialmente que podemos supor que $F$ seja um $p$-grupo para algum primo $p$. Sejam $u \in Z_{2}(\mathcal{U}), g \in G$. Sabemos que $[u, g]=\rho(g)$ para algum $\rho(g) \in Z_{1}(G)$. Conseqüentemente temos que $u^{-1} g u=\rho(g) g$. Se $\rho(g)$ não for trivial segue que $Z(G)$ é não trivial, o que implica que $F$ não é trivial, visto que $Z(G) \subseteq F$.

Suponha que $p$ e $q$ sejam primos distintos que dividem a ordem de $F$ e que $Z_{2}(\mathcal{U}) \nsupseteq Z_{1}(\mathcal{U})$. Seja $u \in Z_{2}(\mathcal{U}) \backslash Z_{1}(\mathcal{U})$. Então existe $g \in G$ tal que $[u, g] \neq 1$. 
Como $F$ é nilpotente (pois o grupo $G$ é finito), temos então que $S y l_{p}(F) \triangleleft G$ e $S y l_{q}(F) \triangleleft G$.

Considere o epimorfismo canônico $\pi: \mathbb{Z} G \longmapsto \mathbb{Z}\left(G / S y l_{p}(F)\right)$. Aplicando $\pi$ a $[u, g]$ obtemos que $\overline{[u, g]}=\overline{1}$ visto $G$ ser um contraexemplo minimal. Concluímos daí que $[u, g] \in S_{y} l_{p}(F)$. Com raciocínio análogo tomando $\pi_{1}: \mathbb{Z} G \longmapsto \mathbb{Z}\left(G / S y l_{q}(F)\right)$ obtemos que $[u, g] \in \operatorname{Syl}_{q}(F)$. Conseqüentemente temos que $[u, g] \in \operatorname{Syl}_{p}(F) \cap S y l_{q}(F)=(1)$. Contradição. Logo podemos supor que $F$ seja um $p$-grupo para algum primo $p$ e logo, $F=O_{p}(G)$.

Afirmamos que $O_{p^{\prime}}(G)=1$.

Suponha que $O_{p^{\prime}}(G)$ fosse diferente de 1. Então, empregando o mesmo raciocínio acima e utilizando o fato que $O_{p}(G) \cap O_{p^{\prime}}(G)=1$, obteríamos uma contradição. Donde $O_{p^{\prime}}(G)=1$. Logo, pelo lema 4.11 segue que $C_{G}(F)=C_{G}\left(O_{p}(G)\right) \subseteq O_{p}(G)$ e portanto, $G$ satisfaz as hipóteses do teorema de Gross.

Assim, se $u \in Z_{2}(\mathcal{U}) \backslash Z_{1}(\mathcal{U})$. Então, pelo corolário 4.10 (do teorema de Gross) segue que $u=g w$ para algum $g \in G$ e algum $w \in Z_{1}(\mathcal{U})$, onde $g \notin Z_{1}(\mathcal{U})$. Como $g$ é um elemento de torção do hipercentro de $\mathcal{U}$ segue pelo teorema 3.21 que $G$ é um 2 -grupo Hamiltoniano ou $G$ é um $Q^{*}$-grupo, os quais são ambos grupos solúveis, contradição. Logo $u \in Z_{1}(\mathcal{U})$, o que implica que $Z_{2}(\mathcal{U})=Z_{1}(\mathcal{U})$.

Finalmente, podemos obter uma estimativa para a altura central de $\mathcal{U}_{1}(\mathbb{Z} G)$ através do seguinte lema:

Lema 4.13. Seja $G$ um grupo finito e suponha que vale $N C$ para $G$. Então a altura central de $\mathcal{U}_{1}(\mathbb{Z} G)$ é no máximo 2.

Demonstração: Suponha que $u \in \widetilde{Z}(\mathcal{U}) \backslash Z_{1}(\mathcal{U})$, onde $\widetilde{Z}(\mathcal{U})$ denota o hipercentro de $\mathcal{U}_{1}(\mathbb{Z} G)$. Como a altura central de $\mathcal{U}_{1}(\mathbb{Z} G)$ é finita (pelo lema 4.5) temos que $u \in Z_{n}(\mathcal{U})$ para algum $n$. Como vale $N C$ para $G$ segue pelo lema 4.8 que $u=g w$ para algum $g \in G$ e algum $w \in Z_{1}(\mathcal{U})$. Sendo $g$ um elemento de torçao de $\widetilde{Z}(\mathcal{U}) \backslash Z(G)$ segue pelo teorema 3.21 que $g \in Z_{2}(\mathcal{U}) \backslash Z(G)$, o que implica que $u \in Z_{2}(\mathcal{U})$. Conseqüentemos temos que $\widetilde{Z}(\mathcal{U})=Z_{2}(\mathcal{U})$.

Donde podemos concluir que se vale $N C$ então em qualquer caso a altura central de $\mathcal{U}_{1}(\mathbb{Z} G)$ é no máximo 2 .

\subsection{A altura central de $\mathcal{U}_{1}(\mathbb{Z} G)$ é no máximo 2}

Na seção anterior, notamos que em vários casos a altura central de $\mathcal{U}_{1}(\mathbb{Z} G)$ seria no máximo 2. Então é razoável conjecturarmos que a altura central de $\mathcal{U}_{1}(\mathbb{Z} G)$ é no máximo 2. 
Nesta seção, apresentaremos o resultado de Arora, Hales e Passi de que a altura central de $\mathcal{U}_{1}(\mathbb{Z} G)$ de um grupo finito $G$ qualquer é no máximo 2.

Lema 4.14. ([7]) Sejam $G$ um grupo finito e K um corpo de característica zero. Todo elemento $\alpha \in K G$ possui uma única decomposição de Jordan $\alpha=\alpha_{s}+\alpha_{n}, \alpha_{s}, \alpha_{n} \in K G, \alpha_{s}$ semisimples ( ou seja, o seu polinômio minimal sobre $K$ não possui raízes múltiplas no fecho algébrico $\bar{K}$ de $K), \alpha_{n}$ nilpotente e $\alpha_{s} \alpha_{n}=\alpha_{n} \alpha_{s}$.

Lema 4.15. ([7]) Se $\alpha \in \mathcal{U}_{1}(K G)$ então temos uma única decomposição de Jordan multiplicativa $\alpha=\beta \delta, \beta, \delta \in \mathcal{U}_{1}(K G), \beta$ semisimples e $\delta$ unipotente. De fato, $\beta=\alpha_{s}$ e $\delta=1+\alpha_{s}{ }^{-1} \alpha_{n}$.

Nas condições do Teorema 3.21 segue que:

Corolário 4.16. Todas as unidades hipercentrais de torção de $\mathcal{U}=\mathcal{U}_{1}(\mathbb{Z} G)$ estão contidas em $\pm Z_{2}(G)$.

Proposição 4.17. Sejam $G$ um grupo finito e e o expoente de $Z_{1}(G)$. Então:

(1) $\left[Z_{2}(\mathcal{U}), \mathcal{U}\right] \leq Z_{1}(G)$;

(2) $Z_{n+1}^{e}(\mathcal{U}) \leq Z_{n}(\mathcal{U})$, para todo $n \geq 1$;

(3) Toda unidade hipercentral em $\mathcal{U}$ é semisimples e comuta com todo elemento unipotente de $\mathcal{U}$.

Demonstração: Mostraremos inicialmente que $\left[Z_{2}(\mathcal{U}), G\right] \leq Z_{1}(G)$.

Sejam $u_{2} \in Z_{2}(\mathcal{U}), g \in G$. Então temos que $\left[u_{2}, g\right]=c$, para algum $c \in Z_{1}(\mathcal{U})$. Elevando ambos os lados da igualdade a $|G|$ e utilizando a proposição 1.33 obtemos que $1=\left[u_{2}, g\right]^{|G|}=$ $\left[u_{2}, g^{|G|}\right]=c^{|G|}$. Donde concluímos que $c$ é uma unidade central de ordem finita. Segue pelo lema 1.16 que $c$ é uma unidade trivial. Demonstrando a afirmação.

Seja agora $e$ o expoente de $Z_{1}(G)$. Como $\left[Z_{2}(\mathcal{U}), G\right] \leq Z_{1}(G)$, obtemos utilizando a proposição 1.33 que $\left[Z_{2}^{e}(\mathcal{U}), G\right]=1$, donde concluímos que $Z_{2}^{e}(\mathcal{U}) \leq Z_{1}(\mathcal{U})$. Segue desse fato que $\left[Z_{2}(\mathcal{U}), \mathcal{U}\right] \leq Z_{1}(G)$ e por indução em $n$ que $Z_{n+1}^{e}(\mathcal{U}) \leq Z_{n}(\mathcal{U})$, para todo $n \geq 1$.

De fato, seja $u_{2} \in Z_{2}(\mathcal{U}), u \in \mathcal{U}$.Então $\left[u_{2}, u\right]=c_{1}$, para algum $c_{1} \in Z_{1}(\mathcal{U})$. Utilizando o lema 1.33 temos que $c_{1}^{e}=\left[u_{2}, u\right]^{e}=\left[u_{2}^{e}, u\right]=1$. Logo $c_{1}$ é uma unidade central de ordem finita. Segue pelo lema 1.16 que $c_{1}$ é uma unidade trivial. Mostrando que $\left[Z_{2}(\mathcal{U}), \mathcal{U}\right] \leq Z_{1}(G)$.

A seguir mostraremos que $Z_{n+1}^{e}(\mathcal{U}) \leq Z_{n}(\mathcal{U})$, para todo $n \geq 1$.

Já demonstramos a afirmação para $n=1$.

Suponhamos que a afirmação é válida para $n=k-1 \geq 2$. Devemos mostrar que ela é válida para $n=k$. 
Sejam $u_{k+1} \in Z_{k+1}(\mathcal{U}), u \in \mathcal{U}$. Então temos que $\left[u_{k+1}, u\right]=u_{k} \in Z_{k}(\mathcal{U})$.

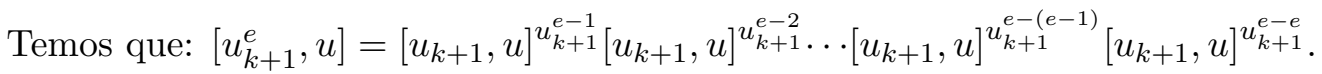

Desenvolvendo o primeiro fator da expressão acima obtemos:

$\left[u_{k+1}, u\right]^{u_{k+1}^{e-1}}=u_{k+1}^{-(e-1)}\left[u_{k+1}, u\right] u_{k+1}^{e-1}=u_{k+1}^{-(e-1)}\left[u_{k+1}, u\right] u_{k+1}^{e-1} u_{k}^{-1} u_{k}=\left[u_{k+1}^{e-1}, u_{k}^{-1}\right] u_{k}$.

Conseqüentemente temos que:

$\left[u_{k+1}^{e}, u\right]=\underbrace{\left[u_{k+1}^{e-1}, u_{k}^{-1}\right]}_{\in Z_{k-1}(\mathcal{U})} u_{k} \underbrace{\left[u_{k+1}^{e-2}, u_{k}^{-1}\right]}_{\in Z_{k-1}(\mathcal{U})} u_{k} \cdots \underbrace{\left[u_{k+1}, u_{k}^{-1}\right]}_{\in Z_{k-1}(\mathcal{U})} u_{k} u_{k}$.

Considere o epimorfismo $\psi: Z_{k+1}(\mathcal{U}) \longmapsto Z_{k+1}(\mathcal{U}) / Z_{k}(\mathcal{U})$. Temos então que:

$\psi\left(\left[u_{k+1}^{e}, u\right]\right)=\overline{1} \overline{u_{k}^{-1}} \overline{1} \overline{u_{k}^{-1}} \cdots \overline{1} \overline{u_{k}^{-1} u_{k}^{-1}}=\overline{u_{k}^{e}}$. Segue da hipótese de indução que $Z_{k}^{e}(\mathcal{U}) \leq$ $Z_{k-1}(\mathcal{U})$, o que implica que $\left[u_{k+1}^{e}, u\right] \in Z_{k-1}(\mathcal{U})$, concluindo a demonstração da afirmação.

Afirmamos que todo elemento central de $\mathcal{U}$ é semisimples.

Para observar este fato, note inicialmente que qualquer representação complexa irredutível de $G$ leva qualquer elemento central de $\mathcal{U}$ numa matriz escalar. Por outro lado, sabemos que $Z_{n+1}^{e}(\mathcal{U}) \leq Z_{n}(\mathcal{U})$, para todo $n \geq 1$, o que implica diretamente que o quociente $\widetilde{Z}(\mathcal{U}) / Z_{1}(\mathcal{U})$ é periódico.

Observando agora que se $\alpha \in \mathcal{U}$, e uma potência de $\alpha$ é semisimples então $\alpha$ é semisimples, podemos concluir utilizando os fatos acima que todo elemento central de $\mathcal{U}$ é semisimples.

Afirmamos que se $\alpha \in \mathcal{U}$ e uma potência de $\alpha$ é semisimples então $\alpha$ é semisimples.

De fato, se $\alpha \in \mathcal{U}$ então pelo lema $4.15, \alpha$ possui uma única decomposição de Jordan multiplicativa, ou seja, podemos expressar $\alpha$ na forma $\alpha=\beta \delta, \beta \delta=\delta \beta$ com $\beta$ semisimples e $\delta$ unipotente. Se $\alpha^{k}$ é semisimples, para algum $k \in \mathbb{Z}$ então como $\beta$ e $\gamma$ comutam, temos que $\alpha^{k}=\beta^{k} \delta^{k}$, donde concluímos que $\delta^{k}$ é um elemento semisimples. Sendo $\delta$ um elemento semisimples e nilpotente concluímos que $\delta$ é igual a $1_{|G|}$, a matriz identidade de ordem $|G|$. Conseqüentemente, $\alpha=\beta$ e portanto, $\alpha$ é semisimples, provando a afirmação.

A seguir utilizando a unicidade da decomposição multiplicativa de Jordan e indução em $n$, obtemos facilmente que todo elemento de $Z_{n}(\mathcal{U}), n \geq 1$, comuta com todo elemento unipotente de $\mathcal{U}$. Concluindo a demonstração da proposição.

Corolário 4.18. Se $Z_{1}(G)=(1)$ então $Z_{1}(\mathcal{U})=Z_{2}(\mathcal{U})$. 
A seguir enunciaremos alguns lemas:

Lema 4.19. Seja $G$ um $Q^{*}$-grupo. Então $\left[Z_{3}(\mathcal{U}), \mathcal{U}\right] \subseteq T$.

Demonstração: Sejam $u_{3} \in Z_{3}(\mathcal{U})$ e $u \in \mathcal{U}$ e considerando a identidade $\left[u_{3}, u\right]^{2}=\left[u_{3},\left[u_{3}, u\right]\right]^{-1}\left[u_{3}^{2}, u\right]$ nós obtemos pela proposição $4.17(1)$ e $(2)$ que $\left[u_{3}, u\right]^{2} \in Z_{1}(G)$. Conseqüentemente obtemos que $\left[Z_{3}(\mathcal{U}), \mathcal{U}\right] \subseteq T$.

Lema 4.20. Seja $G$ um $Q^{*}$-grupo. Então: $\left[Z_{3}(\mathcal{U}), G\right] \subseteq\left\langle a^{2}\right\rangle$.

Demonstração: Seja $H$ o subgrupo abeliano de índice 2 dado na definição de $Q^{*}$-grupo. Observamos inicialmente que qualquer elemento $g \in G \backslash H$ possui ordem 4 .

Sejam $x \in Z_{3}(\mathcal{U}), g \in G$.

Observamos inicialmente que para qualquer $h \in H$ para o qual $h^{-1} g h \in\langle g\rangle$ então $h^{2}=1$ ou $(a h)^{2}=1$. Para verificar este fato observe que:

$g h^{2}=g g^{-1} h^{-1} g h=h^{-1} g h \in\langle g\rangle$. Logo, $h^{2} \in\langle g\rangle$.

Se $h^{2}=g$ ou $h^{2}=g^{3}$ então $[g, h]=1$. Absurdo, pois $g$ não é um elemento central de G. Caso contrário, $h^{2}=1$ ou $h^{2}=g^{2}=a^{2}$. No caso em que $h^{2}=g^{2}=a^{2}$ obtemos que $(a h)^{2}=a h a h=a^{2} h^{2}=a^{2} a^{2}=a^{4}=1$, pois pelo teorema $3.20,\langle a\rangle \subseteq H$.

Conseqüentemente obtemos que $h \in \mathcal{Z}(G)$ ou $a h \in \mathcal{Z}(G)$.

Afirmamos agora que $[x, h] \equiv[x, a h]\left(\bmod \left\langle a^{2}\right\rangle\right)$.

Como $\langle a\rangle \triangleleft \mathcal{U}$ pela demonstração do teorema $3.21\left((2) \Rightarrow(1)\right.$ ). Então $a \in Z_{2}(\mathcal{U})$. (Conforme a demonstração do teorema 3.21). A seguir, utilizando a proposição 1.32(ii), temos que:

$$
[x, a h]=[x, h] \underbrace{[x, a]^{h}}_{\in Z_{1}(G)}=[x, h][x, a] \Rightarrow[x, a h] \equiv[x, h] \quad\left(\bmod \left\langle a^{2}\right\rangle\right) .
$$

O que por sua vez implica que $[x, h] \in\left\langle a^{2}\right\rangle$.

Suponhamos agora que $h \in H, h^{-1} g h \notin\langle g\rangle$.

Então a unidade $1+\widehat{g} h(1-g)$ é um elemento unipotente não-idêntico de $\mathcal{U}$. ( Um elemento $\delta$ se diz unipotente se $1-\delta$ for um elemento nilpotente).

Pela proposição 4.17(3) temos que:

$$
\widehat{g} h(1-g) x=x \widehat{g} h(1-g)
$$

o que implica que:

$$
\widehat{g} h x-\widehat{g} h g x=x \widehat{g} h-x \widehat{g} h g .
$$


Pelo lema 4.19 segue que $x^{-1} g x \in G$ e $x^{-1} h x \in G$, pois $\left[x, g^{-1}\right] \in G$ e $\left[x, h^{-1}\right] \in G$.

Como $h \in H, h^{-1} g h \notin\langle g\rangle$, nós concluímos de 4.1 que:

$$
\begin{gathered}
\widehat{g} h x=x \widehat{g} h, \text { e portanto, } \\
x^{-1} \widehat{g} h x=\widehat{g} h .
\end{gathered}
$$

Conseqüentemente,

$$
x^{-1} h x \in\langle g\rangle h .
$$

O qual mostra que: $\left[x, h^{-1}\right] \in\langle g\rangle \cap T=\left\langle a^{2}\right\rangle$.

Portanto, $[x, h] \in\left\langle a^{2}\right\rangle$.

Além disso, 4.1 também implica que: $x^{-1} \widehat{g} x=\widehat{g}$, pois de 4.1 e do lema 4.19 obtemos que $x^{-1} \widehat{g} h x=x^{-1} \widehat{g} x \underbrace{x^{-1} h x h^{-1}}_{\in\langle g\rangle}=x^{-1} \widehat{g} x g^{i}=\widehat{g}$ para algum $i \Rightarrow x^{-1} \widehat{g} x=g^{-i} \widehat{g}=\widehat{g}$.

Logo, $x^{-1} \widehat{g} x \in\langle g\rangle$, donde concluímos que: $[x, g] \in\left\langle a^{2}\right\rangle$.

Observe que como $G$ não é um 2-grupo Hamiltoniano então existe um $h \in H$ tal que $h^{-1} g h \notin\left\langle a^{2}\right\rangle$. Concluindo a demonstração do lema.

Uma conseqüência imediata do lema é o:

Corolário 4.21. Seja $G$ um $Q^{*}$-grupo então $\left[Z_{3}^{2}(\mathcal{U}), G\right]=1$, donde $Z_{3}^{2}(\mathcal{U}) \subseteq Z_{1}(\mathcal{U})$.

Podemos finalmente enunciar o resultado principal da seção e um dos principais resultados da nossa dissertação.

Teorema 4.22. (Arora, Hales, Passi) Seja $G$ um grupo finito. Então a altura central do grupo de unidades $\mathcal{U}_{1}(\mathbb{Z} G)$ é no máximo 2.

Demonstração: De acordo com o teorema 3.21 temos de considerar três casos:

(1) Suponhamos que $G$ seja um 2-grupo Hamiltoniano. Então pela proposição 1.30 e pelo lema $1.19, \mathcal{U}= \pm G= \pm T=Z_{2}(\mathcal{U})$.

(2) Suponhamos que $T$ seja um subgrupo central de $\mathcal{U}$. Nesse caso pela proposição 4.17(1) concluímos que $Z_{1}(\mathcal{U} / T)=Z_{2}(\mathcal{U}) / T$ (o qual é livre de torção). Segue pela proposição 2.2 que os fatores da série central superior de $\mathcal{U} / T$ são livres de torção, donde concluímos que o quociente $Z_{3}(\mathcal{U}) / Z_{2}(\mathcal{U})$ é livre de torção. Finalmente pela proposição $4.17(2)$ decorre que $Z_{2}(\mathcal{U})=Z_{3}(\mathcal{U})$ 
(3) Suponhamos agora que $T$ é abeliano, mas não seja um subgrupo central. Nesse caso, $G=\langle H, b\rangle$ é um grupo do tipo (3) no teorema 3.21, ou seja, $G$ é um $Q^{*}$-grupo, portanto, $T=\langle a\rangle \oplus E=Z_{2}(\mathcal{U}) \cap Z_{2}(G)$.

Suponhamos por absurdo que exista $x \in Z_{3}(\mathcal{U}) \backslash Z_{2}(\mathcal{U})$. Então para algum $u \in \mathcal{U},[x, u]=$ $t \in T$ é um elemento de ordem 4. Afirmamos que $t \in G^{\prime}$. Para observarmos esse fato, considere a projeção natural $\varphi: \mathbb{Z} G \longmapsto \mathbb{Z}\left(G / G^{\prime}\right)$, aplicando $\varphi$ a $t$ obtemos que $\varphi(t)=\bar{t}$, decorre daí que: $[\bar{x}, \bar{u}]=\bar{t}=\overline{1}$, pois $\mathbb{Z}\left(G / G^{\prime}\right)$ é um anel de grupo comutativo. Portanto, $t-1 \in \triangle\left(G^{\prime}\right) \mathbb{Z} G$ o que implica que $t \in G^{\prime}$. Pelo lema $4.20[x, h] \in\left\langle a^{2}\right\rangle \subseteq Z_{1}(\mathcal{U})$ para todo $h \in H, \operatorname{logo}[x, h]^{2}=1$. Portanto para algum $h \in H$ temos que: $[x, t]=\left[x, h^{2}\right]=$ $[x, h] h[x, h] h^{-1}=[x, h]^{2}=1$. Conseqüentemente $\left[x^{2}, u\right]=x[x, u] x^{-1}[x, u]=[x, t] t^{2}=t^{2} \neq$ 1. Mas pelo corolário 4.21 nós obtemos que $\left[x^{2}, u\right]=1$. Contradição. $\operatorname{Logo} Z_{2}(\mathcal{U})=Z_{3}(\mathcal{U})$.

Corolário 4.23. Se todas as unidades centrais em $\mathcal{U}_{1}(\mathbb{Z} G)$ são triviais então todas as unidades hipercentrais são triviais também.

Demonstração: Pelo teorema 4.22 e utilizando o fato que $\left[Z_{2}(\mathcal{U}), G\right] \leq Z_{1}(G)$ pelo lema $4.17(1)$ temos que $\widetilde{Z}(\mathcal{U}) \subseteq N_{\mathcal{U}}(G)$. Seja $u \in \widetilde{Z}(\mathcal{U})$. Então temos que $u \in N_{\mathcal{U}}(G)$. Além disso, como $\widetilde{Z}(\mathcal{U}) / Z_{1}(\mathcal{U})$ é periódico concluímos que existe um $n=n(u)$ tal que $u^{n} \in G$. Como $u \in N_{\mathcal{U}}(G)$ temos pelo lema 1.23 que $u^{*} u=u u^{*}$. Além disso, como $u^{n}=g$ para algum $g \in G$ temos que $\left(u^{*} u\right)^{n}=\left(u^{*}\right)^{n}(u)^{n}=g^{-1} g=1$. Segue daí que $u^{*} u$ é uma unidade central de ordem finita. Conseqüentemente, pelo lema 1.16 concluímos que $u^{*} u \in Z_{1}(G)$. Afirmamos que $u \in G$.

De fato, seja $u=\sum_{g \in G} u_{g} g$. Então temos que $u^{*} u(1)=\sum_{g \in G} u_{g}^{2} \neq 0$. Donde temos pelo lema 1.15 que $u^{*} u=1$ e conseqüentemente pelo lema 1.17 temos que $u \in G$.

Relembramos do teorema de Ritter-Sehgal:

Teorema 4.24. (Ritter-Sehgal) Seja $G$ um grupo finito. Todas as unidades centrais de $\mathbb{Z} G$ são triviais se e somente se para todo $x \in G$ e todo número natural $j$ relativamente primo com $|G|$, nós tivermos $x^{j} \sim x$ ou $x^{j} \sim x^{-1}$, onde $\sim$ representa a conjugação em $G$.

Como uma conseqüência do teorema de Ritter-Sehgal temos o seguinte corolário:

Corolário 4.25. Seja $G$ um grupo finito. Todas as unidades hipercentrais de $\mathbb{Z} G$ são triviais se e somente se para todo $x \in G$ e todo número natural $j$ relativamente primo a $|G|, x^{j}$ é conjugado a $x$ ou $a x^{-1}$. 


\subsection{Uma caracterização do segundo centro de $\mathcal{U}_{1}(\mathbb{Z} G)$}

Pelos resultados da seção anterior, concluímos que a altura central de $\mathcal{U}_{1}(\mathbb{Z} G)$ de um grupo finito $G$ é sempre menor ou igual a 2 . Com base neste resultado nosso objetivo nesta seção será o de procurarmos uma caracterização para o hipercentro de $\mathcal{U}$ utilizando os resultados do capítulo 3, o teorema de Bovdi.

Com esse objetivo em vista, nas duas subseções seguintes apresentamos o teorema de Blackburn e a demonstração de que a Conjectura do Normalizador é satisfeita para os grupos finitos $G$ tais que $R(G) \neq 1$, onde $R(G)$ indica a intersecção dos subgrupos não-normais de $G$.

Após desenvolvermos estes pré-requisitos, finalmente caracterizamos o hipercentro de $\mathcal{U} \mathrm{em}$ função do centro de $\mathcal{U}$ e do subgrupo de torção do hipercentro de $\mathcal{U}$. Esse resultado será o objetivo principal desta seção e será apresentado na última subseção deste capítulo.

Após obtermos esta caracterização do hipercentro de $\mathcal{U}$ concluíremos que a Conjectura do Normalizador é satisfeita para as unidades hipercentrais; isto é, $\widetilde{Z}(\mathcal{U}) \subseteq G Z_{1}(\mathcal{U})$.

Os resultados desta seção estão relacionados nas referências [3], [6], [20] e [23].

\subsubsection{O teorema de classificação de $p$-grupos de Blackburn}

A seguir $R(G)$ indicará a intersecção dos subgrupos não-normais de $G$.

Apresentamos sem demonstração um teorema bem conhecido de Blackburn que utilizaremos nas subseções seguintes:

Teorema 4.26. (Blackburn) Seja $G$ um grupo finito, $R(G)$ não trivial, $G$ não Dedekind e tal que $G$ não possui ordem potência de um primo. Então ocorre uma das seguintes situações:

(a) G possui um subgrupo abeliano $A$ de expoente $k p^{n}$ onde $n \geq 1, p$ primo e $(k, p)=1$. $G / A$ é cíclico de ordem $p^{r}$ e se Au gera $G / A$, u pode ser escolhido de maneira que $u^{p^{r}}$ possua ordem $p^{n}$. Existe um inteiro $\xi \equiv 1\left(p^{n}\right)$ tal que $x^{u}=x^{\xi}$ para todo a em $A$.

(b) Gé o produto direto de um grupo abeliano de ordem ímpar e um dos seguintes grupos:

(1) O produto direto de um grupo quatérnion de ordem 8, um grupo cíclico de ordem 4 e um 2-grupo abeliano elementar.

(2) O produto direto de dois grupos quatérnions de ordem 8 e um 2-grupo abeliano elementar.

(c) $G$ possui um subgrupo $H$ do tipo descrito em $(a) \operatorname{com} p=2, r=1$. $H$ é de índice 2 e se $G$ é gerado por $H$ e $t$, então $t$ pode ser escolhido de maneira que $u^{t}=u^{-1}, t^{2}=u^{2^{n}} e$ $x^{t}=x^{\eta}$ para algum $\eta \equiv-1\left(2^{n}\right)$. 
(d) G possui um subgrupo abeliano $A$ de indice 2 . G é gerado por $A$ e $t$ onde $t^{2}$ é um elemento de $A$ de ordem 2. Se $x$ é um elemento de $A, x^{t}=x^{\zeta}$ para algum $\zeta \equiv-1\left(2^{n}\right)$.

(e) $G$ é o produto direto de $H$, um grupo quatérnion de ordem 8, e um 2-grupo abeliano elementar, onde $H$ possui ordem ímpar e é do tipo descrito em (a).

\subsubsection{A Conjectura do Normalizador para grupos G tais que $R(G)$ não é trivial}

Iremos denotar por $N C$ a Conjectura do Normalizador. Nosso objetivo nesta subseção será o de apresentarmos o seguinte resultado:

Teorema 4.27. Seja $G$ um grupo finito. Se $R(G)$ não é trivial então vale $N C$ para $G$.

Com o objetivo acima apresentado, enunciamos os bem conhecidos teoremas de Coleman e de Jacowski-Marciniak e provamos alguns teoremas.

Teorema 4.28. (Coleman) Seja $G$ um grupo nilpotente finito. Então vale NC para $G$.

Teorema 4.29 (Jacowski-Marciniak). Se G é um grupo de ordem ímpar. Então vale $N C$ para $G$.

Teorema 4.30. Seja $G=\langle H, g\rangle$ onde $H$ é um subgrupo abeliano de indice 2. Então vale $N C$ para $G$.

Demonstração: Basta mostrarmos que $\mathcal{N}_{\mathcal{U}}(G) \subseteq G Z_{1}(\mathcal{U})$. Seja $u \in \mathcal{N}_{\mathcal{U}}(G)$, podemos expressar $u$ na forma $u=\alpha_{1}+\alpha_{2} g$, onde $\alpha_{1}$ e $\alpha_{2} \in \mathbb{Z} H$. Observamos inicialmente que como $H$ é um subgrupo de índice 2 , o anel de grupo $\mathbb{Z}(G / H)$ possui somente unidades triviais. Aplicando o epimorfismo canônico $\varphi: \mathbb{Z} G \longmapsto \mathbb{Z}(G / H)$, temos que: $\bar{u}=\epsilon\left(\alpha_{1}\right)+\epsilon\left(\alpha_{2}\right) \bar{g}$, onde $\epsilon$ denota o homomorfismo de aumento. Como $\bar{u}$ deve ser uma unidade trivial de $\mathbb{Z}(G / H)$, concluímos que $\epsilon\left(\alpha_{1}\right)= \pm 1$ e $\epsilon\left(\alpha_{2}\right)=0$ ou $\epsilon\left(\alpha_{1}\right)=0$ e $\epsilon\left(\alpha_{2}\right)= \pm 1$. Multiplicando se necessário por $\pm g$ ou -1 podemos supor que $\epsilon\left(\alpha_{1}\right)=1$ e $\epsilon\left(\alpha_{2}\right)=0$.

Afirmamos inicialmente que $[u, h] \in H$ para todo $h \in H$.

Como $H \triangleleft G$ e $u \in \mathcal{N}_{\mathcal{U}}(G)$, segue que $t=[u, h] \in G$. Aplicando o epimorfismo canônico $\varphi: \mathbb{Z} G \longmapsto \mathbb{Z}(G / H)$ obtemos que $\bar{t}=\overline{1}$. O que nos mostra que $[u, h] \in H$ para todo $h \in H$.

Segue do fato anterior que existe algum $h_{0} \in H$ tal que $[u, h]=h_{0}$, ou seja, $h u=u h h_{0}$. Conseqüentemente temos que $h \alpha_{1}=\alpha_{1} h h_{0}$, donde concluímos que $\alpha_{1}\left(1-h_{0}\right)=0$.

Afirmamos que $h_{0}$ possui ordem finita.

De fato, como $\alpha_{1}\left(1-h_{0}\right)=0$ temos que $\alpha_{1}=\alpha_{1} h_{0}$ o que implica que $\alpha_{1}=\alpha_{1} h_{0}^{k}$, para todo $k \in \mathbb{N}$. Seja $\alpha_{1}=\sum_{g \in G} \alpha_{g} g$. Então teríamos que $\alpha_{1}=\sum_{g \in G} \alpha_{g} g=\sum_{g \in G} \alpha_{g} g h_{0}^{k}$, para todo 
$k \in \mathbb{N}$. Se $h_{0}$ tivesse ordem infinita deveríamos ter infinitas possibilidades para os elementos do suporte no lado esquerdo da igualdade. Contradição com o fato do suporte de $\alpha_{1}$ ser finito.

Afirmamos que $h_{0}=1$ e que $[u, h]=1$ para todo $h \in H$.

Já observamos que $h_{0}$ possui ordem finita. Suponha agora que $\alpha_{1}\left(1-h_{0}\right)=0$, ou seja, temos que $\alpha_{1}=\alpha_{1} h_{0}$, o que por sua vez implica que $\alpha_{1}=\alpha_{1} h_{0}^{k}$, para todo inteiro $k$. Sejam $X=\operatorname{supp}\left(\alpha_{1}\right)$ e $\alpha_{1}=\sum_{g \in G} \alpha_{g} g$. Então $\alpha_{1}=\sum_{g \in G} \alpha_{g} g=\sum_{g \in G} \alpha_{g} g h_{0}$. A seguir, considere a ação de $\left\langle h_{0}\right\rangle$ sobre $X$, dada por:

$$
\begin{array}{ccc}
X \times\left\langle h_{0}\right\rangle & \longmapsto & X \\
\left(g, h_{0}^{k}\right) & \longmapsto & g h_{0}^{k}
\end{array}
$$

Observe a seguir que se $x \in X$ então a órbita $\mathcal{O}_{x}$ de $x$ satisfaz $\left|\mathcal{O}_{x}\right|=o\left(h_{0}\right)$ visto que $\mathcal{O}_{x}=\left\{x, x h_{0}, \cdots, x h_{0}^{o\left(h_{0}\right)-1}\right\}$. Além disso, temos que $\alpha_{g}=\alpha_{x}$, para todo $g \in \mathcal{O}_{x}$.

A seguir expresse $X$ como $X=\cup \dot{\cup} \mathcal{O}_{x_{j}}$, união disjunta de órbitas. Então temos que $\alpha_{1}=$ $\sum_{j} \sum_{g \in \mathcal{O}_{x_{j}}} \alpha_{g} g$. Segue daí que:

$1=\epsilon\left(\alpha_{1}\right)=\sum_{j} \alpha_{x_{j}}\left|\mathcal{O}_{x_{j}}\right|=\left(\sum_{j} \alpha_{x_{j}}\right) o\left(h_{0}\right)=m\left(o\left(h_{0}\right)\right)$, para algum $m \in \mathbb{Z}$. O que por sua vez implica que $o\left(h_{0}\right)=1$ e portanto, $h_{0}=1$.

Como $h_{0}=1$ segue que $[u, h]=1$ para todo $h \in H$.

Observamos agora que $[u, g] \in G^{\prime}$.

De fato, tomando o epimorfismo canônico $\pi: \mathbb{Z} G \longmapsto \mathbb{Z}\left(G / G^{\prime}\right)$, segue imediatamente que $\overline{[u, g]}=\overline{1}$, visto $\mathbb{Z}\left(G / G^{\prime}\right)$ ser um anel de grupo comutativo. Concluímos daí que $[u, g] \in G^{\prime}$, para todo $g \in G$.

A seguir, como $H$ é um subgrupo de índice 2, concluímos que $G^{\prime}$ é formado por todos os comutadores da forma $[g, h], h \in H$.

Portanto temos que $[u, g]=[g, h]$ para algum $h \in H$. O que por sua vez implica que $u^{-1} g^{-1} u=h^{-1} g h$, donde tiramos que $\left[u h^{-1}, g\right]=1$.

Se mostrarmos que $u h^{-1}$ é central em $\mathcal{U}_{1}(\mathbb{Z} G)$ então a tese segue diretamente. De fato, seja $h^{\prime} \in H$, então temos que $\left[u h^{-1}, h^{\prime}\right]=h\left[u, h^{\prime}\right]\left(h^{\prime}\right)^{-1} h^{-1} h^{\prime}=1$, visto que $\left[u, h^{\prime}\right]=1$ como vimos anteriormente. Donde concluímos que $u \in G Z_{1}(\mathcal{U})$. 
O seguinte teorema é devido a Jespers e Juriaans.

Teorema 4.31. ([20]) Seja $G$ o produto direto dos grupos $G_{1}$ e $G_{2}, G=G_{1} \times G_{2}$. Então a propriedade do Normalizador vale para $G$ se e somente se ela vale para ambos $G_{1}$ e $G_{2}$.

Demonstração: Seja $\phi_{i}: G \longmapsto G_{i}$ a projeção natural de $G$ em $G_{i}$ e nós também denotaremos por $\phi_{i}$ a sua extensão aos anéis de grupo inteiro. Observe também que se $i \neq j$ então $\operatorname{Ker}\left(\phi_{j}\right)=$ $G_{i}$ ou $\triangle\left(G, G_{i}\right)$ dependendo do significado de $\phi_{i}$.

Suponha que vale $N C$ para $G$ e seja $u \in \mathcal{N}_{\mathcal{U}_{1}\left(\mathbb{Z} G_{i}\right)}\left(G_{i}\right)$. Então naturalmente $u$ pertence ao normalizador de $G$. Portanto, pela hipótese, existem $g \in G$ e $w \in Z_{1}(\mathcal{U}(\mathbb{Z} G))$ tais que $u=g w$. O que por sua vez implica que $u=\phi_{i}(u)=\phi_{i}(w) \phi_{i}(g)$, o que nos mostra que $N C$ vale para $G_{i}$.

Reciprocamente, suponhamos que $N C$ vale para os grupos $G_{1}$ e $G_{2}$ e seja $u \in \mathcal{N}_{\mathcal{U}}(G)$. Denotemos por $u_{i}=\phi_{i}(u)$. Portanto, temos que $u_{i} \in \mathcal{N}_{\mathcal{U}_{1}\left(\mathbb{Z}_{i}\right)}\left(G_{i}\right)$, conseqüentemente existem $g_{i} \in G$ e $w_{i}$ central em $G_{i}$. No entanto, temos que $w_{i}$ é central em $G$, pois $G$ é o produto direto de $G_{1}$ e $G_{2}$. Defina: $w=u u_{1}^{-1} u_{2}^{-1}$. Afirmamos agora que $w$ é um elemento central em $\mathbb{Z} G$. Observe que se $w$ for central em $\mathbb{Z} G$ então $u=u_{2} u_{1} w=g_{2} g_{1} w_{1} w_{2} \in G Z_{1}\left(\mathcal{U}_{1}(\mathbb{Z} G)\right)$.

Afirmamos que $w$ é um elemento central em $\mathbb{Z} G$.

Sejam $g_{i} \in G_{i}$ e $g=w^{-1} g_{i} w$. Então como $w$ normaliza $G$ temos que $g \in G$. Como $g-g_{i}=w^{-1} g_{i} w-g_{i}=\left[w^{-1} g_{i}, w^{-1}\right] \in[\mathbb{Z} G, \mathbb{Z} G]$. Segue pelo lema 1.14 que $g$ e $g_{i}$ são conjugados em $G$. No entanto, como $G_{i}$ é normal em $G$, temos que $g \in G_{i}$. Portanto, $w^{-1} G_{i} w=G_{i}$. Conseqüentemente temos que $w^{-1} g_{i} w=\phi_{i}\left(w^{-1} g_{i} w\right)=g_{i}$, para todo $g_{i}$ em $G_{i}$. Concluindo a demonstração.

Passamos então a demonstração do resultado principal desta subseção.

Demonstração: (do teorema 4.27):

Suponha que $G$ seja um grupo finito, $R(G)$ não trivial. Se $G$ possuir ordem potência de um primo então o resultado segue do teorema 4.28. Então podemos supor $R(G)$ não trivial e que $G$ não possua ordem potência de um primo. Passamos ao estudo dos casos indicados no teorema de Blackburn(teorema 4.26):

(1) Os casos $(b)$ e $(e)$.

Pelos teoremas 4.28 e 4.29 sabemos que $N C$ vale para os $p$-grupos finitos e os grupos de ordem ímpar. Conseqüentemente a tese segue diretamente do teorema 4.31. 
(2) O caso $(d)$ :

O resultado segue diretamente do teorema 4.30.

(3) O caso $(a)$ :

Suponha que $G$ seja um grupo do tipo (a). Nesse caso como $A$ é um grupo abeliano finito ele pode ser representado na forma $A=\left\langle g_{1}\right\rangle \times\left\langle g_{2}\right\rangle \times \cdots \times\left\langle g_{m}\right\rangle$. Seja $v \in \mathcal{N}_{\mathcal{U}}(G)$ então temos que $v^{-1} g_{1} g_{2} \cdots g_{m} v=g_{0}$ para algum $g_{0} \in G$. Além disso, como $g_{0}-g_{1} g_{2} \cdots g_{m}=$ $v^{-1} g_{1} g_{2} \cdots g_{m} v-g_{1} g_{2} \cdots g_{m}=\left[v^{-1} g_{0}, v\right] \in[\mathbb{Z} G, \mathbb{Z} G]$. Segue pelo lema 1.14 que $g_{0}$ é conjugado a $g_{1} g_{2} \cdots g_{m}$ em $G$. Portanto, existe um elemento $a u^{r}$ em $G$ tal que $v^{-1} g_{1} g_{2} \cdots g_{m} v=$ $\left(a u^{r}\right)^{-1} g_{1} g_{2} \cdots g_{m}\left(a u^{r}\right)=u^{-r} g_{1} g_{2} \cdots g_{m} u^{r}$. O que implica que $u^{r} v^{-1} g_{1} g_{2} \cdots g_{m} v u^{-r}=$ $g_{1} g_{2} \cdots g_{m}$. Considere agora $v_{1}=v u^{-r}$, então temos que $v_{1}^{-1} g_{1} v_{1} v_{1}^{-1} g_{2} v_{1} \cdots v_{1}{ }^{-1} g_{m} v_{1}=$ $g_{1} g_{2} \cdots g_{m}$

Afirmamos que $v_{1}^{-1} g_{i} v_{1}=g_{i}$, para todo $i, 1 \leq i \leq m$.

Mostraremos inicialmente que $v_{1}^{-1} g_{i} v_{1} \in\left\langle g_{i}\right\rangle$.

Como $v \in \mathcal{N}_{\mathcal{U}}(G)$. Então temos que $v_{1}^{-1} g_{i} v_{1}=\widetilde{g_{1}}$, para algum $\widetilde{g_{1}} \in G$. Repetindo o raciocínio acima, concluímos que $\widetilde{g_{1}}$ é conjugado a $g_{i}$ em $G$. Portanto, existe um elemento $a^{\prime} u^{r}$ em $G$ tal que $v^{-1} g_{i} v=\left(a^{\prime} u^{r}\right)^{-1} g_{i} a^{\prime} u^{r}=u^{-r} g_{i} u^{r} \in\left\langle g_{i}\right\rangle$, pois sabemos que o subgrupo $\left\langle g_{i}\right\rangle$ é um subgrupo normal de $G$. Conseqüentemente, obtemos que $v_{1}^{-1} g_{i} v_{1}=u^{r} v^{-1} g_{i} v u^{-r} \in\left\langle g_{i}\right\rangle$. Além disso, como $\left\langle g_{i}\right\rangle \cap\left\langle g_{j}\right\rangle=1$ se $i \neq j$ concluímos que $v_{1}{ }^{-1} g_{i} v_{1}=g_{i}$. Segue daí, que $\left[v_{1}, g_{i}\right]=1$, o que por sua vez implica que $\left[v_{1}, a\right]=1$ para todo $a \in A$.

De modo análogo, pelo lema 1.14 temos então que $v_{1}{ }^{-1} u v_{1}=a_{0}{ }^{-1} u^{-r} u u^{r} a_{0}=a_{0}{ }^{-1} u a_{0}$ para algum $a_{0} \in A$.

Considere $v_{2}=v_{1} a_{0}{ }^{-1}$. Afirmamos que $v_{2}$ é uma unidade central. De fato, segue que $\left[v_{2}, u\right]=1$. Além disso, como $\left[v_{2}, a\right]=1$ concluímos que $v_{2}$ é uma unidade central. Conseqüentemente $v=v_{2} a_{0} u^{r} \in G Z_{1}(\mathcal{U})$.

(4) O caso $(c)$ :

Nesse caso, o grupo $G$ é da forma $\langle A, u, t\rangle$, onde $A$ é um grupo abeliano de expoente $k 2^{n}, u^{2} \in A, t^{2}=u^{2^{n}}$ com $o(t)=4$ e $u^{t}=u^{-1}$. Além disso, para todo elemento $x \in A$, $x^{t}=x^{\eta}$ e $x^{u}=x^{\xi}$ onde $\eta=-1+k_{1}\left(2^{n}\right)$ e $\xi=1+k_{2}\left(2^{n}\right)$.

É fácil de observar que se $a \in A$ tal que $o(a) \mid 2^{n}$ então $a^{u}=a$ e $a^{t}=a^{-1}$.

Afirmamos agora que para todo $a \in A$ tal que $o(a) \mid p^{i}$ onde $p$ é um inteiro primo ímpar então $a^{u}=a$ ou $a^{u}=a^{-1}$ e de modo análogo, $a^{t}=a$ ou $a^{t}=a^{-1}$.

Como $u^{2} \in A, A$ abeliano temos que $a=a^{u^{2}}=a^{\left(1+k_{2} 2^{n}\right)^{2}}$ o que implica que:

$$
a^{2^{n} k_{2}\left(2+k_{2} 2^{n}\right)}=1
$$


Observamos que se $k_{2}$ for divisível por $p$ então $2+k_{2} 2^{n}$ não pode ser divisível por $p$. Logo temos que $k_{2}$ é divisível por $p^{i}$ ou $2+k_{2} 2^{n}$ é divisível por $p^{i}$. No primeiro caso, se $k_{2}$ é divisível por $p^{i}$ então $a^{u}=a$ e no caso em $2+k_{2} 2^{n}$ é divisível por $p^{i}$ obtemos que $a^{u}=a^{-1}$.

Com raciocínio análogo, temos que $t^{2} \in A$, donde segue que $a=a^{t^{2}}=a^{\left(-1+k_{1} 2^{n}\right)^{2}}$ o que implica que $a^{2^{n} k_{1}\left(-2+k_{1} 2^{n}\right)}=1$. Conseqüentemente, $k_{1}$ é divisível por $p^{i}$ ou $-2+k_{1} 2^{n}$ é divisível por $p^{i}$. Ou seja, $a^{t}=a^{-1}$ ou $a^{t}=a$.

Para todo $a \in A$ existem $a_{1}, a_{2} \in A$ tais que $a=a_{1} a_{2}, a_{1}^{u}=a_{1}, a_{2}^{u}=a_{2}{ }^{-1}$. De maneira análoga existem $a_{1}^{\prime}, a_{2}^{\prime} \in A$ tais que $a=a_{1}^{\prime} a_{2}^{\prime},\left(a_{1}^{\prime}\right)^{t}=a_{1}^{\prime},\left(a_{2}^{\prime}\right)^{t}=\left(a_{2}^{\prime}\right)^{-1}$.

Considere agora $v \in \mathcal{N}_{\mathcal{U}}(G)$. Então podemos expressar $v$ na forma $v=\alpha_{1}+\alpha_{2} u+\left(\beta_{1}+\beta_{2} u\right) t$, onde $\alpha_{1}, \alpha_{2}, \beta_{1}, \beta_{2} \in \mathbb{Z} A$. Aplicando o epimorfismo canônico $\phi: \mathbb{Z} G \longmapsto \mathbb{Z}(G / A)$ obtemos que $\bar{v}=\epsilon\left(\alpha_{1}\right)+\epsilon\left(\alpha_{2}\right) \bar{u}+\epsilon\left(\beta_{1}\right) \bar{t}+\epsilon\left(\beta_{2}\right) \bar{u} \bar{t}$.

Como o anel de grupo $\mathbb{Z}(G / A)$ possui somente unidades triviais, concluímos que um dos aumentos na expressão de $v$ é igual a \pm 1 e os restantes são iguais a zero. Multiplicando por \pm 1 um elemento do grupo se necessário podemos supor que $\epsilon\left(\alpha_{1}\right)=1$ e $\epsilon\left(\alpha_{2}\right)=\epsilon\left(\beta_{1}\right)=\epsilon\left(\beta_{2}\right)=0$.

Afirmamos inicialmente que $[v, a]=1$ para todo $a \in A$. De fato, como $A \triangleleft G$, temos que $[v, a]=a_{1} \in A$. Portanto, $v a=a v a_{1}$, o que implica que $\alpha_{1} a=a \alpha_{1} a_{1}=\alpha_{1} a a_{1}$. Donde tiramos que $\alpha_{1}\left(1-a_{1}\right)=0$. Conseqüentemente temos que $\epsilon\left(\alpha_{1}\right)$ é divisível pela ordem de $a_{1}$, o que força $a_{1}=1$. Concluímos daí que $[v, a]=1$ para todo $a \in A$.

Consideremos agora $[v, u]$. Como $\langle A, u\rangle \triangleleft G$ temos que $[v, u]=g \in\langle A, u\rangle$. Logo temos que $v u=u v g$ ou $\alpha_{1} u+\alpha_{2} u^{2}+\beta_{1} t u+\beta_{2} u t u=u \alpha_{1} g+u \alpha_{2} u g+u \beta_{1} t g+u \beta_{2} u t g$. Além disso, como $t \notin\langle A, u\rangle$, concluímos que:

$$
\alpha_{1} u+\alpha_{2} u^{2}=u \alpha_{1} g+u \alpha_{2} u g
$$

Suponha agora que $g \notin A$. Então por 4.2 teríamos que $\alpha_{1} u=u \alpha_{2} u g$. Por outro lado sabemos que $\epsilon\left(\alpha_{1} u\right)=1$ e $\epsilon\left(u \alpha_{2} u g\right)=0$. Contradição. Logo temos que $g \in A$ e $[v, u]=g \in A$.

No entanto, pelo lema 1.14 sabemos que $g=[h, u]$ para algum $h \in G$.

Afirmamos a seguir que $g=[a, u]$ para algum $a \in A$.

Por 4.2 temos que:

$$
\alpha_{1} u=u \alpha_{1} g
$$

Seja $\alpha_{1}=\sum \gamma_{a} a$. Por 4.3 temos que $\sum \gamma_{a} a u=u \sum \gamma_{a} a g$. Logo :

$$
\sum \gamma_{a} a g^{-\xi}=\sum \gamma_{a} a^{\xi}
$$

Se $a g^{-\xi}=a_{1}^{\xi}$ então $a_{1}=a^{\xi} g^{-1}$ e por 4.4 teríamos que $\gamma_{a}=\gamma_{a^{\xi} g^{-1}}$ para todo $a \in A$.

Afirmamos que $u g^{-1} u^{-1} g^{-1}=1$. De fato, como $[v, u]=g$ segue que

$$
u g^{-1} u^{-1} g^{-1}=u[v, u]^{-1} u^{-1}[v, u]^{-1}=v^{-1} u v u^{-2} v^{-1} u v .
$$


Como $[v, a]=1$, para todo $a \in A$ e $u^{2} \in A$ temos que $v u^{-2}=u^{-2} v$. Segue por 4.5 que $u g^{-1} u^{-1} g^{-1}=1$. Como $\gamma_{a}=\gamma_{a \xi} g^{-1}$, e $\left(a^{\xi} g^{-1}\right)^{\xi} g^{-1}=a^{\xi^{2}} g^{-\xi} g^{-1}=a u g^{-1} u^{-1} g^{-1}=a$, o que contradiz o fato de $\epsilon\left(\alpha_{1}\right)=1$ a menos que $a=a^{\xi} g^{-1}$ para algum $a \in A$ tal que $\gamma_{a} \neq 0$. Agora mostramos que $g=[a, u]$ para algum $a \in A$. Considere $v_{1}=v a^{-1}$. Como $v$ e $a$ comutam com todo elemento de $A$ o mesmo ocorre com $v_{1}$. Além disso, $\left[v_{1}, u\right]=\left[v a^{-1}, u\right]=a[v, u] u^{-1} a^{-1} u=1$.

Desde que $v$ e $v_{1}$ possuem o mesmo aumento, podemos considerar $v$ como $v_{1}$. Vamos considerar agora $[v, t]$. Seja $g_{0}=[v, t], g_{0} \in G$. Então temos que $v t=t v g_{0}$ o qual nos mostra que $\alpha_{1} t+\alpha_{2} u t+\beta_{1} t^{2}+\beta_{2} u t^{2}=t \alpha_{1} g_{0}+t \alpha_{2} u g_{0}+t \beta_{1} t g_{0}+t \beta_{2} u t g_{0}$. Repetindo o argumento com o aumento que fizemos anteriormente obtemos que

$$
\alpha_{1} t=t \alpha_{1} g_{0}
$$

forçando $g_{0} \in A$. A seguir, afirmamos que $g_{0}$ é um comutador dos elementos do grupo e que $g_{0}=\left[a_{0}, t\right]$ para algum $a \in A$. (A demonstração é análoga a usada anteriormente quando mostramos que $\left(g=[a, u]\right.$ quando $\left.\alpha_{1} u=u \alpha_{1} g\right)$.

Como anteriormente vimos que para todo $a_{0} \in A$, existem $a_{1}^{\prime}, a_{2}^{\prime} \in A$ tais que $a_{0}=a_{1}^{\prime} a_{2}^{\prime},\left(a_{1}^{\prime}\right)^{t}=$ $a_{1}^{\prime},\left(a_{2}^{\prime}\right)^{t}=\left(a_{2}^{\prime}\right)^{-1}$. Podemos assumir que $a_{0}^{t}=a_{0}^{-1}$ e que $a_{0}=a_{1} a_{2}$ tais que $a_{1}^{u}=a_{1}, a_{2}^{u}=a_{2}^{-1}$, onde a ordem de $a_{2}$ não é igual a 2 . Por 4.6 segue que

$$
\alpha_{1} t=t \alpha_{1} g_{0}=t \alpha_{1} a_{0}^{-2}=t \alpha_{1} a_{1}^{-2} a_{2}^{-2} .
$$

Relembrando também que $[v, u]=1$, e portanto, $\alpha_{1} u=u \alpha_{1}$. Por 4.7 temos que $\alpha_{1} t u^{-1}=$ $\alpha_{1} u t=u \alpha_{1} t=u\left(t \alpha_{1} a_{1}{ }^{-2} a_{2}{ }^{-2}\right)=t u^{-1} \alpha_{1} a_{1}{ }^{-2} a_{2}{ }^{-2}=t \alpha_{1} a_{1}{ }^{-2} a_{2}{ }^{2} u^{-1}$. O que implica que

$$
\alpha_{1} t=t \alpha_{1} a_{1}^{-2} a_{2}^{2}
$$

Agora combinando os resultados de 4.7 e 4.8 obtemos que $t \alpha_{1} a_{1}{ }^{-2} a_{2}{ }^{-2}=t \alpha_{1} a_{1}{ }^{-2} a_{2}{ }^{2}$. Portanto, $\alpha_{1}\left(1-a_{2}^{4}\right)=0$. O que significa que $\epsilon\left(\alpha_{1}\right)$ é divisível pela ordem de $a_{2}^{4}$, o que força $a_{2}^{4}=1$. Como todos os 2-elementos de $A$ comutam com $u$, temos que $a_{2}$ não pode ser um 2 -elemento, donde concluímos que $a_{2}=1$. Isso nos diz que $a_{0}^{u}=a_{0}$ ou $\left[a_{0}, u\right]=1$. Consideremos $v_{2}=v a_{0}^{-1}$. Como $v$ e $a_{0}$ comutam com todos os elementos de $A$ e com $u$, segue que o mesmo ocorre com $v_{2}$. Além disso, $\left[v_{2}, t\right]=\left[v a_{0}^{-1}, t\right]=a_{0}[v, t] t^{-1} a_{0}^{-1} t=a_{0}\left[a_{0}, t\right] t^{-1} a_{0}^{-1} t=1$. O que mostra que $v_{2}$ é uma unidade central de $\mathbb{Z} G$. Completando a demonstração.

\subsubsection{O resultado principal}

Finalmente, após utilizarmos os resultados das subseções anteriores procuraremos dar uma caracterização do hipercentro de $\mathcal{U}_{1}(\mathbb{Z} G)$ de um grupo finito $G$. 
Antes de demonstrarmos o resultado principal desta subseção, apresentaremos alguns lemas e teoremas:

Lema 4.32. Se $G$ é um grupo finito e vale $N C$ para $G$ então $Z_{2}(\mathcal{U})=T_{1} Z_{1}(\mathcal{U})$, onde $T_{1}=$ $G \cap Z_{2}(\mathcal{U})$.

Demonstração: Idêntica a demonstração do lema 4.8.

Proposição 4.33. Seja $G$ um grupo finito.Então:

(1) Se $H$ é um subgrupo normal de $G$ então $\left[Z_{2}(\mathcal{U}), H\right] \leq Z_{1}(H)$.

(2) Se $G$ não é um grupo de Dedekind, então: $\left[Z_{2}(\mathcal{U}), G\right] \leq R(G) \cap G^{\prime}$.

Em particular, se $R(G) \cap G^{\prime}=(1)$, então $Z_{1}(\mathcal{U})=Z_{2}(\mathcal{U})$.

Demonstração: (1) Pelo lema 4.17(1) temos que $\left[Z_{2}(\mathcal{U}), H\right] \leq Z_{1}(G)$. Considere o epimorfismo canônico $\varphi: \mathbb{Z} G \longmapsto \mathbb{Z}(G / H)$. Do qual temos que $\varphi\left(\left[Z_{2}(\mathcal{U}), H\right]\right)=\overline{\left[Z_{2}(\mathcal{U}), H\right]}=\overline{1}$ o que implica que $\left[Z_{2}(\mathcal{U}), H\right]-1 \in \operatorname{Ker}(\varphi)$, donde concluímos que $\left[Z_{2}(\mathcal{U}), H\right] \leq Z_{1}(H)$.

(2) Com raciocínio análogo utilizando o epimorfismo canônico $\varphi: \mathbb{Z} G \longmapsto \mathbb{Z}\left(G / G^{\prime}\right)$, obtemos que $\left[Z_{2}(\mathcal{U}), G\right] \leq G^{\prime}$.

Sejam $g, h, k$, elementos de $G$ tais que $h^{-1} g h \notin\langle g\rangle, k^{-1} g k \in\langle g\rangle, o(g)=n$. Então: $1+k \widehat{g} h^{-1}(1-g)$ é um elemento unipotente não-trivial de $\mathcal{U}$. Portanto, pela proposição 4.17(3) segue que para todo $x \in Z_{2}(\mathcal{U})$ temos que:

$$
\left(1+k \widehat{g} h^{-1}(1-g)\right) x=x\left(1+k \widehat{g} h^{-1}(1-g)\right) .
$$

Além disso, como $x^{-1} G x \leq G$ e $h^{-1} g h \notin\langle g\rangle$, temos que:

$$
x^{-1} k \widehat{g} h^{-1} x=k \widehat{g} h^{-1}
$$

Em particular, obtemos que: $x^{-1} \widehat{g} h^{-1} x=\widehat{g} h^{-1}$. O qual implica que $[x, h] \in\langle g\rangle$. Conseqüentemente, $x^{-1} k \widehat{g} x=k \widehat{g}$.

Segue daí que $[x, k] \in\langle g\rangle$ e $\left[Z_{2}(\mathcal{U}), G\right] \leq R(G)$.

Concluímos então que: $\left[Z_{2}(\mathcal{U}), G\right] \leq R(G) \cap G^{\prime}$. 
Finalmente estamos prontos para demonstrar o teorema:

Teorema 4.34. Seja $G$ um grupo finito. Então $Z_{2}(\mathcal{U})=T\left(Z_{2}(\mathcal{U})\right) Z_{1}(\mathcal{U})=T\left(Z_{2}(G)\right) Z_{1}(\mathcal{U})$.

Demonstração: Passemos a analisar os casos possíveis:

Se $G$ for um $p$-grupo então pelo teorema de Coleman (teorema 4.28) vale $N C$ para $G$.

Suponhamos agora que $G$ não seja um $p$-grupo.

Se $G$ fosse um grupo de Dedekind abeliano então vale $N C$ para $G$.

Se $G$ fosse um grupo de Dedekind não abeliano, então utilizando a proposição 1.30 e os teoremas de Coleman (teorema 4.28) e de Marciniak (teorema 4.29) concluímos que vale $N C$ para $G$.

Suponha agora que $G$ não seja um grupo de Dedekind e que não tenha ordem potência de um primo.

Nessas condições, se $G$ for um grupo tal que $R(G)=1$ então pela proposição 4.33 segue a tese.

Finalmente se $G$ for um grupo finito, $R(G) \neq 1$ então o resultado segue pelo resultado da seção anterior (teorema 4.27) que vale $N C$ para $G$.

Da análise dos casos possíveis, a tese segue imediatamente do lema 4.32.

\subsection{Caracterização dos grupos finitos $G$ em função da altura central de $\mathcal{U}_{1}(\mathbb{Z} G)$}

Nesta seção intentamos responder a seguinte questão proposta no início do capítulo: Se a altura central de $\mathcal{U}_{1}(\mathbb{Z} G)$ é no máximo 2. Então quais são os grupos finitos $G$ que possuem altura central exatamente 0,1 e 2 ?

Pelo resultado da seção anterior podemos concluir que $Z_{2}^{2}(\mathcal{U}) \leq Z_{1}(\mathcal{U})$ e que $\left[Z_{2}(\mathcal{U}): Z_{1}(\mathcal{U})\right] \leq 4$. Unindo os resultados dos teorema 3.21 que nos fornece a estrutura do subgrupo de torção $T\left(Z_{2}(\mathcal{U})\right)$ e combinando esse resultado com o teorema 4.24 que nos dá uma descrição dos grupos finitos cujo grupo de unidades do anel de grupo inteiro possuem unidades centrais triviais, obtemos a resposta à pergunta proposta nesta seção através do seguinte:

Corolário 4.35. Sejam $G$ um grupo finito e $\mathcal{U}_{1}(\mathbb{Z} G)$ o grupo de unidades de aumento 1 do anel de grupo inteiro $\mathbb{Z} G$. Então:

(1) $\mathcal{U}_{1}(\mathbb{Z} G)$ possui altura central 2 se e somente se $G$ é um $Q^{*}$-grupo; 
(2) $\mathcal{U}_{1}(\mathbb{Z} G)$ possui altura central 0 se e somente se $G$ possui a propriedade que para todo $g \in G$ e todo número natural $j$, relativamente primo com $|G|, g^{j}$ é conjugado a $g$ ou a $g^{-1} e$ $Z_{1}(G)=(1)$

(3) Se nenhum dos casos acima ocorrer, $\mathcal{U}_{1}(\mathbb{Z} G)$ possui altura central 1.

\subsubsection{Alguns Exemplos}

Apresentaremos nesta subseção alguns exemplos concretos de grupos finitos $G$ satisfazendo as condições do corolário 4.35 citado acima:

Pelo corolario 4.35 concluímos que $\mathcal{U}=\mathcal{U}(\mathbb{Z} G)$ possui altura central 2 se e somente se $G$ é um $Q^{*}$-grupo.

Considere a seguir o grupo $G=S_{n}, n \geq 3$, o grupo simétrico com $n$ elementos. Não é dificil de observar que neste caso, $S_{n}$ satisfaz as condições do teorema de Ritter-Sehgal (teorema 4.24), o que implica que as unidades centrais de $\mathbb{Z} S_{n}$ são triviais. Utilizando o fato bem conhecido que se $n \geq 3, Z\left(S_{n}\right)=1$, podemos concluir pelo corolário 4.35 que nestas condições $S_{n}$ possui altura central 0 .

Pelo corolário 4.12 (do teorema de Gross) segue que se $G$ é um grupo finito, não-solúvel então a altura central de $\mathcal{U}$ é no máximo 1 . Considere a seguir os grupo $A_{5}$ e $A_{6}$, onde $A_{n}$ indica o grupo alternado de $n$ elementos. Em [1], Aleev afirma e demonstra que o centro de $\mathcal{U}\left(\mathbb{Z} A_{5}\right)$ é gerado por um elemento $u$ de ordem infinita, apresentando uma descrição explícita deste gerador (ele demonstra também que o centro de $\mathcal{U}\left(\mathbb{Z} A_{6}\right)$ é um grupo cíclico de ordem infinita, explicitando o gerador $u$ ). Conseqüentemente, podemos concluir pelo corolário 4.12 que a altura central de $\mathcal{U}\left(\mathbb{Z} A_{5}\right)$ (idem para $\left.\mathcal{U}\left(\mathbb{Z} A_{6}\right)\right)$ é 1 .

Consideremos a seguir um grupo $G$ de ordem 27. Como $G$ satisfaz as condições do teorema de Ritter-Sehgal(veja [27] ou [31], p.23) e sendo $G$ um grupo nilpotente finito, concluímos que $Z(G)$ não é trivial, o que implica pelo corolário 4.35 que $\mathcal{U}(\mathbb{Z} G)$ possui altura central 1.

A seguir, temos $G$ definido pelas seguintes relações: $G=\left\langle x, y \mid x^{7}=1=y^{3}, x^{y}=x^{2}\right\rangle$. Como $G$ satisfaz as condições do teorema de Ritter-Sehgal(veja [27] ou [31], p.23) e $Z(G)$ não é trivial, segue pelo corolário 4.35 que $\mathcal{U}(\mathbb{Z} G)$ possui altura central 1 .

Para concluirmos, observamos que se $G$ for um grupo arbitrário então não é necessariamente verdade, que a altura central de $\mathcal{U}$ é no máximo 2. Consideremos o seguinte lema:

Lema 4.36. Seja $G$ grupo nilpotente livre de torção. Então $\mathcal{U}(\mathbb{Z} G)= \pm G$.

Pelo lema 4.36 temos que cada termo $Z_{\alpha}(\mathcal{U})$ da série central superior de $\mathcal{U}$ é igual a $Z_{\alpha}(G)$. A seguir, utilizando o fato de que existem grupos $G$ com classe de nilpotência prescrita concluímos 
que podemos obter infinitos exemplos de grupos $G$ tais que a altura central de $\mathcal{U}$ é maior ou igual a 2. 


\section{Capítulo 5}

\section{A altura central de $\mathcal{U}_{1}(\mathbb{Z} G)$ de um grupo periódico}

Ao introduzir o conceito de $n$-centro de um grupo, Baer percebeu que este conceito compartilhava de uma série de propriedade com o centro de um grupo. Ao estudarem mais profundamente estas semelhanças com o centro, Newell e Kappe obtiveram algumas caracterizações sobre o $n$-centro cujos resultados possuem conexão com uma conjectura de Hall sobre margens.

Neste capítulo introduzindo o conceito de $n$-centro de um grupo, apresentaremos a generalização do resultado do capítulo anterior para o caso de um grupo periódico $G$ de que a altura central de $\mathcal{U}_{1}(\mathbb{Z} G)$ é no máximo 2 e utilizando os resultados sobre o hipercentro de $\mathcal{U}_{1}(\mathbb{Z} G)$ obtemos uma caracterização para o $n$-centro de $\mathcal{U}_{1}(\mathbb{Z} G)$.

É interessante notar aqui a relação como um conceito que teve conexões com uma conjectura sobre margens, é utilizado para obtermos uma caracterização sobre o hipercentro de $\mathcal{U}_{1}(\mathbb{Z} G)$.

Nas condições do Teorema 3.21 segue que:

Corolário 5.1. Seja $G$ um grupo periódico então $T \leq Z_{2}(G)$, onde $T$ denota o subgrupo dos elementos de torção do hipercentro de $\mathcal{U}$.

\subsection{A altura central de $\mathcal{U}_{1}(\mathbb{Z} G)$ é no máximo 2}

Da mesma maneira que no caso finito, nosso objetivo nesta seção é o de mostrarmos que se $G$ for um grupo periódico, então a altura central de $\mathcal{U}_{1}(\mathbb{Z} G)$ é no máximo 2 (teorema 5.10).

Lema 5.2. Seja $G$ um grupo periódico. Então $Z_{2}(\mathcal{U}) \subseteq N_{\mathcal{U}}(G)$.

Demonstração: Sejam $v \in Z_{2}(\mathcal{U})$ e $g \in G$. Então $[v, g]=v^{-1} g^{-1} v g=c$ para algum $c \in Z_{1}(\mathcal{U})$. 
Sendo $G$ um grupo periódico concluímos que existe $m \in \mathbb{N}$ tal que $g^{m}=1$. Portanto, utilizando a proposição 1.33 obtemos que $1=[v, g]^{m}=\left[v, g^{m}\right]=c^{m}$. Concluímos que $c$ é um elemento de ordem finita e pelo lema 1.16, temos que $c$ é uma unidade trivial. Conseqüentemente, $v g v^{-1}=c g$ e isso nos leva ao resultado desejado.

Lema 5.3. Seja $G$ um grupo periódico. Então $\left[Z_{2}(\mathcal{U}), \mathcal{U}\right] \subseteq Z_{1}(G)$.

Demonstração: Sejam $u_{2} \in Z_{2}(\mathcal{U}), u \in \mathcal{U}$. Então pelo lema 1.23 temos que $u_{2}^{2}=a g$ para algum $a \in Z_{1}(\mathcal{U})$ e algum $g \in G$. Conseqüentemente sendo $G$ periódico temos que existe um inteiro $n\left(u_{2}\right)$ tal que $u_{2}^{n\left(u_{2}\right)} \in Z_{1}(\mathcal{U})$. No entanto, temos que $\left[u_{2}, u\right]=c$ para algum $c \in$ $Z_{1}(\mathcal{U})$. Novamente utilizando a proposição 1.33 e elevando ambos os membros da igualdade a $n\left(u_{2}\right)$-ésima potência, obtemos que: $1=\left[u_{2}, u\right]^{n\left(u_{2}\right)}=\left[u_{2}^{n\left(u_{2}\right)}, u\right]=c^{n\left(u_{2}\right)}$. Donde concluímos pelo lema 1.16 que $c$ é uma unidade trivial. Concluindo a demonstração.

Lema 5.4. Seja $G$ um grupo periódico. Então $Z_{n+1}^{2}(\mathcal{U}) \subseteq Z_{n}(\mathcal{U})$, para todo $n \geq 1$.

Demonstração: Mostraremos inicialmente que $Z_{2}^{2}(\mathcal{U}) \subseteq Z_{1}(\mathcal{U})$. Suponha que $Z_{2}^{2}(\mathcal{U}) \nsubseteq Z_{1}(\mathcal{U})$. Pelo lema 5.3 e pelo lema 1.23 concluímos que $Z_{2}^{2}(\mathcal{U}) \subseteq N_{\mathcal{U}}^{2}(G) \subseteq G Z_{1}(\mathcal{U})$. Segue daí que existe um elemento $g \in Z_{2}(\mathcal{U}) \backslash Z_{1}(\mathcal{U})$. Seja $u \in \mathcal{U}$. Então pelo lema $5.3,[u, g]=g_{0} \in Z_{1}(G)$. Pelo lema 1.33, concluímos que existe um inteiro $n=n(u)$ tal que $[u, g]^{n}=\left[u^{n}, g\right]=g_{0}^{n}=1 \mathrm{e}$ conseqüentemente que $u^{n} g u^{-n}=g$. Como $g \in Z_{2}(\mathcal{U}) \backslash Z_{1}(\mathcal{U})$, o grupo $G$ é um $Q^{*}$-grupo e pelo teorema 5.16 segue que o expoente de $Z_{1}(G)$ é igual a 2 . Portanto, para todo $u_{2} \in Z_{2}(\mathcal{U})$ e para todo $g^{\prime} \in G$, nós temos que $\left[u_{2}^{2}, g^{\prime}\right]=\left[u_{2}, g^{\prime}\right]^{2}=g_{0}^{\prime 2}=1$. Concluímos que $u_{2}^{2}$ é uma unidade central e portanto que, $Z_{2}^{2}(\mathcal{U}) \subseteq Z_{1}(\mathcal{U})$. Contradição. Portanto, $Z_{2}^{2}(\mathcal{U}) \subseteq Z_{1}(\mathcal{U})$.

A demonstração continua por indução em $n$. No parágrafo anterior mostramos o caso para $n=1$. Suponha agora que o resultado seja válido para $n=k-1 \geq 1$ e consideremos o caso para $n=k$. Seja $u \in \mathcal{U}$ e $u_{k+1} \in Z_{k+1}(\mathcal{U})$. Então $\left[u_{k+1}, u\right]=u_{k} \in Z_{k}(\mathcal{U})$. Além disso, como $\left[u_{k+1}, u_{k}\right] \in Z_{k-1}(\mathcal{U})$ e utilizando a identidade $\left[u_{k+1}^{2}, u\right]=\left[u_{k+1}, u_{k}\right] u_{k}^{2}$, concluímos pela hipótese de indução que $\left[u_{k+1}^{2}, u\right]=\left[u_{k+1}, u_{k}\right] u_{k}^{2} \in Z_{k-1}(\mathcal{U})$, e portanto concluímos que $u_{k+1}^{2} \in Z_{k}(\mathcal{U})$. Completando a demonstração.

Lema 5.5. Seja $G$ um grupo periódico. Então $\left[Z_{3}(\mathcal{U}), \mathcal{U}\right] \subseteq T$.

Demonstração: Para qualquer $u_{3} \in Z_{3}(\mathcal{U})$ e $u \in \mathcal{U}$ e utilizando os lemas 5.3 e 5.4 concluímos que $\left[u_{3}, u\right]^{2}=\left[u_{3},\left[u_{3}, u\right]\right]^{-1}\left[u_{3}^{2}, u\right] \in Z_{1}(G)$. De fato, pelo lema 5.4 temos que $u_{3}^{2} \in Z_{2}(\mathcal{U})$ e pelo lema 5.3 concluímos que $\left[u_{3},\left[u_{3}, u\right]\right]^{-1} \in Z_{1}(G)$ e $\left[u_{3}^{2}, u\right] \in Z_{1}(G)$. Segue imediatamente que $\left[Z_{3}(\mathcal{U}), \mathcal{U}\right] \subseteq T$. 
Lema 5.6. Seja $G$ um grupo periódico e denotemos por $\mathcal{B}_{1}$ o subgrupo de $\mathcal{U}(\mathbb{Z} G)$ gerado por todas as unidades bicíclicas. Então $\left[Z_{3}(\mathcal{U}), \mathcal{B}_{1}\right]=1$.

Demonstração: Mostraremos inicialmente que $\left[Z_{2}(\mathcal{U}), \mathcal{B}_{1}\right]=1$.

Sejam $u_{2} \in Z_{2}(\mathcal{U})$ e $u_{b, a}=1+(1-b) a \widehat{b}$ uma unidade bicíclica. Pelo lema 5.3 então $\left[u_{2}, u_{b, a}\right]=c_{0} \in Z_{1}(G)$. Logo existe um inteiro positivo $n$ tal que $\left[u_{2}, u_{b, a}\right]^{n}=c_{0}^{n}=1 \mathrm{e}$ pelo lema 1.33 segue que $\left[u_{2}, u_{b, a}^{n}\right]=1$. Observando o fato que $u_{b, a}^{n}=1+n(1-b) a \widehat{b}$ nós concluímos que $\left[u_{2}, u_{b, a}\right]=1$. De fato, $\left[u_{2}, u_{b, a}^{n}\right]=1 \Rightarrow u_{2} u_{b, a}^{n}=u_{b, a}^{n} u_{2} \Rightarrow u_{2}(1+n(1-b) a \widehat{b})=$ $\left.(1+n(1-b) a \widehat{b}) u_{2} \Rightarrow n\left(u_{2}(1-b) a \widehat{b}-(1-b) a \widehat{b} u_{2}\right)=0 \Rightarrow u_{2}(1-b) a \widehat{b}=(1-b) a \widehat{b} u_{2}\right) \Rightarrow$ $\left.u_{2}+u_{2}(1-b) a \widehat{b}=u_{2}+(1-b) a \widehat{b} u_{2}\right) \Rightarrow\left[u_{2}, u_{b, a}\right]=1$.

A seguir sejam $u_{3} \in Z_{3}(\mathcal{U})$ e $b$ uma unidade bicíclica. Então pelo lema 5.5, $\left[u_{3}, b\right] \in T$ e portanto, $\left[u_{3}, b\right]^{n}=1$ para algum inteiro positivo $n$. Observando que $\left[u_{3}, b^{n}\right]=\left[u_{3}, b^{n-1}\right] b^{n-1}\left[u_{3}, b\right] b^{-(n-1)}=$ $\left[u_{3}, b^{n-1}\right]\left[b^{n-1},\left[u_{3}, b\right]\right]\left[u_{3}, b\right]$ e utilizando o fato que $\left[b^{n-1},\left[u_{3}, b\right]\right]=1$, pois $\left[u_{3}, b\right] \in Z_{2}(\mathcal{U})$, nós concluímos por indução, que $\left[u_{3}, b^{n}\right]=\left[u_{3}, b^{n-1}\right]\left[u_{3}, b\right]=\left[u_{3}, b\right]^{n-1}\left[u_{3}, b\right]=\left[u_{3}, b\right]^{n}=1$. Portanto, $\left[u_{3}, b\right]=1$ como vimos anteriormente.

Relembramos a definição de $Q^{*}$-grupo.

Definição 5.7. Diremos que $G$ é um $Q^{*}$-grupo se $G$ possuir um subgrupo normal abeliano $L$ de índice 2 com um elemento a de ordem 4 tal que para todo $g \notin L, g^{2}=\boldsymbol{a}^{2}$ e $g^{-1} h g=h^{-1}$, para todo $h$ em $L$.

Lema 5.8. Seja $G$ um $Q^{*}$-grupo. Então: $\left[Z_{3}(\mathcal{U}), G\right] \subseteq\left\langle a^{2}\right\rangle$.

Demonstração: A demonstração é idêntica ao lema 4.20, com a única diferença que aqui empregamos o lema 5.6 no lugar da proposição 4.17(3).

Uma conseqüência imediata do lema é o:

Corolário 5.9. Seja $G$ um $Q^{*}$-grupo então $\left[Z_{3}^{2}(\mathcal{U}), G\right]=1$ e $Z_{3}^{2}(\mathcal{U}) \subseteq Z_{1}(\mathcal{U})$.

Estamos prontos para demonstrar o teorema 5.10.

Teorema 5.10. Seja $G$ um grupo periódico. Então: $Z_{3}(\mathcal{U})=Z_{2}(\mathcal{U})$.

Demonstração: De acordo com o teorema 3.21 temos de considerar três casos: 
(1) Suponhamos que $G$ seja um 2-grupo Hamiltoniano. Então pela proposição 1.30 e pelo lema $1.19, \mathcal{U}= \pm G= \pm T=Z_{2}(\mathcal{U})$.

(2) Suponha que $T$ é um subgrupo central de $\mathcal{U}$. Então o resultado decorre diretamente do lema 5.5 .

(3) Suponhamos agora que $T$ é abeliano, mas não seja um subgrupo central. Nesse caso, $G=\langle H, b\rangle$ é um grupo do tipo (3) no teorema 3.21, ou seja, $G$ é um $Q^{*}$-grupo, portanto, $T=\langle a\rangle \oplus E=Z_{2}(\mathcal{U}) \cap Z_{2}(G)$. Neste caso, relembramos que $Z_{1}(G)=\left\{x \in G \mid x^{2}=1\right\}$ e o expoente de $T$ é igual a 4 .

Suponha por absurdo que exista $x \in Z_{3}(\mathcal{U}) \backslash Z_{2}(\mathcal{U})$. Então para algum $u \in \mathcal{U},[x, u]=t \in T$ é um elemento de ordem 4. Claramente $t \in G^{\prime}$ o grupo derivado de $G$. Além disso, não é difícil de verificarmos neste caso que $G^{\prime}=\left\{h^{2} \mid h \in H\right\}$. Para observarmos o primeiro fato, considere a projeção natural $\varphi: \mathbb{Z} G \longmapsto \mathbb{Z}\left(G / G^{\prime}\right)$, aplicando $\varphi$ a $t$ obtemos que $\varphi(t)=\bar{t}$, decorre daí que: $[\bar{x}, \bar{u}]=\bar{t}=\overline{1}$, pois $\mathbb{Z}\left(G / G^{\prime}\right)$ é um anel de grupo comutativo. Portanto, $t-1 \in \triangle\left(G^{\prime}\right) \mathbb{Z} G$ o que implica que $t \in G^{\prime}$.

Já vimos anteriormente que $\langle a\rangle \triangleleft \mathcal{U}$, (teorema 3.20), o(a)=4, então $a^{2} \in Z(\mathcal{U})$.

Mostraremos agora que $G^{\prime}=\left\{h^{2} \mid h \in H\right\}$. Para isto mostraremos que para quaisquer $g_{1} \in G, g_{2} \in G$, existe $h \in H$ tal que $\left[g_{1}, g_{2}\right]=h^{2}$.

(a) Se $g_{1} \in H, g_{2} \in H$ então nada há a demonstrar.

(b) Suponha agora que $g_{1} \in G \in H, g_{2} \in G \backslash H$.

Como $H$ é um subgrupo de $G$ de índice 2, todo $g \in G \backslash H$ pode ser expresso na forma $g=h b$, para algum $h \in H$.

Nesse caso, pela proposição 1.32(ii), temos que:

$\left[g_{1}, g_{2}\right]=\left[h_{1}, h_{2} b\right]=\left[h_{1}, h_{2}\right]^{b}\left[h_{1}, b\right]=h_{1}^{-1} h_{1}^{-1}=h_{1}^{-2}$.

(c) Se $g_{1} \in G \backslash H, g_{2} \in G \backslash H$ então:

$$
\begin{aligned}
{\left[g_{1}, g_{2}\right] } & =\left[h_{1} b, h_{2} b\right] \\
& =\left[h_{1}, h_{2} b\right]^{b}\left[b, h_{2} b\right] \\
& =\left[\left[h_{1}, h_{2} b\right]^{b}\left[h_{1} b\right]\right]^{b}\left[b, h_{2}\right]^{b} \\
& =\left[h_{1} b\right]^{b}\left[b, h_{2}\right]^{b} \\
& =\left[h_{1}^{-2}\right]^{b}\left[h_{2}^{-2}\right]^{b} \\
& =\left(h_{2}^{-2}\right)\left(h_{1}^{2}\right) \\
& =\left(h_{2}^{-1} h_{1}\right)^{2} .
\end{aligned}
$$


Portanto, podemos observar que para quaisquer $g_{1} \in G, g_{2} \in G$, existe $h \in H$ tal que $\left[g_{1}, g_{2}\right]=h^{2}$.

Logo, $G^{\prime}=\left\{h^{2} \mid h \in H\right\}$.

Perceba que pelo lema $5.8[x, h] \in\left\langle a^{2}\right\rangle \subseteq Z_{1}(\mathcal{U})$, para todo $h \in H$. Logo, $[x, h]^{2}=1$. Segue que $[x, t]=\left[x, h^{2}\right]$ (para algum $\left.h \in H\right)=[x, h] h[x, h] h^{-1}=[x, h]^{2}=1$.

Portanto, $\left[x^{2}, u\right]=x[x, u] x^{-1}[x, u]=[x, t] t^{2}=t^{2} \neq 1$. No entanto, pelo corolário 5.9, nós temos que $\left[x^{2}, u\right]=1$. Contradição. Donde concluímos que: $Z_{3}(\mathcal{U})=Z_{2}(\mathcal{U})$ sempre.

Corolário 5.11. Seja $G$ um grupo periódico. Se todas as unidades centrais são triviais, então todas as unidades hipercentrais são triviais também.

Demonstração: Seja $u \in \widetilde{Z}(\mathcal{U})$. Então pelo teorema 5.10 e pelo lema 5.2 temos que $u \in \mathcal{N}_{\mathcal{U}}(G)$. Pelo lema 1.20 segue que $u u^{*}=g \in Z_{1}(G)$ o que implica que $u u^{*}=1$. Donde $u$ é uma unidade trivial pelo lema 1.17, finalizando a demonstração.

\subsection{O $n$-centro do grupo de unidades de um anel de grupo inteiro}

Introduzimos o conceito de $n$-centro de um grupo e nosso objetivo principal é o de obtermos uma caracterização do $n$-centro de $\mathcal{U}_{1}(\mathbb{Z} G)$ em função dos resultados que obtivemos acerca do hipercentro de $\mathcal{U}_{1}(\mathbb{Z} G)$. Em particular, apresentamos uma caracterização dos $Q^{*}$-grupos, como aqueles grupos periódicos que possuem um elemento não-central que pertence ao 4 -centro de $\mathcal{U}$. Além de estudarmos algumas relações entre o grupo das unidades de torção do $n$-centro de $\mathcal{U}$ e o grupo das unidades de torção do hipercentro de $\mathcal{U}$.

\subsubsection{Alguns resultados básicos e notações}

Sejam

$$
S_{1}(G, n)=\left\{a \in G \mid(a x)^{n}=a^{n} x^{n}, \forall x \in G\right\}
$$

$\mathrm{e}$

$$
S_{2}(G, n)=\left\{a \in G \mid(x a)^{n}=x^{n} a^{n}, \forall x \in G\right\} .
$$


Em [4] Baer definiu o $n$-centro como

$$
Z(G, n)=S_{1}(G, n) \cap S_{2}(G, n) .
$$

No entanto, veremos que apenas uma das condições de $n$-comutatividade é suficiente para definirmos o $n$-centro devido ao seguinte teorema.

Teorema 5.12. Para um inteiro $n$ e um grupo $G$ qualquer

$$
Z(G, n)=S_{1}(G, n)=S_{2}(G, n)
$$

Demonstração: Afirmamos inicialmente que $S_{1}(G, n) \subseteq S_{2}(G, n)$.

Seja $a \in S_{1}(G, n)$ então tomando inversos obtemos que $\left(x^{-1} a^{-1}\right)^{n}=x^{-n} a^{-n}$, para todo $x$ em $G$ e $\left(y a^{-1}\right)^{n}=y^{n} a^{-n}$, para todo $y$ em $G$.

Segue daí que:

$\left(a x^{n} a^{-1}=\left(a x a^{-1}\right)^{n}=a^{n}\left(x a^{-1}\right)^{n}=a^{n} x^{n} a^{-n}\right) \Rightarrow\left(a x^{n} a^{-1}=a^{n} x^{n} a^{-n}\right) \Rightarrow\left(x^{n} a^{-1}=\right.$ $\left.a^{n-1} x^{n} a^{-n}\right) \Rightarrow\left(x^{n}=a^{n-1} x^{n} a^{-(n-1)}\right) \Rightarrow\left(1=a^{n-1} x^{n} a^{-(n-1)} x^{-n}\right)$.

Donde obtemos que $\left[a^{n-1}, x^{n}\right]=1$, para todo $x$ em $G$.

Por outro lado temos que: $a^{n} x^{n}=(a x)^{n}=a(x a)^{n-1} x$. Portanto, $(x a)^{n-1}=a^{n-1} x^{n-1}$. Segue daí que: $(x a)^{n}=(x a)^{n-1}(x a)=a^{n-1} x^{n-1} x a=a^{n-1} x^{n} a=x^{n} a^{n-1} a=x^{n} a^{n}$.

Mostramos que $S_{1}(G, n) \subseteq S_{2}(G, n)$. A outra inclusão é totalmente análoga.

Note que $Z(G, 1)=Z(G, 0)=G$.

Proposição 5.13. Seja $a \in Z(G, n)$. Então:

(1) $\left[a^{n-1}, x^{n}\right]=1$ para todo $x$ em $G$;

(2) $a \in Z(G, 1-n)$ (Portanto, $Z(G, n)=Z(G, 1-n)$ sempre);

(3) $\left[a^{n}, x\right]=[a, x]^{n}=\left[a, x^{n}\right]$ para todo $x$ em $G$;

(4) $1=\left[a, x^{n(1-n)}\right]=\left[a^{n(1-n)}, x\right]=\left[a^{n}, x^{1-n}\right]$ para todo $x$ em $G$;

(5) $a^{n} \in Z(G, n-1)$.

Demonstração: (1) Decorre da demonstração do teorema 5.12; pois $S_{1}(G, n)=Z(G, n)$.

(2) Da demonstração do teorema 5.12 temos que $(x a)^{n-1}=a^{n-1} x^{n-1}$ para $a \in S_{1}(G, n)$. Tomando inversos, $(x a)^{1-n}=x^{1-n} a^{1-n}$ e o resultado segue do teorema 5.12. 
(3) Observando que $[a, x]^{n}=\left(a^{-1} a^{x}\right)^{n}=a^{-n} a^{n x}=\left[a^{n}, x\right]$ a primeira igualdade está demonstrada. Para mostrarmos a segunda igualdade, observamos que $a^{n} x^{-n}$ comuta com $a x^{-1}$ pois $a^{n} x^{-n}=\left(a x^{-1}\right)^{n}$. Portanto, $a^{n+1}\left[a, x^{n}\right] x^{-(n+1)}=a^{n} x^{-n} a x^{-1}=a x^{-1} a^{n} x^{-n}=$ $a^{n+1}\left[a^{n}, x\right] x^{-(n+1)}$ e a segunda igualdade segue.

(4) Por (1), (2) e (3) obtemos que

$1=\left[a^{1-n}, x^{n}\right]=\left[a^{1-n}, x\right]^{n}=[a, x]^{n(1-n)}=\left[a^{n}(1-n), x\right]$; e de maneira análoga, obtemos que: $1=\left[a^{1-n}, x^{n}\right]=\left[a, x^{n(1-n)}\right]=\left[a^{n}, x^{1-n}\right]$.

(5) Por (4) nós observamos que $a^{n}$ e $x^{n-1}$ comutam. Portanto, $\left(a^{n} x\right)^{n-1}=\left(a^{n} x\right)^{n} x^{-1} a^{-n}=a^{n^{2}} x^{n-1} a^{-n}=a^{n(n-1)} x^{n-1}$.

Podemos facilmente ver que o 2 -centro de um grupo coincide com o seu centro.

Proposição 5.14. Seja G um grupo. Então:

$$
Z(G, 3)=\left\{a \in R_{2}(G) \mid a^{3} \in Z_{1}(G)\right\} \quad \text { e } Z(G, 3) \subseteq Z_{3}(G) .
$$

Aqui $Z_{m}(G)$ é o $m$-ésimo centro de $G$ e $R_{m}(G)=\left\{a \in G \mid\left[a,_{m} x\right]=1, \forall x \in G\right\}$ denota o conjunto dos $m$-Engel elementos à direita, onde

$$
\left[x,_{m} y\right]=\left[\left[x,_{m-1} y\right], y\right] \quad e \quad[x, 1 y]=[x, y]
$$

Demonstração: Seja $a \in Z(G, 3)$, então temos que:

$$
\left(x a^{-1}\right)^{3}=x^{1+a+a^{2}} a^{-3}=x^{-3} a^{-3},
$$

onde $x^{g}=g^{-1} x g$. De fato, a primeira igualdade resulta do fato que $\left(x a^{-1}\right)^{3}=x x^{a} x^{a^{2}} a^{-3}$ e a segunda igualdade decorre da definição de 3 -centro de um grupo, comparando o primeiro e o último fator da igualdade.

Segue-se do fato acima que: $x^{a+a^{2}}=x^{2}$. Substituindo $x$ por $x^{-1}$ na igualdade anterior obtemos que $\left(x^{-1}\right)^{a}\left(x^{-1}\right)^{-2}=x^{-2}$ e invertendo obtemos que: $x^{a^{2}} x^{a}=x^{a^{2}+a}=x^{2}$. Portanto,

$$
x^{a+a^{2}}=x^{a^{2}+a} .
$$

Conjugando com $a^{-1}$ decorre que: $\left[x^{a}, x\right]=1$. De fato, temos que: $a\left(x^{a^{2}} x^{a}\right) a^{-1}=a\left(x^{a} x^{a^{2}}\right) a^{-1}$ $\Rightarrow x^{a} x=x x^{a} \Rightarrow\left[x^{a}, x\right]=1$. E portanto, como $\left[x^{a}, x\right]=1$, concluímos que $a \in R_{2}(G)$. A última afirmação provém do fato que: $\left[x^{a}, x\right]=\left(x^{-1}\right)^{a} x^{-1} x^{a} x=[a, x] x^{-1}[a, x]^{-1} x=[[a, x], x]=1$.

Pela proposição 5.13(5) sabemos que $a^{3} \in Z(G, 2)=Z(G)$. Portanto, $Z(G, 3) \subseteq Y$, onde $Y=\left\{a \in R_{2}(G) \mid a^{3} \in Z(G)\right\}$. 
Mostraremos agora que: $Y \subseteq Z(G, 3)$.

Seja $a \in Y$. Então:

$$
[a, x, x]=1=[a, x, a]
$$

$\mathrm{e}$

$$
\left[a^{3}, x\right]=[a, x]^{3} .
$$

Portanto,

$$
\left(a x^{-1}\right)^{3}=a^{3}[a, x]^{3} x^{-3}=a^{3} x^{-3}, \forall x \in G,
$$

logo concluímos que $Y \subseteq Z(G, 3)$. Para justificarmos a primeira igualdade na equação 5.4, observe que pela equação 5.2 concluímos que: $[a, x, x]=[[a, x], x]=1=[a, x, a]=[[a, x], a]$, ou seja $[a, x]$ comuta com $x$ e com $a$, além disso, segue que $\left[[a, x], x^{-1}\right]=1$ pois $\left[[a, x], x^{-1}\right]=$ $[x, a] x[a, x] x^{-1}=[x, a][a, x] x x^{-1}=[a, x]^{-1}[a, x]=1$, pois $[a, x]$ comuta com $x$. Logo,

$$
\left(a x^{-1}\right)^{3}=a^{2}[a, x] x^{-2} a x^{-1}=a^{2}[a, x] a[a, x] x^{-1}[a, x] x^{-2}=a^{3}[a, x]^{3} x^{-3} .
$$

Agora $a^{3} \in Z(G)$ implica que $[a, x, y, z]^{3}=1$, pois $1=\left[a^{3}, x, y, z\right]=\left[\left[a^{3}, x, y\right], z\right]=$ $\left[[\underbrace{\left.a^{3}, x\right]}_{1}, y], z\right]=[\underbrace{\left.[a, x]^{3}, y\right]}_{1}, z]=[\underbrace{[a, x, y]^{3}}_{1}, z]=[a, x, y, z]^{3}=1$.

Segue de [22] que $[a, x, y, z]^{4}=1$. Portanto, $[a, x, y, z]=1, \forall x, y, z \in G$, e $Z(G, 3) \subseteq Z_{3}(G)$.

Teorema 5.15. Seja $G$ um grupo periódico. Então:

$$
Z(\mathcal{U}, 3)=Z(\mathcal{U}, 2)=Z_{1}(\mathcal{U})
$$

Demonstração: Pela proposição 5.14 temos que $Z(\mathcal{U}, 3) \subseteq Z_{3}(\mathcal{U})$, pelo teorema $5.10, Z_{3}(\mathcal{U})=$ $Z_{2}(\mathcal{U})$ e do lema 5.4 temos que $Z_{2}^{2}(\mathcal{U}) \subseteq Z_{3}(\mathcal{U})$. Segue daí que, para todo $u \in Z(\mathcal{U}, 3), u^{2} \in$ $Z_{1}(\mathcal{U})$. Pela proposição 5.14 concluímos que $u^{3} \in Z_{1}(\mathcal{U})$. Portanto, $u \in Z_{1}(\mathcal{U})$ e $Z(\mathcal{U}, 3) \subseteq$ $Z_{1}(\mathcal{U})$.

\subsubsection{Uma caracterização do $n$-centro de $\mathcal{U}_{1}(\mathbb{Z} G)$}

Empregando a notação definida na seção anterior, pretendemos nos concentrar em atingir alguns objetivos principais: Caracterizar o $n$-centro de $\mathcal{U}_{1}(\mathbb{Z} G)$ para $n \geq 4$; caracterizar os $Q^{*}$-grupos como aqueles grupos periódicos que contém um elemento não-central que pertence ao 4 -centro de $\mathcal{U}$.

Com esse intuito, inicialmente apresentamos um teorema de caracterização dos $Q^{*}$-grupos. Logo a seguir, estudamos algumas relações entre o hipercentro de $\mathcal{U}$ de um grupo periódico e 
o $n$-centro de $\mathcal{U}$; e entre o $n$-centro de $\mathcal{U}$ e os $Q^{*}$-grupos. Fatos estes que nos permitirão atingirmos os propósitos estabelecidos nesta subseção.

Teorema 5.16. Seja $G$ um grupo periódico. Então as seguintes afirmações são equivalentes:

(1) G contém um elemento não-central a com a propriedade que dada qualquer unidade $u \in \mathcal{U}$, existe um inteiro positivo $n=n(u)$ tal que $u^{n} a u^{-n}$ pertence a $G$.

(2) Gé um $Q^{*}-$ grupo.

Demonstração: $(1) \Rightarrow(2)$ :

Afirmamos inicialmente que $\langle a\rangle \triangleleft G$.

Seja $y \in G$ e consideremos a unidade bicíclica $u=1+(1-a) y \widehat{a}$.

Calculando $u^{n} a u^{-n}, n \geq 1$, obtemos que:

$u^{n} a u^{-n}=(1+n(1-a) y \widehat{a}) a(1-n(1-a) y \widehat{a})=(1+n(1-a) y \widehat{a})(a-a n(1-a) y \widehat{a})=a-a n(1-$ a) $y \widehat{a}+n(1-a) y \widehat{a}-\underbrace{n(1-a) y \widehat{a} a n(1-a) y \widehat{a}}_{=0, \text { pois }(1-a) \widehat{a}=0}=a-a n(1-a) y \widehat{a}+n(1-a) y=a+n\left(-a+a^{2}+1-a\right) y \widehat{a}=$ $a+n\left(1-2 a+a^{2}\right) y \widehat{a}$.

Por hipótese temos que $u^{n} a u^{-n} \in G$, portanto, $u^{n} a u^{-n}$ é um elemento de ordem finita, o que implica que $\left(1-2 a+a^{2}\right) y \widehat{a}=0$, portanto, $y=a y a^{i}$ para algum $i$. O que nos mostra que $y^{-1} a y \in\langle a\rangle$.

Afirmamos agora que se $y \in G$ então $a$ normaliza $\langle y\rangle$.

Consideremos então a unidade bicíclica $v=1+(1-y) a \widehat{y}$. Se $n \geq 1$ então efetuando cálculos similares aos efetuados anteriormente, obtemos que $v^{n} a v^{-n}=a+n(1-y) a \widehat{y} a-n a(1-y) a \widehat{y}-$ $n^{2}(1-y) a \widehat{y} a(1-y) a \widehat{y}$. Novamente, empregando a hipótese, decorre que: $(1-y) a \widehat{y} a=a(1-y) a \widehat{y}$. Portanto, ou aya $=a^{2} y^{i}$ para algum $i$ ou $a y a=y a y^{j} a$ para algum $j$. No primeiro caso, obtemos que $a^{-1} y a=y^{i}$ e no segundo caso, obtemos que $a^{-1} y a=y^{1-j}$. Em qualquer um dos dois casos, concluímos que $a$ normaliza $G$.

Mostraremos a seguir que se $z \in\langle a, y\rangle$ então $\langle z\rangle \triangleleft\langle a, y\rangle$ e conseqüentemente concluiremos que o grupo $\langle a, y\rangle$ é um grupo de Dedekind.

É suficiente provarmos que $y$ normaliza $\langle z\rangle$, pois já vimos anteriormente que $a$ normaliza $\langle z\rangle$.

Consideremos, agora, a unidade bicíclica $f=1+(1-z) y \widehat{z}$. Como anteriormente nós obtemos que $f^{n} a f^{-n}=a+n(1-z) y \widehat{z} a-n a(1-z) y \widehat{z}-n^{2}(1-z) y \widehat{z} a(1-z) y \widehat{z}$. Novamente a hipótese nos $\operatorname{diz}$ que $(1-z) y \widehat{z} a=a(1-z) y \widehat{z}$. Portanto, $a y=y z^{i} a$ para algum $i$ ou $a y=a z y z^{j}$ para algum $j$. No segundo caso, obtemos que $y=z y z^{j}, \operatorname{logo} y^{-1} z y=z^{-j}$ e $y^{-1} z y \in\langle z\rangle$. No primeiro caso, 
obtemos que: $a^{-1} y^{-1} a y=a^{-1} z^{i} a \in\langle z\rangle$, pois como vimos anteriormente, $a$ normaliza $\langle z\rangle$. Mas também sabemos que $a^{-1} y^{-1} a y \in\langle a\rangle$, pois $\langle a\rangle \triangleleft G$. Segue então que $\left\langle a^{-1} y^{-1} a y\right\rangle \triangleleft G$.

Utilizando a proposição 1.32(ii) concluímos que para quaisquer elementos $r, s \in\langle a, y\rangle$ o elemento $r^{-1} s^{-1} r s \in\left\langle a^{-1} y^{-1} a y\right\rangle$. Conseqüentemente $\langle a, y\rangle^{\prime}=\left\langle a^{-1} y^{-1} a y\right\rangle \subseteq\langle z\rangle$, donde podemos concluir que $\langle z\rangle \triangleleft\langle a, y\rangle$.

Afirmamos, agora, que $y \in G$ então $\langle a, y\rangle$ é abeliano ou $\langle a, y\rangle \cong Q$, onde $Q$ aqui representa o grupo dos quatérnions.

Considerando os resultados anteriores e pela proposição 1.30, a única possibilidade distinta que poderia ocorrer seria que $\langle a, y\rangle \cong Q \times A$, onde $A$ é um grupo abeliano de ordem ímpar.

Suponha que $G=\langle a, y\rangle \cong Q \times A$, onde $Q$ é um grupo quatérnion de ordem 8 e $A$ é um grupo abeliano de ordem ímpar.

Nesse caso podemos expressar $a$ na forma $a=t v, t \in Q, v \in A, o(v)=m$, ímpar. Observamos que $A \subseteq Z(G)$. Como $a \notin Z(G), t$ é um elemento de ordem 4, caso contrário, $o(t)=2$ $\Rightarrow t \in Z(Q) \subseteq Z(Q \times A)$. Contradição. Portanto, $o(a)=o(t) o(v)=4 m$, pois os elementos $t$ e $v$ são co-primos.

Sendo $t$ um elemento de ordem 4 em $Q, t$ não é um elemento central. Logo existe $u \in Q$ tal que $[u, t] \neq 1, \log o o(u)=4$.

Tome um elemento $g_{0}$ distinto de $1, g_{0} \in A \subseteq Z(G), o\left(g_{0}\right)=p, p$ primo ímpar, o qual sempre existe pelo teorema de Cauchy.

Defina $x:=u g_{0}, o(x)=o(u) o\left(g_{0}\right)=4 p$. Além disso, sabemos que $[x, a]=[z, u] \neq 1$. Donde,

$$
a^{-1} x a=(t v)^{-1} u g_{0} t v=t^{-1} u t g_{0}=u^{-1} g_{0}=u^{3} g_{0} .
$$

Por outro lado: $x^{2 p+1}=\left(u g_{0}\right)^{2 p+1}=(u)^{2 p+1}\left(g_{0}\right)^{2 p+1}=(u)^{2 p+1}\left(g_{0}\right)^{2 p}\left(g_{0}\right)=u u^{2 p} g_{0}=$ $u\left(u^{2}\right)^{p} g_{0}={ }^{* *} u u^{2} g_{0}=u^{3} g_{0}$.

$(* *)$ Se $u \in Q, o(u)=4$ então $\left(u^{2}\right)^{p}=1$ ou $\left(u^{2}\right)^{p}=u^{2}$. Se $\left(u^{2}\right)^{p}=1$ então $p$ dividiria 2 . Contradição, pois $p$ é ímpar. Logo $\left(u^{2}\right)^{p}=u^{2}$. Donde, concluimos que $\left.x^{2 p+1}=u^{3} g_{0}{ }^{* * *}\right)$.

Comparando (***) e 5.5 decorre que:

$$
a^{-1} x a=x^{2 p+1} .
$$

Seja $\phi$ a função de Euler, $o(x)=4 p, \phi(4 p)=2(p-1)$, considere a unidade cíclica de Bass não-trivial:

$$
\omega=\left(1+x+x^{2}+\cdots+x^{2 p-2}\right)^{2(p-1)}+\frac{1-(2 p-1)^{2(p-1)}}{4 p} \widehat{x} .
$$


Afirmamos que: $a^{-1} \omega a \neq \omega$.

Suponha que $a^{-1} \omega a=\omega$, e utilizando 5.6, concluímos que:

$\left(1+x+x^{2}+\cdots+x^{2 p-2}\right)^{2(p-1)}=\left(1+x^{2 p+1}+x^{2(2 p+1)}+\cdots+x^{(2 p-2)(2 p+1)}\right)^{2(p-1)}$.

Multiplicando ambos os lados da igualdade por: $(1-x)^{2(p-1)}\left(1-x^{-1}\right)^{2(p-1)}$, nós obtemos:

$$
\begin{aligned}
\left(1-x^{2(p-1)}\right)^{2(p-1)}\left(1-x^{2(p+1)}\right)^{2(p-1)} & =(1-x)^{2(p-1)}\left(1-x^{-1}\right)^{2(p-1)} \\
\left(2-x^{2 p-1}-x^{2 p+1}\right)^{2(p-1)} & =\left(2-x-x^{-1}\right)^{2(p-1)} \\
\left(2 x^{2 p}-x^{-1}-x\right)^{2(p-1)} & =\left(2-x-x^{-1}\right)^{2(p-1)} .
\end{aligned}
$$

No entanto, comparando os coeficientes de $x^{2 p-1}$ em ambos os lados da igualdade acima, obtemos que o coeficiente de $x^{2 p-1}$ no lado esquerdo da igualdade é não-nulo e negativo enquanto que o coeficiente de $x^{2 p-1}$ no lado direito é nulo. Contradição. Logo, $a^{-1} \omega a \neq \omega$.

A seguir observamos que

$$
\omega=x^{2}+\left(1-x^{2 p}\right) t, \text { para algum } \mathrm{t} \in \mathbb{Z}\langle x\rangle .
$$

De fato, considere a projeção natural $\phi: \mathbb{Z}(\langle x\rangle) \longmapsto \mathbb{Z}\left(\langle x\rangle /\left\langle x^{2 p}\right\rangle\right)$. Aplicando $\phi$ a $\omega$ obtemos que $\phi(\omega)=\bar{\omega}=\left(\widehat{\bar{x}}-\bar{x}^{2 p-1}\right)^{2(p-1)}+\frac{1-(2 p-1)^{2(p-1)}}{2 p} \widehat{\bar{x}}$.

E calculando diretamente obtemos que $\bar{\omega}=\bar{x}^{2}$.

Afirmamos que os conjugados da forma $\omega^{n} a \omega^{-n}$ não pertencem a $G$.

Consideremos inicialmente $\omega a \omega^{-1}$ e expressamos $\omega$ como $\omega=\omega_{1}+\omega_{2}$, onde $\omega_{1}$ é a soma dos termos de $\omega$ envolvendo potências ímpares de $x$ e $\omega_{2}$ é a soma dos termos de $\omega$ envolvendo potências pares de $x$. De maneira análoga, expressamos $\omega^{-1}$ como $\omega^{-1}=\omega_{1}^{1}+\omega_{2}^{1}$.

Observando agora a caracterização dada em 5.8, concluímos diretamente que $\omega_{2} a=a \omega_{2}$ e que $\omega_{1} a=-a \omega_{1}$. Relações que nos permitem concluir que:

$$
\begin{aligned}
\omega a \omega^{-1} & =\left(\omega_{1}+\omega_{2}\right) a\left(\omega_{1}^{1}+\omega_{2}^{1}\right) \\
& =a\left(\omega_{2}-\omega_{1}\right)\left(\omega_{1}^{1}+\omega_{2}^{1}\right) \\
& =a\left(1+2\left(\omega_{2} \omega_{1}^{1}-\omega_{1} \omega_{1}^{1}\right)\right) .
\end{aligned}
$$

Anteriormente observamos que $\omega a \omega^{-1} \neq a$, o que implica que a unidade $m=1+2\left(\omega_{2} \omega_{1}^{1}-\right.$ $\left.\omega_{1} \omega_{1}^{1}\right)$ não está em $\pm\langle x\rangle$ e portanto, pelo lema 1.17, $m$ é uma unidade de ordem infinita. Observando agora que para todo $n \geq 1, \omega^{n} a \omega^{-n}=a m^{n}$, chegamos a uma contradição, pois $m^{n} \notin G$.

Para concluir, mostraremos que se $y \in G$ então $\langle a, y\rangle$ é um grupo abeliano ou é isomorfo a $Q$. Como $a$ não é um elemento central, podemos escolher $g \in G$ tal que $\langle a, g\rangle \cong Q$. Donde 
concluimos que $a$ é um elemento de ordem 4 . Consideremos $A=C_{G}(a)$ e note que $g \notin A$. Temos então para todo $h \in A, h g \notin A, \operatorname{logo}\langle a, h g\rangle \cong Q$. Segue daí que $g^{2}=a^{2}=h g h g$, o qual implica que $g^{-1} h g=h^{-1}$. Notemos também que se $k \notin A$ então $g a g^{-1}=a^{-1}=k a k^{-1}$. Do qual resulta que $g^{-1} k \in A$, e portanto, $A$ é um subgrupo de índice 2 de $G$. Além disso, $A$ é abeliano pois se $h_{1}, h_{2} \in A$ então $g^{-1} h_{1} h_{2} g=\left(h_{1} h_{2}\right)^{-1}$ o que nos mostra que $g^{-1} h_{1} h_{2} g=\left(g^{-1} h_{1} g\right)\left(g^{-1} h_{2} g\right)=$ $h_{1}{ }^{-1} h_{2}{ }^{-2}$, concluindo a demonstração.

$(2) \Rightarrow(1)$ :

Decorre da demonstração do teorema $3.21((2) \Rightarrow(1))$.

Mostramos uma relação entre o 4-centro e segundo centro do grupo de unidades de um anel de grupo inteiro de um grupo periódico.

Proposição 5.17. Seja $G$ um grupo periódico. Então $Z_{2}(\mathcal{U}) \subseteq Z(\mathcal{U}, 4)$.

Demonstração: Sejam $u \in Z_{2}(\mathcal{U})$ e $v \in \mathcal{U}$. Então nós temos que $[u, v] \in Z_{1}(\mathcal{U})$ e $u^{2} \in Z_{2}^{2}(\mathcal{U}) \subseteq$ $Z_{1}(\mathcal{U})$ pelo lema 5.4. Segue-se então que:

$$
[u, v]^{-2}=[u, v]^{-1} u v u^{-1} v^{-1}=u[u, v]^{-1} v u v^{-1}=u^{2} v u^{-2} v^{-1}=1 .
$$

Portanto,

$$
u v u v=u v u^{-1} v^{-1} v u^{2} v=[u, v]^{-1} v^{2} u^{2} \quad \text { pois } \quad u^{2} \in Z_{1}(\mathcal{U}) .
$$

Conseqüentemente,

$$
(u v)^{4}=(u v u v)(u v u v)=\left([u, v]^{-1} v^{2} u^{2}\right)\left([u, v]^{-1} v^{2} u^{2}\right)=[u, v]^{-2} u^{4} v^{4}=u^{4} v^{4} .
$$

O que nos mostra que $u \in Z(\mathcal{U}, 4)$.

Definição 5.18. Seja $\mathbb{Z} G$ o anel de grupo inteiro de um grupo $G$ arbitrário. Um homomorfismo:

$$
f: G \longmapsto \mathcal{U}(\mathbb{Z})=\{ \pm 1\} \quad \text { é dito um homomorfismo de orientação. }
$$

Se $x \in \mathbb{Z} G$. Então definimos $x^{f}$ :

$$
x=\sum_{g \in G} \alpha_{g} g \longmapsto x^{f}=\sum_{g \in G} \alpha_{g} f(g) g^{-1} .
$$

Em particular, se a f é trivial, então $f$ coincide com a involução * usual. 
Lema 5.19. Seja $f: G \longmapsto\{ \pm 1\}$, homomorfismo de orientação, $\operatorname{Ker}(f)=A, f(b)=-1$, onde $G=\langle A, b\rangle, a^{2}=b^{2}, a^{4}=1, b^{-1} a b=a^{-1}$. Ou seja, $G$ é um $Q^{*}$-grupo.

Se u for uma unidade central em $\mathcal{U}(\mathbb{Z} G)$ então $u \in \mathbb{Z} A$.

Demonstração: Qualquer unidade $u \in \mathcal{U}(\mathbb{Z} G)$, pode ser representada na forma $u=x+y b$, onde $x, y \in \mathbb{Z} A$. Suponhamos que $u$ seja uma unidade central em $\mathbb{Z} G$ e que $y \neq 0$.

Pelas proposições 1.40 e 1.39 temos que $\mathbb{Q} G \cong \oplus_{i=1}^{r} M_{n_{i}}\left(D_{i}\right)$; e seja $e_{i}$ o idempotente central associado à i-ésima componente da decomposição de $\mathbb{Q} G$.

Como $u$ é uma unidade central, segue que $u e_{i}=(x+y b) e_{i}$ é central. Denotando por $I d_{n_{i}}$ a matriz identidade de ordem $n_{i}$, concluímos que $(x+y b) e_{i}=\lambda_{i} I d_{n_{i}}$, para algum $\lambda_{i} \in \mathbb{Q}$.

Sejam $x e_{i}=x_{i} I d_{n_{i}}$ e $y e_{i}=y_{i} I d_{n_{i}} \neq(0)$. Nessas condições temos que:

$$
0 \neq(y b) e_{i}=\left(y e_{i}\right)\left(b e_{i}\right)=\left(\lambda_{i}-x_{i}\right) I d_{n_{i}}
$$

donde,

$$
b_{i}=b e_{i}=y_{i}^{-1} I d\left(\lambda_{i}-x_{i}\right) I d, b_{i} \text { central para todo } i, 1 \leq i \leq r, n_{i} \geq 2 .
$$

Como $b_{i}$ e $e_{i}$ são centrais para todo $i$, concluímos que $b$ é central, contradição. Logo, $y=0$ e $u \in \mathbb{Z} A$.

Teorema 5.20. Seja $G$ um grupo periódico. Então são equivalentes:

(1) G é um $Q^{*}$-grupo;

(2) $G$ contém um elemento não-central a tal que $a \in Z(\mathcal{U}, 4)$;

(3) $G$ contém um elemento não-central a tal que $a \in Z(\mathcal{U}, n)$ para algum $n \geq 4$.

Demonstração: $(1) \Rightarrow(2)$ : Se $G$ é um $Q^{*}$-grupo então $G$ possui um subgrupo abeliano $A$ de índice 2 o qual possui um elemento de ordem 4 tal que para todo $h \in A$ e todo $g \in G \backslash A, g^{2}=a^{2}$ e $g^{-1} h g=h^{-1}$. Afirmamos que $a$ não é um elemento central e pertence a $Z_{2}(\mathcal{U})$ e pela proposição 5.17 segue $(2)$.

Seja $f: G \longmapsto\{ \pm 1\}$, homomorfismo de orientação, onde $G=\langle A, b\rangle, \operatorname{Ker}(f)=A, f(b)=$ $-1, b^{2}=a^{2}$. 
Seja $u=a_{1}+a_{2} b, \in \mathcal{U}-1(\mathbb{Z} G), a_{i} \in \mathbb{Z} A, i \in\{1,2\}$ e considere a unidade $u^{f}=a_{1}^{*}-a_{2} a^{2} b$ dada pelo homomorfismo de orientação $f$.

Afirmamos inicialmente que $u^{-1}=u^{f} c^{\prime}$, onde $c^{\prime}$ é uma unidade central. Seja $v=u u^{f}$. Então:

$$
\begin{aligned}
v^{*}=\left(u u^{f}\right)^{*} & =\left(u^{f}\right)^{*}(u)^{*} \\
& =\left(a_{1}-a^{2} b^{*} a_{2}{ }^{*}\right)\left(a_{1}{ }^{*}+b^{*} a_{2}{ }^{*}\right) \\
& =\left(a_{1}-a^{2} b^{-1} a_{2}{ }^{*}\right)\left(a_{1}{ }^{*}+b^{-1} a_{2}{ }^{*}\right) \\
& =a_{1} a_{1}{ }^{*}+a_{1} a_{2} b^{3}-a_{1} a_{2} b-a_{2} a_{2}{ }^{*} \\
& =a_{1} a_{1}{ }^{*}-a_{2} a_{2}{ }^{*}-a_{1} a_{2} b\left(1-b^{2}\right) .
\end{aligned}
$$

Calculando $v v^{*}$ temos que:

$$
\begin{aligned}
v v^{*} & =\left(a_{1} a_{1}{ }^{*}-a_{2} a_{2}{ }^{*}+a_{1} a_{2} b\left(1-b^{2}\right)\right)\left(a_{1} a_{1}{ }^{*}-a_{2} a_{2}{ }^{*}-a_{1} a_{2} b\left(1-b^{2}\right)\right) \\
& =\left(a_{1} a_{1}{ }^{*}\right)^{2}+\left(a_{2} a_{2}{ }^{*}\right)^{2}-2\left(a_{1} a_{1}{ }^{*} a_{2} a_{2}{ }^{*}\right) b^{2} \\
& =\left(a_{1} a_{1}{ }^{*}-a_{2} a_{2}{ }^{*} b^{2}\right)^{2}=c^{2}
\end{aligned}
$$

onde $c=\left(a_{1} a_{1}{ }^{*}-a_{2} a_{2}{ }^{*} b^{2}\right)=c^{*}=c^{f} \in Z_{1}(\mathcal{U})$.

Considere $v_{1}=v c^{-1}, \log \mathrm{O}$ :

$$
v_{1} v_{1}^{*}=v c^{-1}\left(c^{-1}\right)^{*} v^{*}=v v^{*} c^{-1}\left(c^{-1}\right)^{*}=1 .
$$

Pelo lema 1.17 concluímos que $v_{1}= \pm g_{0}$ para algum $g_{0} \in G$ e $v= \pm c g_{0}$.

Consideremos $g_{0}=a_{0} b^{i}, a_{0} \in A, i=0$ ou 1 .

Suponhamos inicialmente que $i=1$. Então obteríamos que $g_{0}=a_{0} b$ e $v= \pm c a_{0} b$, portanto:

$$
\pm c=a_{0}^{-1} v b^{3}=a_{0}^{-1}\left(a_{1} a_{1}{ }^{*}-a_{2} a_{2}{ }^{*}\right)^{2} b^{3}+a_{0}^{-1}\left(a_{1} a_{2}\left(1-b^{2}\right)\right) \in Z_{1}(\mathcal{U}) .
$$

Pelo lema $5.19 c \in \mathbb{Z} A$, logo temos que $a_{0}^{-1}\left(a_{1} a_{1}{ }^{*}-a_{2} a_{2}{ }^{*}\right)^{2} b^{3}=0$. Contradição, pois o aumento de $\left(a_{1} a_{1}{ }^{*}-a_{2} a_{2}{ }^{*}\right)$ é igual a \pm 1 . Logo concluímos que $i=0$ e $g_{0}=a_{0}$. Do qual obtemos que:

$$
a_{0}^{-1}\left(a_{1} a_{1}{ }^{*}-a_{2} a_{2}{ }^{*}\right)+a_{0}^{-1}\left(a_{1} a_{2}\left(1-b^{2}\right) b\right)=a_{0}^{-1} v= \pm c \in \mathbb{Z} A .
$$


Concluímos que $a_{0}^{-1}\left(a_{1} a_{2}\left(1-b^{2}\right) b\right)=0$, logo $\left(a_{1} a_{2}\left(1-b^{2}\right) b\right)=0$. Portanto, $v=u u^{f}=$ $a_{1} a_{1}{ }^{*}-a_{2} a_{2}{ }^{*} \in Z_{1}(\mathcal{U})$. Completando a demonstração.

$(2) \Rightarrow(3)$ : É imediato.

$(3) \Rightarrow(1)$ :

Suponha que $g \in Z(\mathcal{U}, n) \backslash Z_{1}(\mathcal{U})$. Para todo $u \in \mathcal{U}$ e utilizando a proposição 5.13(4) obtemos que:

$$
\left[g, u^{n(1-n)}\right]=[g, u]^{n(1-n)}=\left[g^{n(1-n)}, u\right]=1 .
$$

Portanto, $u^{n(n-1)} g u^{-n(n-1)}=g \in G$ para todo $u \in \mathcal{U}$ e utilizando o teorema 5.16 concluímos que $G$ é um $Q^{*}$-grupo, o que completa a demonstração.

Corolário 5.21. Seja G um grupo periódico. Então as seguintes informações são equivalentes:

(1) G é um $Q^{*}$-grupo;

(2) $G$ contém um elemento não-central a tal que para qualquer unidade $u \in \mathcal{U}, u^{4} a u^{-4}=a$.

Demonstração: $(2) \Rightarrow(1)$ :

É uma conseqüência imediata do teorema 4.

$(1) \Rightarrow(2)$ :

Seja $u \in \mathcal{U}$. Pelo teorema 5.20, $G$ contém um elemento não central $a$ tal que $a \in Z(\mathcal{U}, 4)$. E pela proposição $5.13(3)$ temos que:

$$
\left[a, u^{4}\right]=[a, u]^{4}=\left[a^{4}, u\right]=1 .
$$

Além disso, pelo teorema 5.15 e pela proposição 5.13(5) concluímos que $a^{4} \in Z(\mathcal{U}, 3)=Z_{1}(\mathcal{U})$. Portanto, $u^{4} a u^{-4}=a \in G$ para todo $u \in \mathcal{U}$.

Proposição 5.22. Seja $G$ um grupo periódico e $T_{n}=T(Z(\mathcal{U}, n))=\{x \in Z(\mathcal{U}, n) \mid x$ possui ordem finita e $\varepsilon(x)=1\}$, onde $\varepsilon$ denota o homomorfismo de aumento do anel de grupo inteiro $\mathbb{Z} G$. Então para todo $n \geq 2$,

(1) $T_{n}$ é um subgrupo característico de $Z(\mathcal{U}, n)$. Além disso,

$$
T_{n}=Z(\mathcal{U}, n) \cap G
$$

(2) Se $u \in Z(\mathcal{U}, n)$, então $[u, v] \in T_{n}$ para todo $v \in \mathcal{U}$, 
(3) $Z(\mathcal{U}, n) \subseteq N_{\mathcal{U}}(G)$ e $Z^{2}(\mathcal{U}, n) \subseteq T_{n} Z_{1}(\mathcal{U})$,

(4) $T_{n} \subseteq T\left(Z_{2}(\mathcal{U})\right)$. Além disso, $T_{4}=T\left(Z_{2}(\mathcal{U})\right)$,

(5) $Z(\mathcal{U}, n) \subseteq Z_{2}(\mathcal{U})$. Além disso, $Z(\mathcal{U}, 4)=Z_{2}(\mathcal{U})$.

Demonstração: (1) Pelo lema 1.16 sabemos que as unidades centrais de ordem finita são triviais. Logo, pelo teorema 5.15 é suficiente considerarmos os casos em que $n \geq 4$.

Devemos mostrar que $T_{n}$ é subgrupo de $Z(\mathcal{U}, n)$ para todo $n \geq 4$. Observe inicialmente que se $a \in T_{n}$ então $a^{-1} \in T_{n}$, pois $o\left(a^{-1}\right)=o(a)<\infty$ e $a^{-1} \in Z(\mathcal{U})$, onde $o(a)$ denota a ordem do elemento $a$. Logo é suficiente mostrarmos que se $a, b \in T_{n}$ então o produto $a b \in T_{n}$, ou seja, que $o(a b)<\infty$. Mostraremos isso por indução em $n$.

Sejam $n=4$ e $a, b \in T_{4}$. Suponha que $o(a)=l, o(b)=m$. Portanto,

$(a b)^{4 l m}=\left(a^{4} b^{4}\right)^{l m}=a^{4 l m} b^{4 l m}=1$, pois pelo teorema $5.15, a^{4}, b^{4} \in Z(\mathcal{U}, 3)=Z(\mathcal{U})$.

O que nos mostra que o produto $a b \in T_{4}$ e conseqüentemente $T_{4}$ é um subgrupo.

Suponha agora que para $n=k>3, T_{k}$ seja um subgrupo de $Z(\mathcal{U}, k)$. Devemos mostrar que $T_{k+1}$ é um subgrupo de $Z(\mathcal{U}, k+1)$.

Considere agora $n=k+1$. Sejam $a, b \in T_{k+1} \subseteq Z(\mathcal{U}, k+1)$, então, $(a b)^{k+1}=a^{k+1} b^{k+1}$. Pela proposição 5.13(5), $a^{k+1}$ e $b^{k+1}$ pertencem a $Z(\mathcal{U}, k)$ e ambos possuem ordem finita. Pela hipótese de indução para $T_{k}$, concluímos que $a^{k+1} b^{k+1} \in T_{k}$. Segue que $o\left((a b)^{k+1}\right)=o\left(a^{k+1}\right) o\left(b^{k+1}\right)<\infty$, $\operatorname{logo} o(a b)<\infty$. O que nos mostra que $T_{k+1}$ forma um subgrupo. Portanto, $T_{n}$ é um subgrupo de $Z(\mathcal{U}, n)$ para todo inteiro $n \geq 2$.

Podemos facilmente ver que $\pm T_{n}$ é um subgrupo característico de $Z(\mathcal{U}, n)$. Além disso, como $Z(\mathcal{U}, n)$ é um subgrupo normal do grupo de unidades $\mathcal{U}$ e como $\pm T_{n}$ é um subgrupo característico de $Z(\mathcal{U}, n)$, concluímos que $T_{n}$ é um subgrupo normal do grupo de unidades $\mathcal{U}$. Para observarmos o fato de que $Z(\mathcal{U}, n)$ é um subgrupo normal do grupo de unidades $\mathcal{U}$ perceba que:

Sejam $u \in \mathcal{U}, v \in Z(\mathcal{U}, n)$ segue que $[u, v]^{n}=\left(u^{-1} v^{-1} u v\right)^{n}=\left(u^{-1} v^{-1} u\right)^{n} v^{n}$ ( pois $v \in$ $Z(\mathcal{U}, n))$. O que mostra que $\left(u^{-1} v^{-1} u\right) \in Z(\mathcal{U}, n)$, e portanto $Z(\mathcal{U}, n) \triangleleft \mathcal{U}$.

Segue do teorema 3.2 e do teorema 3.8 , que $T_{n} \triangleleft G$. Portanto, $T_{n}=Z(\mathcal{U}, n) \cap G$.

(2) Sejam $u \in Z(\mathcal{U}, n), v \in \mathcal{U}$. Como $Z(\mathcal{U}, n)$ é um subgrupo normal de $\mathcal{U}$, temos que $v^{-1} u v \in Z(\mathcal{U}, n) ;$ portanto, $[u, v]=u^{-1} v^{-1} u v \in Z(\mathcal{U}, n)$. Além disso,

$$
[u, v]^{n(n-1)}=\left([u, v]^{n(1-n)}\right)^{-1}=1 \text {, pela proposição } 5.13(4) .
$$

Concluímos então que $[u, v] \in T_{n}$. 
(3) Sejam $u \in Z(\mathcal{U}, n), g \in G$. Então por (2) temos que $[u, g] \in T_{n} \subseteq G$ (por (1)). Donde concluímos que $u^{-1} g u \in G$ e conseqüentemente, $Z(\mathcal{U}, n) \subseteq N_{\mathcal{U}}(G)$.

Pelo lema 1.23 temos que:

$$
Z^{2}(\mathcal{U}, n) \subseteq N_{\mathcal{U}}^{2}(G) \subseteq G Z_{1}(\mathcal{U})
$$

Do qual segue facilmente que $Z^{2}(\mathcal{U}, n) \subseteq T_{n} Z_{1}(\mathcal{U})$.

(4) Suponhamos por absurdo que para algum $n \geq 2$, exista um elemento $a \in T_{n}$ tal que $a \notin T\left(Z_{2}(\mathcal{U})\right)$, portanto $a$ é um elemento não central. O teorema 5.20 nos diz que $G$ é um $Q^{*}$-grupo. A seguir mostraremos que esse elemento, é um elemento especial de ordem 4 , como o dado na definição de $Q^{*}$-grupo.

Pela demonstração do teorema 5.16, observamos que se $g \in G$ então:

(a) $\langle a, g\rangle$ é abeliano

$\mathrm{ou}$

(b) $\langle a, g\rangle \cong Q$, o grupo dos quatérnions.

Considerando $A=C_{G}(A) \subseteq G$ e $g \notin A$, nós obtemos que $\langle a, g\rangle \cong Q$, e portanto, $g^{2}=a^{2}$ o que implica que $a$ é um elemento de ordem 4. Para qualquer $h \in A, g \notin A$, temos que $h g \notin A$. Portanto, $\langle a, h g\rangle \cong Q$. Segue daí que $g^{2}=a^{2}=h g h g$, o qual por sua vez implica que $g h g^{-1}=h^{-1}(*)$. Observe também que se $k \notin A$, então repetindo o raciocínio que empregamos anteriormente $g a g^{-1}=a^{-1}=k a k^{-1}$. O que implica que $a g^{-1} k=g^{-1} k a$ e $g^{-1} k \in C_{G}(a)=A$, o que nos mostra que $A$ é um subgrupo de $G$ de índice 2. Pela condição $\left(^{*}\right), A$ é um grupo abeliano; portanto, o elemento $a$ é um elemento especial como afirmamos anteriormente. No entanto, pela demonstração do teorema 5.20, $a \in T\left(Z_{2}(\mathcal{U})\right)$. Contradição. $\operatorname{Logo} T_{n} \subseteq T\left(Z_{2}(\mathcal{U})\right)$. Pela proposição 5.17, segue que $T\left(Z_{2}(\mathcal{U})\right) \subseteq T_{4}$, donde concluímos que $T\left(Z_{2}(\mathcal{U})\right)=T_{4}$.

(5) Sejam $u \in Z(\mathcal{U}, n)$ e $v \in \mathcal{U}$. Então por (2), $[u, v] \in T_{n}$ e por $(4),[u, v] \in T\left(Z_{2}(\mathcal{U})\right)$. Logo $u \in Z_{3}(\mathcal{U})$ e $Z(\mathcal{U}, n) \subseteq Z_{3}(\mathcal{U})$. Como $Z_{3}=Z_{2}$, pelo teorema 5.10, concluimos que $Z(\mathcal{U}, n) \subseteq Z_{2}(\mathcal{U})$. Em particular, temos que $Z(\mathcal{U}, 4) \subseteq Z_{2}(\mathcal{U})$ e pela proposição 5.17 , segue o resultado desejado.

Lema 5.23. Seja $G$ um grupo qualquer e a um elemento genérico de $G$. Se a pertencer a três $n$-centros consecutivos então a é um elemento central de $G$.

Demonstração: Suponha que $a \in(Z(G, n) \cap Z(G, n+1) \cap Z(G, n+2))$. Então para todo $x \in G$ valem as seguintes relações:

$$
(a x)^{n}=a^{n} x^{n},
$$




$$
\begin{aligned}
& (a x)^{n+1}=a^{n+1} x^{n+1}, \\
& (a x)^{n+2}=a^{n+2} x^{n+2} .
\end{aligned}
$$

De 5.12 e 5.13 obtemos:

$$
a^{n+1} x^{n+1}=(a x)^{n+1}=a(x a)^{n} x,
$$

que implica que:

$$
a^{n} x^{n}=(x a)^{n},
$$

e portanto:

$$
(a x)^{n}=(x a)^{n} .
$$

Empregando um raciocínio análogo com 5.13 e 5.14,obtemos:

$$
(a x)^{n+1}=(x a)^{n+1} .
$$

Finalmente de 5.12 e 5.14, obtemos que:

$$
a^{n+2} x^{n+2}=(a x)^{n+2}=a(x a)^{n+1} x=a(x a)^{n}(x a) x \underbrace{=}_{5.15} a(a x)^{n}(x a) x \text {, o que nos permite con- }
$$

cluir que:

$$
a^{n+1} x^{n+1}=(a x)^{n}(x a) .
$$

Substituindo 5.16 em 5.17, obtemos que $a^{n+1} x^{n+1}=(a x)^{n+1}=(a x)^{n}(x a)$.

Do qual resulta imediatamente que:

$$
a x=x a, \text { para todo } x \in G
$$

Finalmente podemos dar a caracterização mencionada ao início da seção:

Teorema 5.24. Seja G um grupo periódico. Então:

$$
Z(\mathcal{U}, n)= \begin{cases}\mathcal{U} & \text { para } n=0 \text { ou } 1 \\ Z_{2}(\mathcal{U}) & \text { para } n=4 k \text { ou } 4 k+1, k \geq 1 \\ Z_{1}(\mathcal{U}) & \text { para } n=4 k+2 \text { ou } 4 k+3, k \geq 0\end{cases}
$$

Demonstração: A primeira igualdade é óbvia.

Para provarmos a segunda igualdade, mostraremos que $Z_{2}(\mathcal{U}) \subseteq Z(\mathcal{U}, 4 k)$ e que $Z_{2}(\mathcal{U}) \subseteq$ $Z(\mathcal{U}, 4 k+1)$ para todo $k \geq 1$. Junto com a proposição $5.22(5)$ isso nos dará o resultado desejado. 
Sejam $u \in Z_{2}(\mathcal{U})$ e $v \in \mathcal{U}$. Então pela proposição 5.17, $u \in Z(\mathcal{U}, 4)$, além disso, pelo teorema 5.15 e pela proposição $5.13(5), u^{4} \in Z_{1}(\mathcal{U})$. Disso segue-se que

$(u v)^{4 k}=\left((u v)^{4}\right)^{k}=\left(u^{4} v^{4}\right)^{k}=u^{4 k} v^{4 k}$.

O que mostra que $u \in Z(\mathcal{U}, 4 k)$, portanto, $Z_{2}(\mathcal{U}) \subseteq Z(\mathcal{U}, 4 k)$.

De maneira totalmente análoga,

$(u v)^{4 k+1}=(u v)(u v)^{4 k}=u v u^{4 k} v^{4 k}=u^{4 k+1} v^{4 k+1}$.

O que significa que $Z_{2}(\mathcal{U}) \subseteq Z(\mathcal{U}, 4 k+1)$.

Suponha agora que $n=4 k+2$ ou $4 k+3, k \geq 1$. Suponhamos inicialmente que $n=4 k+2$. Pela proposição 5.22(5), obtemos que $Z(\mathcal{U}, 4 k+2) \subseteq Z_{2}$, e portanto, $Z(\mathcal{U}, 4 k+2) \subseteq Z(\mathcal{U}, 4 k) \cap$ $Z(\mathcal{U}, 4 k+1)$ pelo que acabamos de ver nos parágrafos anteriores. Se um elemento pertence a três $n$-centros consecutivos, segue pelo lema 5.23 que esse elemento é central.

O caso para $n=4 k+3$ é totalmente análogo ao caso para $n=4 k+2$, de fato, pela proposição $5.22(5)$ temos que $Z(\mathcal{U}, 4 k+3) \subseteq Z_{2}$, mas pelo que acabamos de ver acima, concluímos que $Z(\mathcal{U}, 4 k+3) \subseteq Z(\mathcal{U}, 4 k+1) \cap Z(\mathcal{U}, 4 k+2)$. Novamente se um elemento pertence a três $n$-centros consecutivos ele deve ser central, devido ao lema 5.23. O que completa a demonstração do teorema.

Corolário 5.25. ([3], teorema 3.1) Seja G um grupo finito. Então:

$$
Z(\mathcal{U}, n)= \begin{cases}\mathcal{U} & \text { para } n=0 \text { ou } 1 \\ T\left(Z_{2}(\mathcal{U})\right) Z_{1}(\mathcal{U})=Z_{2}(\mathcal{U}) & \text { para } n=4 k \text { ou } 4 k+1, k \geq 1 \\ Z_{1}(\mathcal{U}) & \text { para } n=4 k+2 \text { ou } 4 k+3, k \geq 0\end{cases}
$$




\section{Apêndice A}

\section{Um resultado de Borel-Chandra}

Nosso objetivo neste apêndice será o de esboçarmos uma idéia não rigorosa da demonstração do teorema de Borel-Chandra, o qual já foi apresentado no capítulo 4 e que enunciaremos mais adiante.

Ao apresentarmos a idéia da demonstração deste resultado, podemos perceber a forte relação entre as diversas áreas da matemática. Em particular, veremos que ele decorre do estudo de propriedades aritméticas de grupos algébricos lineares.

Nos concentraremos nos chamados grupos aritméticos (os quais formam uma classe de subgrupos discretos de grupos algébricos reais). O estudo desses grupos aritméticos surge como conseqüência de uma tentativa de encontrar um tratamento unificado dos diferentes pontos de vista no estudo dos grupos de Lie. Um deles envolvendo subgrupos compactos maximais, espaços simétricos de Riemann (os quais envolvem, Álgebras de Lie, Geometria Diferencial e Topologia) e o outro envolvendo a Geometria Algébrica.

Em ambos os pontos de vista podemos perceber a importância do estudo de domínios fundamentais de grupos aritméticos. Como conseqüência desse estudo, podemos tirar o teorema de Borel-Chandra. (O qual poderia de certo modo ser considerado como conseqüência de um resultado da Geometria!).

Os fatos deste capítulo e informações adicionais estão relacionados nas referências [7], [8], [9], [10], [11], [12], [13], [33].

Nosso objetivo principal neste capítulo será o de apresentarmos uma idéia da demonstração do seguinte:

Teorema A.1. (Borel-Chandra) Sejam $A$ um $\mathbb{Q}$-álgebra de dimensão finita e $O$ uma $\mathbb{Z}$-ordem em A. Então o grupo de unidades de $O$ é finitamente gerado. 
Introduziremos a seguir alguns fatos e definições que serão utilizados no restante do capítulo.

Sejam $k$ um subcorpo de $\mathbb{C}$ e $V$ um $\mathbb{C}$-espaço vetorial de dimensão finita. Diremos que $V$ possui uma $k$-estrutura se ela provêm por extensão dos escalares de um $k$-espaço vetorial $V_{k}$, ou equivalentemente, que nós estamos dando um subespaço vetorial $V_{k}$ sobre $k$ de $V$ tal que $V=V_{k} \bigotimes_{k} \mathbb{C}$

Neste capítulo $V$ designará um espaço vetorial de dimensão finita sobre $\mathbb{C}$, munido de uma $\mathbb{Q}$-estrutura.

Definição A.2. Diremos que um subgrupo $G$ de $G L(n, \mathbb{C})$ é algébrico se ele é o conjunto de todas as matrizes invertíveis cujos coeficientes anulam alguma coleção de polinômios em $n^{2}$ variáveis, com coeficientes complexos. Ele é dito ser definido sobre um subcorpo $k$ de $\mathbb{C}$, se aqueles polinômios puderem ser escolhidos de maneira a possuirem seus coeficientes em $k$. Neste caso diremos que $G$ é um subgrupo algébrico definido sobre $k$ ou que $G$ é um k-grupo.

Se $B$ é um subanel de $\mathbb{C}$ então $G_{B}$ denotará o grupo dos elementos de $G$ que possuem coeficientes em $B$ e cujo determinante é uma unidade de $B$, ou seja, ele é invertível em $B$. Neste caso, $G_{B}=G \cap G L(n, B)$.

Definição A.3. Um subgrupo $G$ de $G L(V)$ é então definido sobre $k$ se existe uma base de $V_{k}$ tal que o isomorfismo correspondente $\beta: G L(V) \longmapsto G L(n, \mathbb{C})$ mapeia $G$ sobre um $k$-grupo (ou grupo algébrico definido sobre $k$ ).

Definição A.4. Sejam $G$ um $\mathbb{Q}$-grupo e $k$ um corpo de números algébricos. Um toro algébrico (ou simplesmente, um toro) é um subgrupo abeliano de $G$ que é diagonalizável sobre $k$ e ambos, $k$-fechado e conexo na topologia de Zariski.

Definição A.5. Uma representação racional $\pi: G \longmapsto G L(V)$ é um homomorfismo de $G$ em $G L(V)$, (onde $V$ é um $\mathbb{C}$-espaço vetorial de dimensão finita), cuja restrição a cada componente conexa de $G$ é uma aplicação racional de $G$ em $\operatorname{End}(V)$, o espaço dos endomorfismos de $V$.

Definição A.6. Se $G$ é um subgrupo algébrico de $G L(V)$ definido sobre $\mathbb{Q}$ e $L$ um lattice de $V_{\mathbb{Q}}$, definimos $G_{L}$, denominado o grupo de $L$-unidades de $G$, o subgrupo de $G_{\mathbb{Q}}$ que estabiliza $L: G_{L}=\left\{g \in G_{\mathbb{Q}} \mid g(L)=L\right\}$.

Definição A.7. Diremos que dois subgrupos $A$ e $B$ de um grupo $H$ são ditos comensuráveis se $A \cap B$ possuir indice finito em $A$ e em $B$.

Exemplo de grupo algébrico definido sobre $\mathbb{Q}$ : 
Seja $G=\operatorname{Tri}(2, \mathbb{C}) \subseteq G L(2, \mathbb{C})$ o conjunto das matrizes triangulares superiores invertíveis com coeficientes complexos. Então claramente $G$ é um subgrupo multiplicativo de $G L(2, \mathbb{C})$. Afirmamos que $G$ é algébrico sobre $\mathbb{Q}$. Consideraremos inicialmente que um elemento genérico de $G L(2, \mathbb{C})$ pode ser expresso na forma: $\left(\begin{array}{ll}x_{1} & x_{2} \\ x_{3} & x_{4}\end{array}\right)$, obedecida a ordem dos elementos na matriz.

Apresentada a caracterização acima de $G L(2, \mathbb{C})$, e considerando o polinômio $q\left(x_{1}, x_{2}, x_{3}, x_{4}\right)=$ $x_{3} \in \mathbb{Q}\left[x_{1}, x_{2}, x_{3}, x_{4}\right]$ temos que qualquer elemento do grupo $G$ satisfaz a condição $q\left(x_{1}, x_{2}, x_{3}, x_{4}\right)=$ $x_{3}=0$. Considerando o polinômio $q\left(x_{1}, x_{2}, x_{3}, x_{4}\right)$ acima, temos que $G$ é um grupo algébrico definido sobre $\mathbb{Q}$.

Definição A.8. Seja $G$ um $\mathbb{Q}$-subgrupo de $G L(V)$. Um subgrupo $\Gamma$ de $G_{\mathbb{Q}}$ é dito ser aritmético se existe um lattice $L$ de $V_{\mathbb{Q}}$ tal que $\Gamma$ seja comensurável $a G_{L}$.

Observamos que se identificarmos $V \operatorname{com} \mathbb{C}^{n}$ por meio de uma base de $L$, então $G$ se identifica a um $\mathbb{Q}$-subgrupo $H$ de $G L(n, \mathbb{C})$ e $G_{L}$ a $H_{\mathbb{Z}}$. Isso equivale a dizer que $\Gamma$ é aritmético se existe uma inclusão $G \subseteq G L(n, \mathbb{C})$ definida sobre $\mathbb{Q}$, que aplique $\Gamma$ sobre um subgrupo de $\rho(G)_{\mathbb{Q}}$ comensurável à $\rho(G)_{\mathbb{Z}}$. Onde $\rho: G \longmapsto G L(n, \mathbb{C})$ denota uma representação racional do grupo $G$.

Nesse sentido, a definição acima pode ser expressa na forma:

Definição A.9. Seja $G$ um $\mathbb{Q}$-grupo algébrico. Um subgrupo $\Gamma$ de $G_{\mathbb{Q}}$ é chamado um grupo aritmético ( ou subgrupo aritmético de $G$ ) se existe uma representação racional fiel $\rho: G \longmapsto$ $G L_{n}$ definida sobre $\mathbb{Q}$ tal que $\rho(\Gamma)$ seja comensurável com $\rho(G) \cap G L(n, \mathbb{Z})$. ( A mesma condição então é verificada para toda $\mathbb{Q}$-representação fiel de $G$ ).

Definição A.10. Um subcorpo $L$ de $\mathbb{C}$ será chamado de corpo de números algébricos se for uma extensão finita de $\mathbb{Q}$ ou equivalentemente, se $L=\mathbb{Q}(\alpha)$, para algum número algébrico $\alpha$; neste caso, todo elemento de $L$ é um número algébrico.

Sejam $S$ um anel e $R$ um subanel de $S$, então denotaremos por $I_{S}(R)$ o conjunto dos elementos de $S$ que são inteiros sobre $R$. Além disso, se $L$ for um corpo de números algébricos, $I_{L}$ denotará o anel de inteiros algébricos de $L$.

Seja $R$ um subanel de um corpo $L$. Nesse caso $Q(R)$ denotará o corpo de frações do anel $R$.

Definição A.11. Seja $M$ um subconjunto multiplicativo do anel R. Definimos o anel de frações de $R$ em relação a $M$, denotado por $R_{M}$. Onde $R_{M}=\left\{\frac{a}{b} \mid a \in R, b \in M\right\}$.

Teorema A.12. ([16], teorema 1.6) Todo dominio fatorial $R$ é integralmente fechado no seu corpo de frações.

Corolário A.13. Se $L=\mathbb{Q}$ temos então que $I_{\mathbb{Q}}=\mathbb{Z}$. 
Teorema A.14. Seja $R$ um subanel do corpo de números algébricos L. Então $Q\left(I_{L}(R)\right)=$ $\left(I_{L}(R)\right)_{R \backslash\{0\}}=I_{L}(Q(R))$. Em particular, $Q\left(I_{L}(R)\right)=L$ se e somente se $L$ é algébrico sobre $Q(R)$.

Demonstração: Seja $\gamma \in Q\left(I_{L}(R)\right)$. Então $\gamma$ pode ser expresso na forma $\frac{\alpha}{\beta}$, onde $\alpha$ e $\beta$ são elementos de $Q\left(I_{L}(R)\right), \beta \neq 0$. Sabemos que $I_{L}(Q(R))$ é um subcorpo de $L$ contendo $I_{L}(R)$ (veja, por exemplo, [16], p.13). Logo temos que $\gamma \in I_{L}(R)$. Para todo $\gamma \in I_{L}(Q(R)$ ), existem elementos $a_{1}, \cdots, a_{m}, d, d \neq 0$ de $R$ tais que $\gamma^{m}+\frac{a_{1}}{d} \gamma^{m-1}+\cdots+\frac{a_{m}}{d}=0$. Multiplicando agora ambos os lados da igualdade por $d^{m}$ obtemos que $\gamma d \in I_{L}(R)$. Logo $\gamma \in\left(I_{L}(R)\right)_{R \backslash\{0\}}$. É imediato que $\left(I_{L}(R)\right)_{R \backslash\{0\}} \subseteq Q\left(I_{L}(R)\right)$. A última afirmação segue ao utilizarmos o fato de que $Q\left(I_{L}(R)\right)=L$ se e somente se $L$ é algébrico sobre $Q(R)$.

Corolário A.15. Seja $L$ um corpo de números algébricos. Então $Q\left(I_{L}\right)=\left(I_{L}\right)_{\mathbb{Z} \backslash\{0\}}=L$. Em particular, se $L=\mathbb{Q}$ temos então que $I_{\mathbb{Q}}=\mathbb{Z}$.

Sejam $k$ um corpo de números de base $\left\{w_{1}, \cdots, w_{d}\right\}$ sobre $\mathbb{Q}$ e $k^{*}$ o grupo multiplicativo do corpo $k$.

Consideremos então a aplicação $T: k^{*} \longmapsto G L(d, \mathbb{C})$, a aplicação definida por $T(\alpha)=T_{\alpha}$, onde $T_{\alpha}$ é definida por $T_{\alpha}(x)=\alpha x$, a representação regular do grupo $k^{*}$.

Podemos considerar, via representação regular, o grupo $k^{*}$ incluído em $G L(d, \mathbb{Q})$. Nessas condições, $T\left(k^{*}\right)$, a imagem de $k^{*}$ em $G L(d, \mathbb{Q})$ se torna o conjunto dos pontos racionais sobre $\mathbb{Q}$ de um grupo $G$, o qual é um toro de dimensão $d$ definido sobre $\mathbb{Q}$. Ou seja, $G$ é diagonalizável e conexo.

De fato, como $\mathbb{Q}$ é um corpo perfeito e a extensão $k \mid \mathbb{Q}$ é algébrica concluímos que ela é uma extensão separável. Logo ela admite um elemento primitivo $\omega \in k$. Por outro lado, sendo uma extensão normal segue que o polinômio $P_{\omega, \mathbb{Q}}$ se fatora num produto de fatores lineares em $k[X]$, onde $P_{\omega, \mathbb{Q}}$ denota o polinômio minimal de $\omega$ sobre $\mathbb{Q}$. O que nos mostra que $G$ é diagonalizável.

Logo, se a base $\left\{w_{i}\right\}$ é formada por inteiros, temos então que $G \cap M_{d}(\mathbb{Z})$ se identifica com o conjunto dos inteiros algébricos não-nulos sobre $k$.

Para observar esse fato, considere a seguir o lattice $\mathcal{L}$ dado por $\mathcal{L}=\oplus_{i=1}^{d} \mathbb{Z} w_{i}$ e observamos que qualquer elemento de $k$ pode ser expresso na forma $\alpha=\sum_{i=1}^{d} \alpha_{i} w_{i}, \alpha_{i} \in \mathbb{Q}$. Não é difícil de observar $\alpha \in k \cap I_{k}$ se e somente se cada $\alpha_{i} \in \mathbb{Z}$.

Conseqüentemente, se a base $\left\{w_{i}\right\}$ é formada por inteiros através do comentário acima podemos concluir neste caso que $G \cap M_{d}(\mathbb{Z})$ se identifica com o conjunto dos inteiros algébricos não-nulos do corpo $k$.

Nessas condições, o grupo aritmético $G_{\mathbb{Z}}$ se identifica com o grupo de unidades do anel de inteiros algébricos do corpo $k$. 
Introduziremos a seguir alguns fatos que serão necessários ao considerarmos o caso mais geral de uma $\mathbb{Q}$-álgebra $A$ de dimensão finita.

Definição A.16. Sejam $A$ e $B$ anéis e $\phi: A \longmapsto B$, homomorfismo injetor de anéis. A aplicação definida por $(a, x) \longmapsto x \phi(a)$ de $A \times B$ em $B$ define em $B$ uma estrutura de $A$-módulo à direita. Em particular, se tomamos $\phi$ a aplicação identidade sobre $A, A$ obtém uma estrutura canônica de A-módulo à direita, a qual denotaremos por $A_{d}$.

Definição A.17. Sejam $A, B$ anéis e $\rho: B \longmapsto A$, um homomorfismo injetor de anéis. Para todo $A$-módulo $E$, a lei externa $(\beta, x) \longmapsto \rho(\beta) x$, define (aditivamente) uma estrutura de $B$-módulo em $E$, a qual denotaremos por $\rho_{*}(E)$.

Definição A.18. Sejam $A, B$ anéis e $\rho: A \longmapsto B$, homomorfismo de anéis. Considere a seguir o $A$-módulo à direita $\rho_{*}\left(B_{d}\right)$ definido por este homomorfismo. Este A-módulo também possui uma estrutura de $B$-módulo à esquerda, a qual será denotada por $B_{s}$ e como $b^{\prime}(b \rho(a))=$ $\left(b^{\prime} b\right) \rho(a)$, para $a \in A, b, b^{\prime} \in B$, estas duas estruturas são compativeis.

Isso nos permite que possamos definir para todo $A$-módulo $E$ à esquerda, uma estrutura de $B$-módulo à esquerda no produto tensorial $\rho_{*}\left(B_{d}\right) \otimes_{A} E$ tal que $\beta^{\prime}(\beta \otimes x)=\left(\beta^{\prime} \beta\right) \otimes x$, para $\beta, \beta^{\prime} \in B$ e $x \in E$.

Este B-módulo à esquerda é dito ser o derivado de $E$ por extensão do anel de escalares de $B$ por meio de $\rho$ e será denotado por $\rho^{*}(E)$ ou $E_{(B)}$.

Proposição A.19. ([13],II.5.1, proposição 4) Seja E um A-módulo admitindo uma A-base $\left(a_{\lambda}\right)_{\lambda \in L}$. Se $\phi: x \longmapsto 1 \otimes x$ é a aplicação canônica de $E$ em $\rho^{*}(E)$, então $\left(\phi\left(a_{\lambda}\right)\right)_{\lambda \in L}$ é uma base de $\rho^{*}(E)$. Se $\rho$ é injetiva então $\phi$ é injetiva.

Proposição A.20. Sejam $A$ um anel, $D$ um corpo e $\rho: A \longmapsto D$, homomorfismo de anéis. Então para qualquer $A$-módulo livre $E$, duas bases quaisquer de $E$ são equipotentes.

Demonstração: Considere o espaço vetorial $E_{(D)}=\rho^{*}(E)=D \otimes_{A} E$ sobre $D$ obtido por extensão do anel de escalares para $D$ e seja $\phi: x \longmapsto 1 \otimes x$ a aplicação canônica de $E$ em $\rho^{*}(E)$; se $\left(a_{\lambda}\right)_{\lambda \in L}$ é uma base de $E$ então pela proposição A.19 temos que $\left(\phi\left(a_{\lambda}\right)\right)_{\lambda \in L}$ é uma base de $\rho^{*}(E)$. Utilizando o fato de que duas bases do mesmo espaço vetorial sobre um corpo são equipotentes a tese segue.

Proposição A.21. Seja $K$ um corpo e $L$ uma extensão de corpos de $K$. Então para todo espaço vetorial $E$ sobre $K$, $\operatorname{dim}_{L}\left(E_{(L)}\right)=\operatorname{dim}_{K}(E)$, onde $E_{(L)}=L \otimes_{K} E$.

Demonstração: De fato, se $\left(a_{\iota}\right)_{\iota \in I}$ é uma base de $E$ sobre $K$, pela proposição A.19 segue que $\left(1 \otimes a_{\iota}\right)_{\iota \in I}$ é uma base de $E_{(L)}$. 
Consideraremos agora o caso mais geral de uma $\mathbb{Q}$-álgebra $A$ de dimensão finita.

Logo $A$ possui uma base $\left\{v_{1}, \cdots, v_{d}\right\}$ sobre $\mathbb{Q}$. Tomando $k=\mathbb{Q}$ e $L=\mathbb{C}$ na proposição A.21 podemos estender os escalares da $\mathbb{Q}$-álgebra $A$. Conseqüentemente designaremos por $A_{\mathbb{C}}$, a álgebra A (vista como espaço vetorial sobre $\mathbb{C}$ ) munida de uma $\mathbb{Q}$-estrutura, a qual é obtida por extensão dos escalares da $\mathbb{Q}$-álgebra $A$, ou seja, $A_{\mathbb{C}}$ pode ser expressa na forma $A_{\mathbb{C}}=A_{\mathbb{Q}} \otimes_{\mathbb{Q}} \mathbb{C}, A_{\mathbb{Q}}$ denotará a álgebra $A$, vista como $\mathbb{Q}$-espaço vetorial.

Considere a seguir o grupo $G$ dos elementos invertíveis de $A_{\mathbb{C}}$, o qual é um grupo algébrico definido sobre $\mathbb{Q}$.

A seguir, sejam $O \subseteq A$, uma $\mathbb{Z}$-ordem em $A_{\mathbb{Q}}$ e $\left\{w_{1}, \cdots, w_{d}\right\}$ uma $\mathbb{Z}$-base de $O$. Considere o lattice $L$ de $A_{\mathbb{Q}}$ dado por $L=\oplus_{i=1}^{d} \mathbb{Z} w_{i}$.

Seja $G_{L}$ o grupo aritmético das unidades do lattice $L$ de $A_{\mathbb{Q}}: G_{L}=\{g \in A \mid g L=L\}$.

Identificando a base $\left\{v_{i}\right\}$ com $\mathbb{C}^{d}$ através de uma base do lattice $L$, temos então que $G$ se identifica a um $\mathbb{Q}$-subgrupo $H$ de $G L(d, \mathbb{C})$ e $G_{L}$ a $H_{\mathbb{Z}}$.

Com as considerações feitas, observamos que o problema de mostrar que o grupo de unidades de uma $\mathbb{Z}$-ordem $O$ de uma $\mathbb{Q}$-álgebra finita $A$ é finitamente gerado se reduz a mostrarmos que o grupo de unidades $G_{\mathbb{Z}}$ de um $\mathbb{Q}$-grupo $G$ é finitamente gerado.

Teorema A.22. (Borel-Chandra) Seja $G \subseteq G L(n, \mathbb{C})$ um grupo algébrico definido sobre $\mathbb{Q}$. Existe um conjunto aberto $U$ em $G_{\mathbb{R}}$ tal que $U \cdot G_{\mathbb{Z}}=G_{\mathbb{R}}$, e que para quaisquer $x, y \in G_{\mathbb{Q}}$, a intersecção $U^{-1} \cdot U \cap x \cdot G_{\mathbb{Z}} \cdot y$ é finita. O grupo $G_{\mathbb{Z}}$ é finitamente gerado.

Teorema A.23. (Borel-Chandra) Sejam A uma $\mathbb{Q}$-álgebra de dimensão finita e $O$ uma $\mathbb{Z}$-ordem em $A$. Então o grupo de unidades de $O$ é finitamente gerado.

Demonstração: Segue pelas considerações acima e observando pelo teorema A.22 que $G_{\mathbb{Z}}$ é finitamente gerado.

O leitor atento poderá constatar que aparentemente poderíamos generalizar a definição de grupo aritmético tomando inicialmente um corpo numérico $k$, um grupo algébrico $G$ definido sobre $k$, uma $k$-representação fiel $\rho: G \longmapsto G L_{n}$ e tomando como grupo aritmético um subgrupo de $G_{k}$ comensurável com $G_{\nu_{k}}=G L\left(n, \nu_{k}\right) \cap \rho(G)$, onde $\nu_{k}$ designa o anel de inteiros de $k$.

No entanto, a classe de grupos aritméticos acima é a mesma que foi descrita anteriormente quando tomamos $k=\mathbb{Q}$. Na realidade utilizando o functor de "restrição de escalares" (uma descrição deste functor e a sua construção pode ser encontrada em [33], cap.1), observamos que se $G^{\prime}=R_{k \mid \mathbb{Q}} G$ é obtido a partir de $G$ por "restrição dos escalares" do corpo $k$ a $\mathbb{Q}$ e utilizando uma base de $\nu_{k}$ sobre $\mathbb{Z}$, temos que $G_{\mathbb{Z}}^{\prime} \cong G_{\nu_{k}}$. 
Considere a seguir $k$ um corpo de números e $B$ o anel de inteiros algébricos do corpo $k$. Então utilizando o functor de "restrição dos escalares" e utilizando os comentários feito no parágrafo anterior, observamos que $G_{B}$ se torna o grupo de unidades do anel de inteiros algébricos do corpo $k$. Esses fatos nos permitem reescrever o teorema de Borel-Chandra na seguinte forma mais geral.

Teorema A.24. (Borel-Chandra) Sejam A uma k-álgebra de dimensão finita e $O$ uma $B$-ordem em $A$, onde $B$ denota o anel de inteiros algébricos do corpo $k$. Então o grupo de unidades de $O$ é finitamente gerado.

Demonstração: Utilizando o functor de "restrição dos escalares" reduzimos o caso descrito no teorema ao caso descrito anteriormente tomando $k=\mathbb{Q}$. E o resultado segue do teorema de Borel-Chandra com $k=\mathbb{Q}$. 


\section{Referências Bibliográficas}

[1] Aleev, R. Z., Higman's Central Unit Theory, Units of Integral Group Rings of Finite Cyclic Groups and Fibonacci Numbers, International Journal of Algebra and Computation 4(1993), number 3, 309-358;

[2] Arora, Satya R., Hales, A.W. and Passi, I.B., Jordan decomposition and hypercentral units in integral group rings, Comm. Alg 21(1993), 25-35;

[3] Arora, Satya R. , Passi, I.B., Central height of the unit group of an integral group ring, Comm. Algebra 21(1993), 3673-3683.

[4] Baer, R., Endlichkeitskriterien für Kommutatorgruppen, Math. Ann. 124(1952), 161-177.

[5] Berman, S.D., On the equation $x^{m}=1$ in an integral group ring, Ukrainsk Mathem. Zh., 7(1955), 253-261.

[6] Blackburn, Norman., Finite groups in which the nonnormal subgroups have nontrivial intersection, J.Algebra 3(1966), 30-37,

[7] Borel, Armand., Linear Algebraic Groups, Second Enlarged Edition, Springer-Verlag, 1991.

[8] Borel, Armand., Arithmetic Properties of Linear Algebraic Groups, Proc. Intern. Congress Math. Stockholm, 1962.Uppsala, 1963.

[9] Borel, Armand., Linear Algebraic Groups, Proc. Sympos. Pure Math., vol.IX, AMS - Providence - USA.

[10] Borel, Armand., Reduction Theory for Arithmetic Groups, Proc. Sympos. Pure Math., vol.IX, AMS - Providence - USA.

[11] Borel, Armand., Introduction Aux Groupes Arithmètiques, Paris - Hermann, 1968.

[12] Borel, A., Chandra, H., Arithmetic Subgroups of Algebraic Groups, Annals of Mathematics, 75(3), 1962.

[13] Bourbaki., Algebra I chap. 1 - 3, Springer-Verlag,1989. 
[14] Bovdi,A.A., The periodic normal divisors of the multiplicative group of a group ring I, Sibirsk Mat. Z. 9(1968), 495-498.

[15] Bovdi,A.A., The periodic normal divisors of the multiplicative group of a group ring II, Sibirsk Mat. Z. 11(1970), 492-511.

[16] Endler, Otto., Teoria dos Números Algébricos, Projeto Euclides, IMPA.

[17] Gorenstein, Finite Groups, Chelsea, 1980 , reprinted by American Mathematical Society.

[18] Gruenberg, K.W., The Engel Structure of Linear Groups, J.Algebra 3(1966), 291-303.

[19] Gruenberg, K.W., The Hypercenter of Linear Groups, J.Algebra 8(1968), 34-40.

[20] Jespers, E., Juriaans, S. O., Isomorphisms of Integral Group Rings of Infinite Rings J.Algebra 233(2000), 171-189;

[21] Kaplansky, I., An introduction to differential algebra, second edition, Hermann, 1976;

[22] Kappe, L.C. and Newell, M.L., On the n-centre of a group. Groups St. Andrews 2, 1989, 339352, London Math. Soc. Lecture Note Ser. 160, Cambridge University Press, Cambridge, 1991.

[23] Li, YuanLin, Parmenter, M.M., Sehgal, S.K., On the Normalizer Property for Integral Group Rings, Comm. Algebra 27(1999), 4217-4223.

[24] Li, YuanLin, The hypercenter and the n-centre of the unit group of an integral group ring, Can.J.Math. vol.50(2), 1998, 401-411.

[25] Marciniak, Z.S., Roggenkamp, K.W., The normalizer of a finite group in its integral group ring and Cech cohomology, pre-print, 1999.

[26] Parmenter, M.M., Conjugates of units in integral group rings, Comm. Algebra 23(1995), 5503-5507.

[27] Ritter, Jürgen, Sehgal, S.K., Integral group rings with trivial central units. Proc. Amer. Math. Soc. 108(1990), 327-329;

[28] Robinson, Derek J.S., A course in the theory of groups,second edition, Springer Verlag, 1995.

[29] Samuel, Pierre.,A propos du théorème des unités. Bull. Sci. Math., 90(1966), 89-96;

[30] Sehgal, S.K., Topics in Group Rings, Marcel Dekker, New York and Basel, 1978.

[31] Sehgal, S. K., Units in Integral Group Rings, Longman, 1993. 
[32] Wehrfritz, B.A.F., Infinite Linear Groups, Springer-Verlag, 1973.

[33] Weil, A., Adeles and Algebraic Groups, Notes by M. Demazure \& T. Ono. The Institute for Advanced Study, Princeton, 1961. 